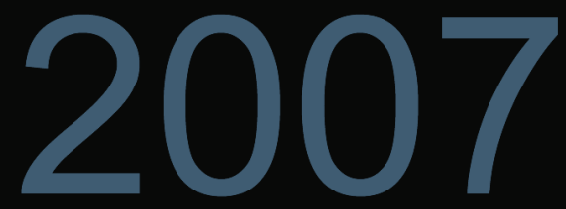

East Tennessee Technology Park Annual Illness and Injury Surveillance Report

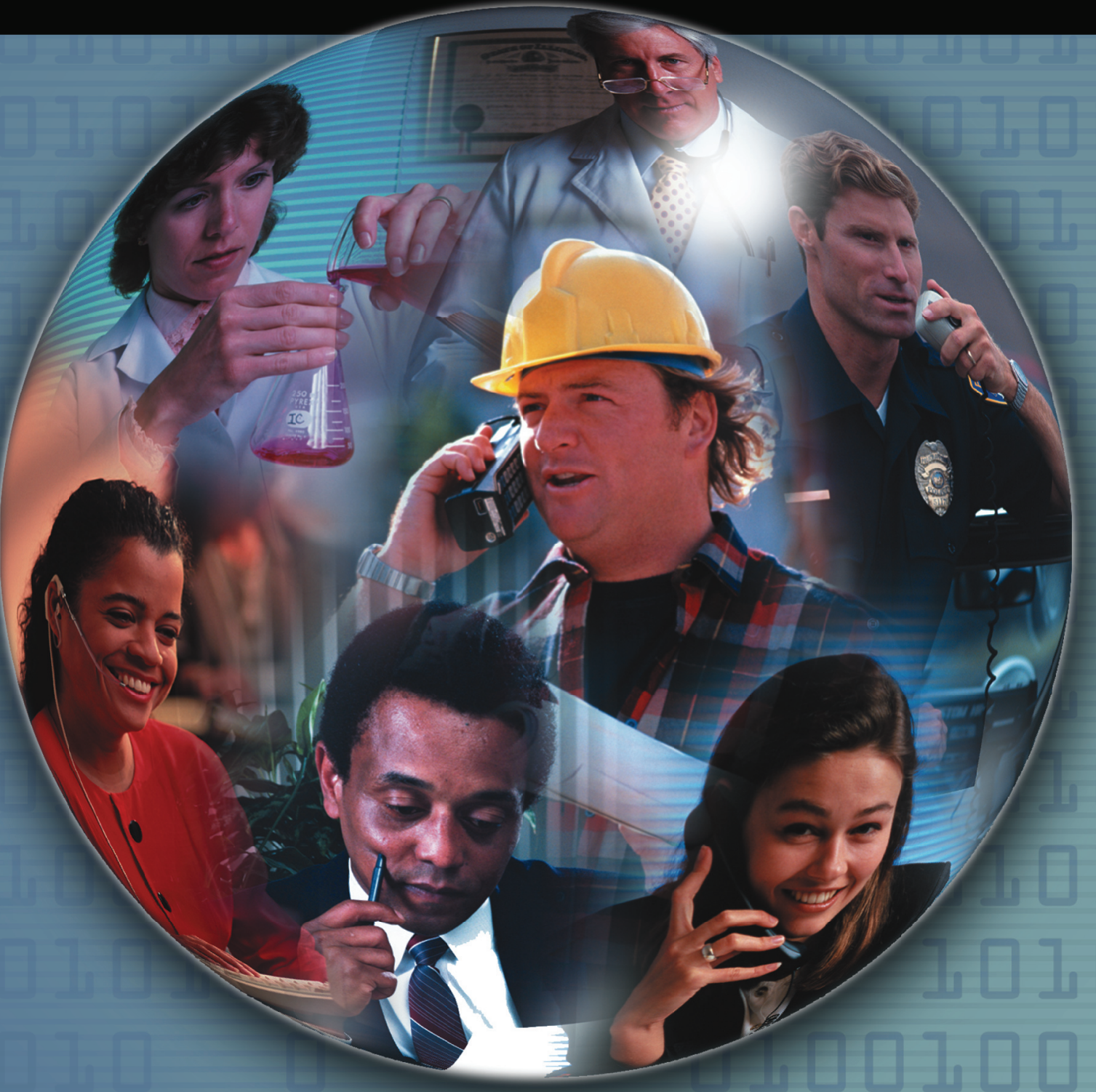




\section{East Tennessee Technology Park 2007 Illness and Injury Surveillance Report}

Questions or comments about this report or the Illness and Injury Surveillance Program (IISP) may be directed to:

E-mail:

Dr. Cliff Strader at cliff.strader@hq.doe.gov or Dr. Bonnie Richter at bonnie.richter@hq.doe.gov

or direct letters to:

Mail Stop HS-13 / 270CC

U.S. Department of Energy

1000 Independence Avenue, S.W.

Washington, DC 20585-0270

Additional information about the Department of Energy's Office of Illness and Injury Prevention Programs, the IISP, and annual reports for DOE sites participating in this program can be found at:

http://www.hss.energy.gov/healthsafety/WSHP/epi/surv/

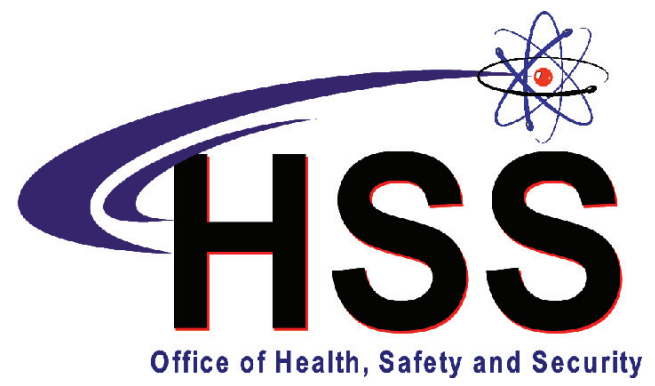

ACKNOWLEDGEMENT

LifeART images copyright 2000 Lippincott Williams \& Wilkins. All rights reserved.

This document was produced under contract number DE-AC05-06OR23100 between the U.S. Department of Energy and Oak Ridge Associated Universities. 


\title{
East Tennessee Technology Park 2007 Illness and Injury Surveillance Report
}

\section{At A Glance}

\begin{abstract}
A total of 1,942 East Tennessee Technology Park (ETTP) employees were included in illness and injury surveillance in 2007. From 2003 to 2007, the work force more than doubled. Since 2003 , the number of men has more than doubled and women have increased almost 50 percent.
\end{abstract}

The average age of workers in 2007 was 46 years for women and 45 years for men. Since 2003, the average age for men has decreased and for women has been unchanged. This decrease in average age of men from 2003 to 2007 is the result of hiring workers less than 30 years of age. In 2003, the percentage of male workers less than 30 years of age was 3 percent; in 2007, the percentage of men in this age group increased to 15 percent. We did not see this shift in age among women.

Women reported 29 absences, resulting in an absence rate of 6.3 absences per 100 workers (29/462). The 1,480 men reported 49 absences, yielding an absence rate of 3.3 per 100 workers $(49 / 1,480)$. The absence rate for both men and women decreased in 2007 after being stable from 2003 to 2006. The higher rate of absences among women compared with men has been generally seen among all DOE sites participating in the Illness and Injury Surveillance Program (IISP).

Technical Support workers had the highest absence rate among men. Among women, the Technical Support and Security and Fire workers had the highest absence rates in 2007. Over the past 5 years, the Technical Support and Security and Fire groups had the highest rates among men. Among women, there has been no apparent trend.

Among the 78 diagnoses reported by men, musculoskeletal conditions and injuries accounted for 54 percent. Among women, 46 percent of the 35 diagnoses were for musculoskeletal conditions and genitourinary disorders.

No definite occupational sentinel health events were identified in 2007. Of the 4 diagnoses possibly resulting from a SHEO, 2 were carpal tunnel syndrome. 
Women reported 6 OSHA-recordable events and men reported 22 OSHA-recordable events in 2007. Twenty-seven of the 29 diagnoses were for injuries, mainly resulting from sprains and strains and open wounds. Four OSHA-recordable events reported by women and 6 OSHArecordable events reported by men were the result of accidents. Half of the accidents were due to overexertion and strenuous movements.

After declining to a minimum of 9.5 events per 1,000 workers in 2004 , the rate of OSHA events increased to 14.4 events per 1,000 workers in 2007 , an increase greater than 50 percent. The overall trend among sites participating in IISP has been a decline in OSHA event rates, but this trend has not been seen at ETTP. The increased rate from 2004 through 2007 is unexplained but could reflect inexperience among newer workers in the work force, which has increased by 43 percent. 


\section{The East Tennessee Technology Park Work Force - 2007}

The Work Force by Gender and Age ........... 1

The Work Force by Gender and Job

Category $\ldots 1$

Number and Length of Absences

Absence Rate by Gender and Age .2

Number of Days Absent by

Gender and Age

Absence Rate by Job Category

and Gender

Average Duration of Absence by

Job Category and Gender.

\section{Diagnostic Categories}

Number of Diagnoses and Lost Calendar

Days by Diagnostic Category

(Categorized by ICD-9-CM) and Gender....... 4

Common Diagnoses Among Female

Workers in 2007

Common Diagnoses Among Male

Workers in 2007

Number of Most Frequently Reported

Diagnoses by Job Category and Gender ......7

\section{Rates of Disease Occurrence}

Rates for All Illnesses and Injuries Combined by Job Category, Gender, and Age

Rates for Selected Diagnostic Categories by Job Category, Gender, and Age. .8

\section{Time Trends}

Age-Adjusted Rates for All Diagnoses Combined Among Women and Men from 2003 to 2007 ...... 10
Age-Adjusted Rates for Selected Diagnostic Categories Among Women and Men from 2003 to 2007

Age-Adjusted Rates for All Diagnoses Combined Among Women and Men by Job Category from 2003 to 2007 ..... 12

\section{Sentinel Health Events for Occupations (SHEOs)}

Characteristics of SHEOs by Gender. 13

SHEO Diagnoses by Gender 13

Occupational Safety and Health Administration (OSHA)-Recordable Events

OSHA-Recordable Events by Gender and Age 14

OSHA-Recordable Events by Job

Category and Gender 14

Diagnostic and Accident Categories for OSHA-Recordable Events

OSHA-Recordable Diagnoses by Diagnostic Category and Gender..... 15

OSHA-Recordable Accidents by Type and Gender.... 16

\section{Rates of OSHA-Recordable Events}

OSHA-Recordable Rates by Age and Job Categories Among Women, All

Diagnoses Combined 17

OSHA-Recordable Rates by Age and Job Categories Among Men, All Diagnoses Combined 17

Time Trends for OSHA-Recordable Events

Age-Adjusted Rates for All OSHA-Recordable Diagnoses Combined Among Women and Men by Job Category from 2003 to $2007 \ldots 18$

\section{Appendices}

Appendices A-W. 20 
The East Tennessee Technology Park Work Force - 2007

Figure 1. The Work Force by Gender and Age

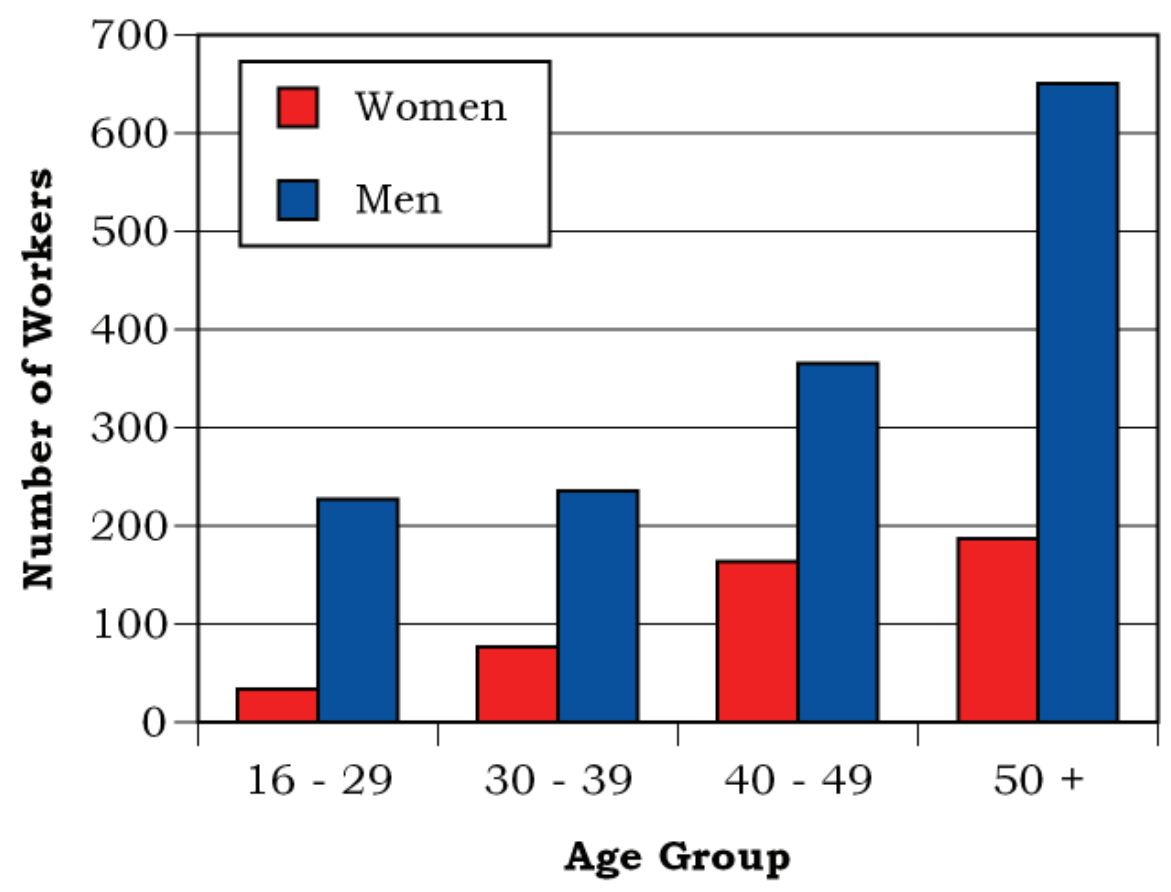

Figure 2. The Work Force by Gender and Job Category

\begin{tabular}{|c|c|c|}
\hline Job Category & Women & Men \\
\hline \multirow{2}{*}{ Professional } & 135 & 539 \\
\hline & $29 \%$ & $36 \%$ \\
\hline \multirow{2}{*}{ Administrative Support } & 146 & 62 \\
\hline & $32 \%$ & $4 \%$ \\
\hline \multirow{2}{*}{ Technical Support } & 8 & 9 \\
\hline & $2 \%$ & $1 \%$ \\
\hline \multirow{2}{*}{ Service } & 150 & 431 \\
\hline & $32 \%$ & $29 \%$ \\
\hline \multirow{2}{*}{ Security and Fire } & 4 & 44 \\
\hline & $1 \%$ & $3 \%$ \\
\hline \multirow{2}{*}{ Crafts } & 10 & 283 \\
\hline & $2 \%$ & $19 \%$ \\
\hline \multirow{2}{*}{ Line Operators } & 9 & 112 \\
\hline & $2 \%$ & $8 \%$ \\
\hline \multirow[t]{2}{*}{ Total } & 462 & 1,480 \\
\hline & $100 \%$ & $100 \%$ \\
\hline
\end{tabular}


Number and Length of Absences

Figure 3. Absence Rate by Gender and Age

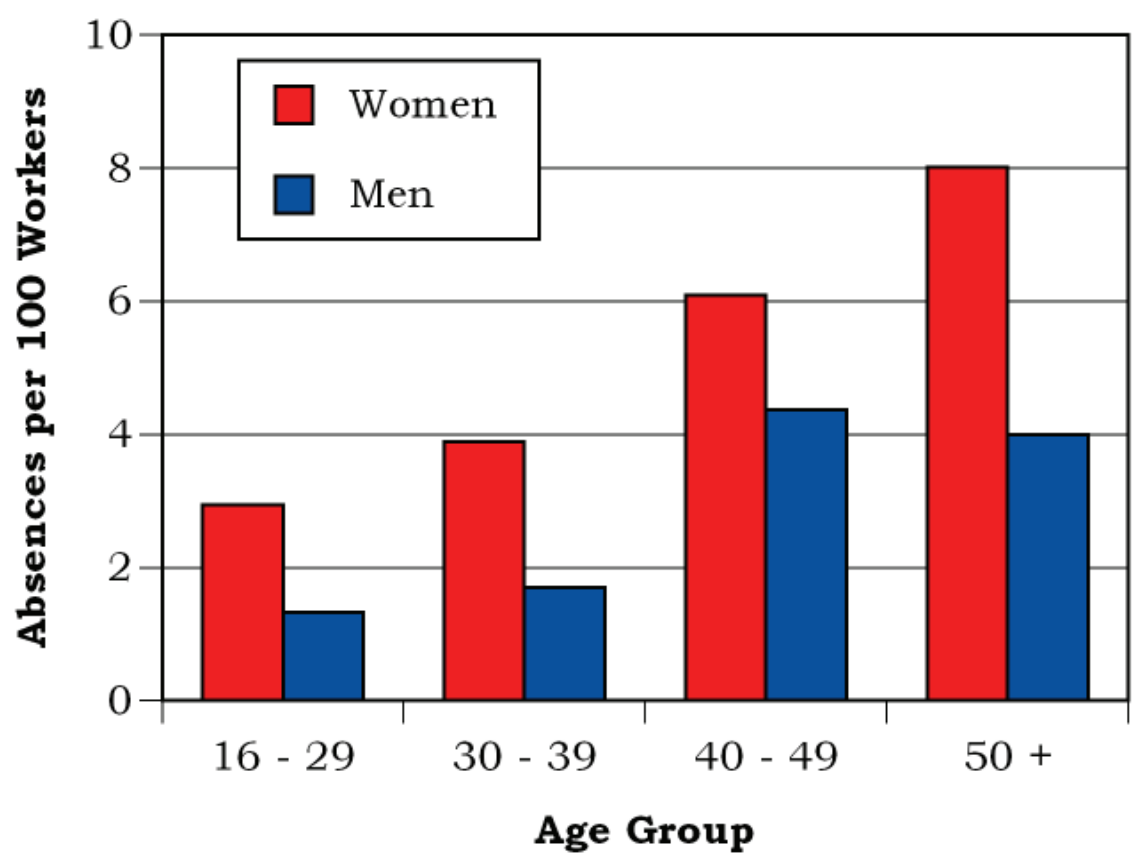

Figure 4. Number of Days Absent by Gender and Age

\begin{tabular}{|c|c|c|c|c|}
\hline \multirow{3}{*}{ Gender } & \multirow{2}{*}{ Age } & \multirow{2}{*}{$\begin{array}{c}\text { Number of } \\
\text { Absences }\end{array}$} & \multicolumn{2}{|c|}{ Number of Days Absent } \\
\cline { 3 - 5 } & & 1 & 18 & 18 \\
\hline \multirow{4}{*}{ Women } & $16-29$ & 1 & 136 & 45 \\
\cline { 2 - 5 } & $30-39$ & 3 & 427 & 43 \\
\cline { 2 - 5 } & $40-49$ & 10 & 558 & 37 \\
\cline { 2 - 5 } & $50+$ & 15 & 1,139 & 39 \\
\cline { 2 - 5 } & Total & 29 & 108 & 36 \\
\hline \multirow{4}{*}{ Men } & $16-29$ & 3 & 212 & 53 \\
\cline { 2 - 5 } & $30-39$ & 4 & 872 & 55 \\
\cline { 2 - 5 } & $40-49$ & 16 & 1,160 & 45 \\
\cline { 2 - 5 } & $50+$ & 26 & 2,352 & 48 \\
\hline
\end{tabular}


Figure 5. Absence Rate by Job Category and Gender

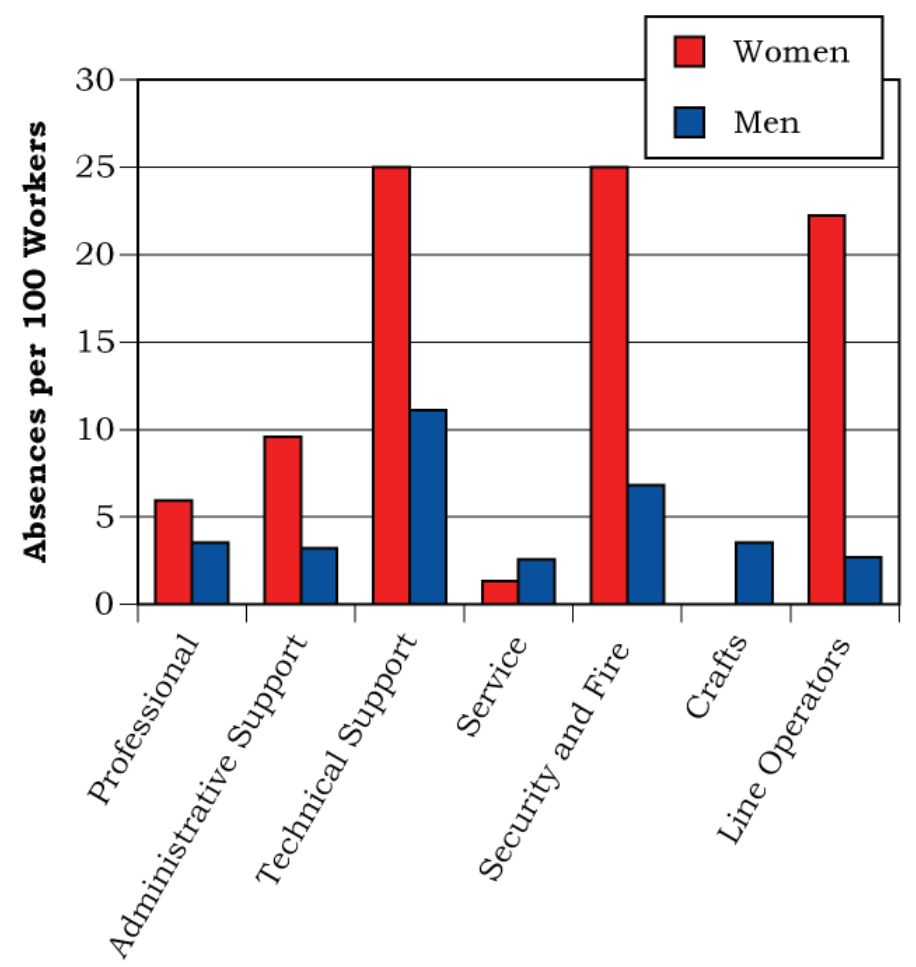

Job Category

Figure 6. Average Duration of Absence by Job Category and Gender

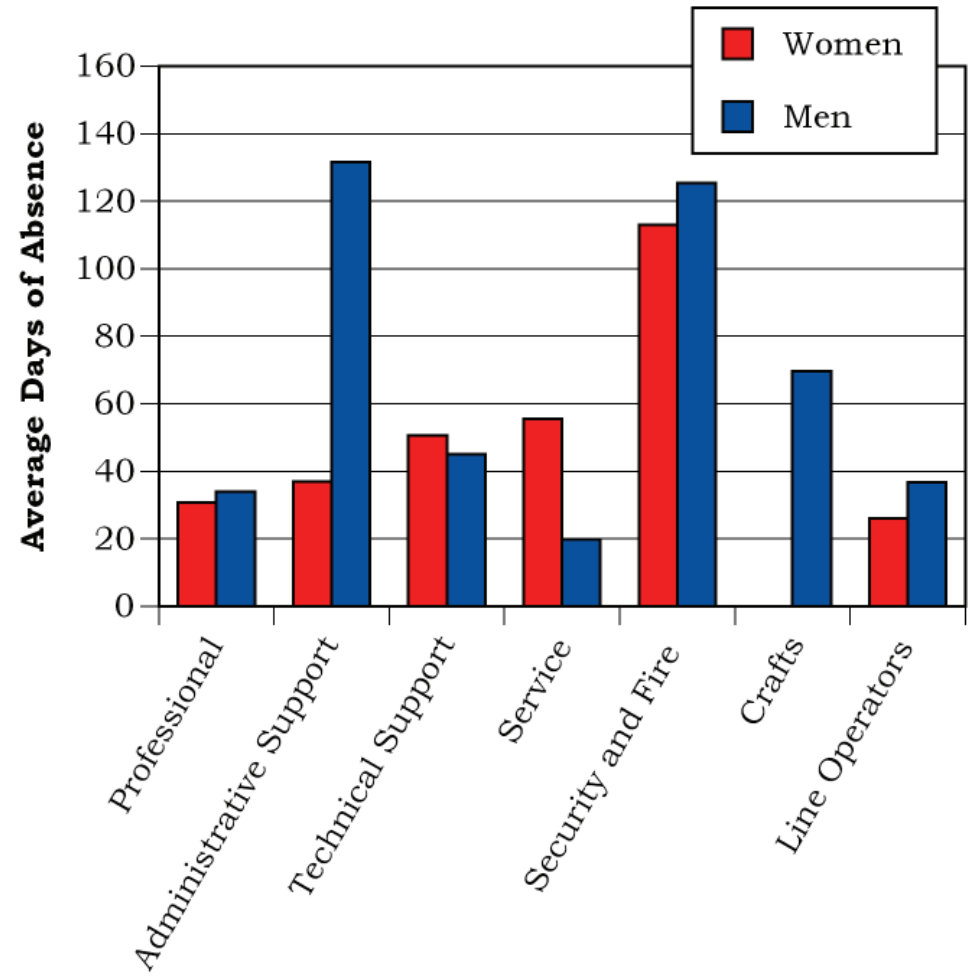

Job Category 


\section{Diagnostic Categories}

\section{Figure 7. Number of Diagnoses and Lost Calendar Days by Diagnostic Category} (Categorized by ICD-9-CM) and Gender

\begin{tabular}{|c|c|c|c|c|}
\hline \multirow[b]{2}{*}{ Diagnostic Category } & \multicolumn{2}{|c|}{ Women } & \multicolumn{2}{|c|}{ Men } \\
\hline & $\begin{array}{l}\text { Number of } \\
\text { Diagnoses }\end{array}$ & $\begin{array}{c}\text { Number } \\
\text { of Lost } \\
\text { Calendar } \\
\text { Days }\end{array}$ & $\begin{array}{l}\text { Number of } \\
\text { Diagnoses }\end{array}$ & $\begin{array}{c}\text { Number } \\
\text { of Lost } \\
\text { Calendar } \\
\text { Days }\end{array}$ \\
\hline Benign Growths & 0 & 0 & 0 & 0 \\
\hline Blood & 0 & 0 & 0 & 0 \\
\hline Cancer & 1 & 68 & 4 & 86 \\
\hline Digestive & 4 & 92 & 6 & 100 \\
\hline Endocrine/Metabolic & 1 & 18 & 1 & 21 \\
\hline Existing Birth Condition & 1 & 130 & 1 & 82 \\
\hline Genitourinary & 8 & 121 & 1 & 19 \\
\hline Heart/Circulatory & 1 & 34 & 5 & 201 \\
\hline Infections / Parasites & 0 & 0 & 0 & 0 \\
\hline Injury & 4 & 192 & 27 & 953 \\
\hline Miscarriage & 0 & 0 & NA & NA \\
\hline Musculoskeletal & 8 & 278 & 15 & 697 \\
\hline Nervous System & 3 & 122 & 5 & 46 \\
\hline Psychological & 0 & 0 & 2 & 10 \\
\hline Respiratory & 3 & 64 & 5 & 76 \\
\hline Skin & 1 & 20 & 0 & 0 \\
\hline Unspecified Symptoms & 0 & 0 & 6 & 278 \\
\hline
\end{tabular}

Note: Lost calendar days for each absence are counted more than once when multiple diagnoses occur in different diagnostic categories for the same absence. 
Figure 8. Common Diagnoses Among Female Workers in 2007

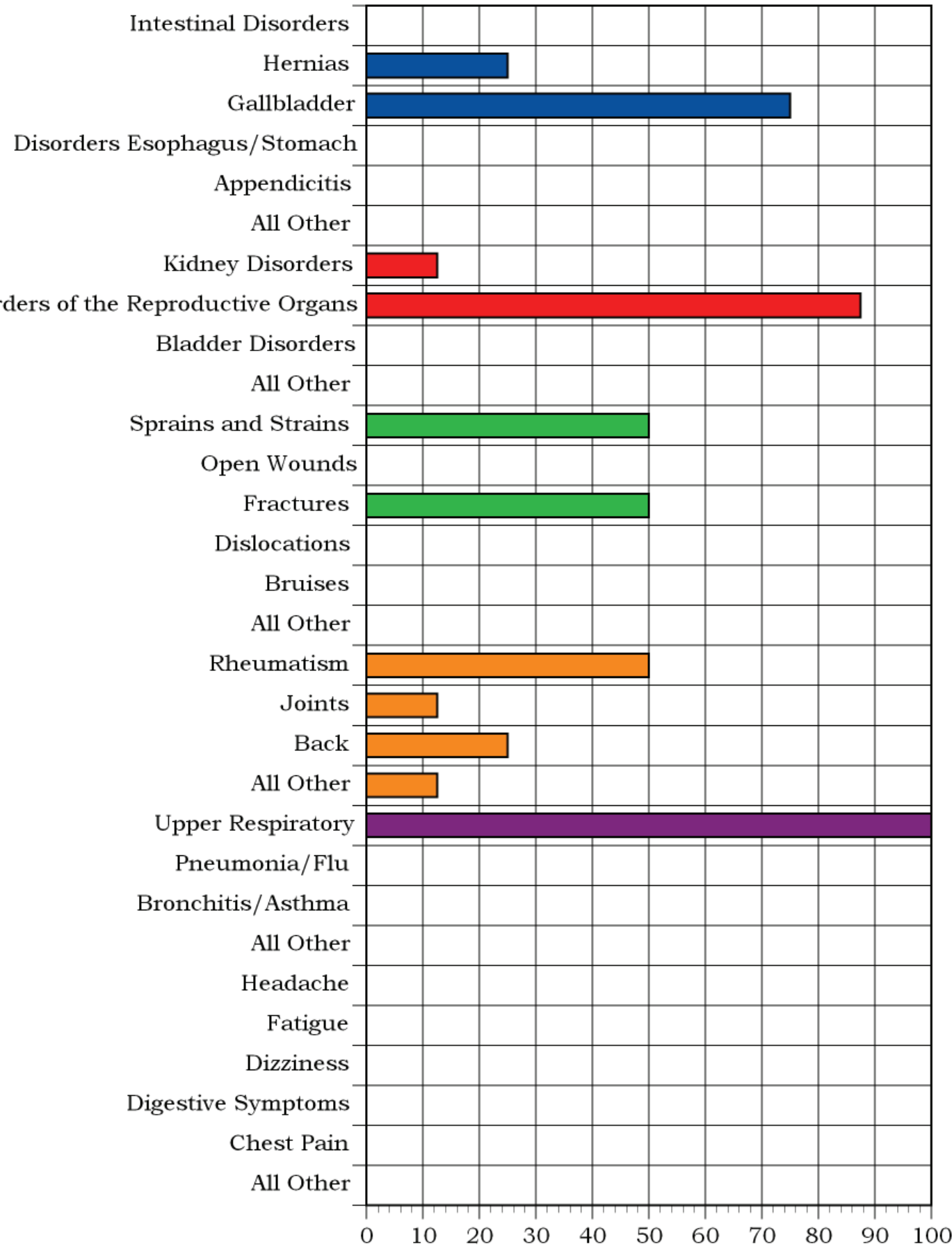

Percent Distribution of Diagnoses Within Diagnostic Category

Digestive, 4 Diagnoses

Genitourinary, 8 Diagnoses

Injury, 4 Diagnosis
Musculoskeletal, 8 Diagnoses

Respiratory, 3 Diagnoses

Unspecified Symptoms, 0 Diagnoses 
Figure 9. Common Diagnoses Among Male Workers in 2007

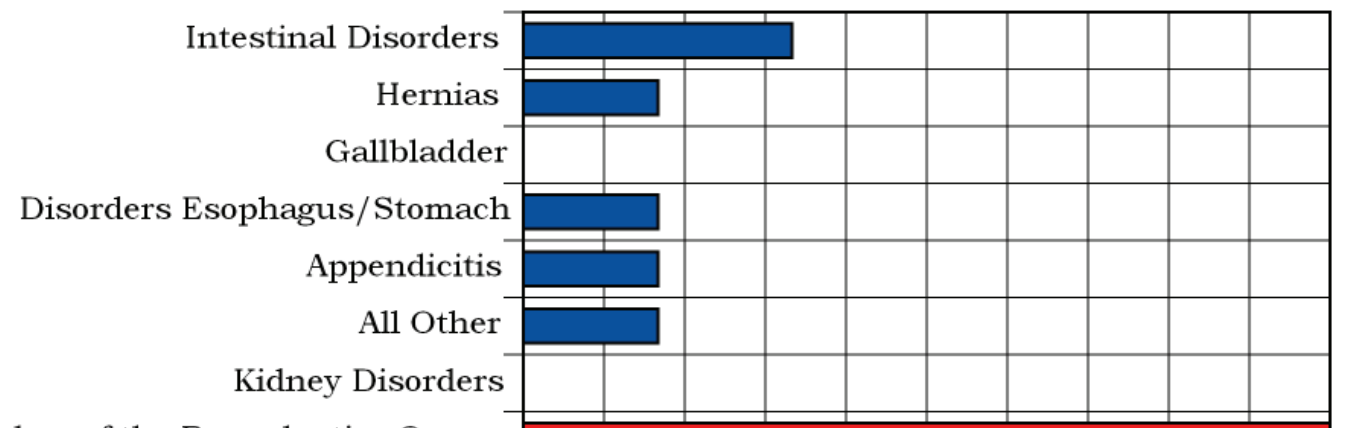

Disorders of the Reproductive Organs
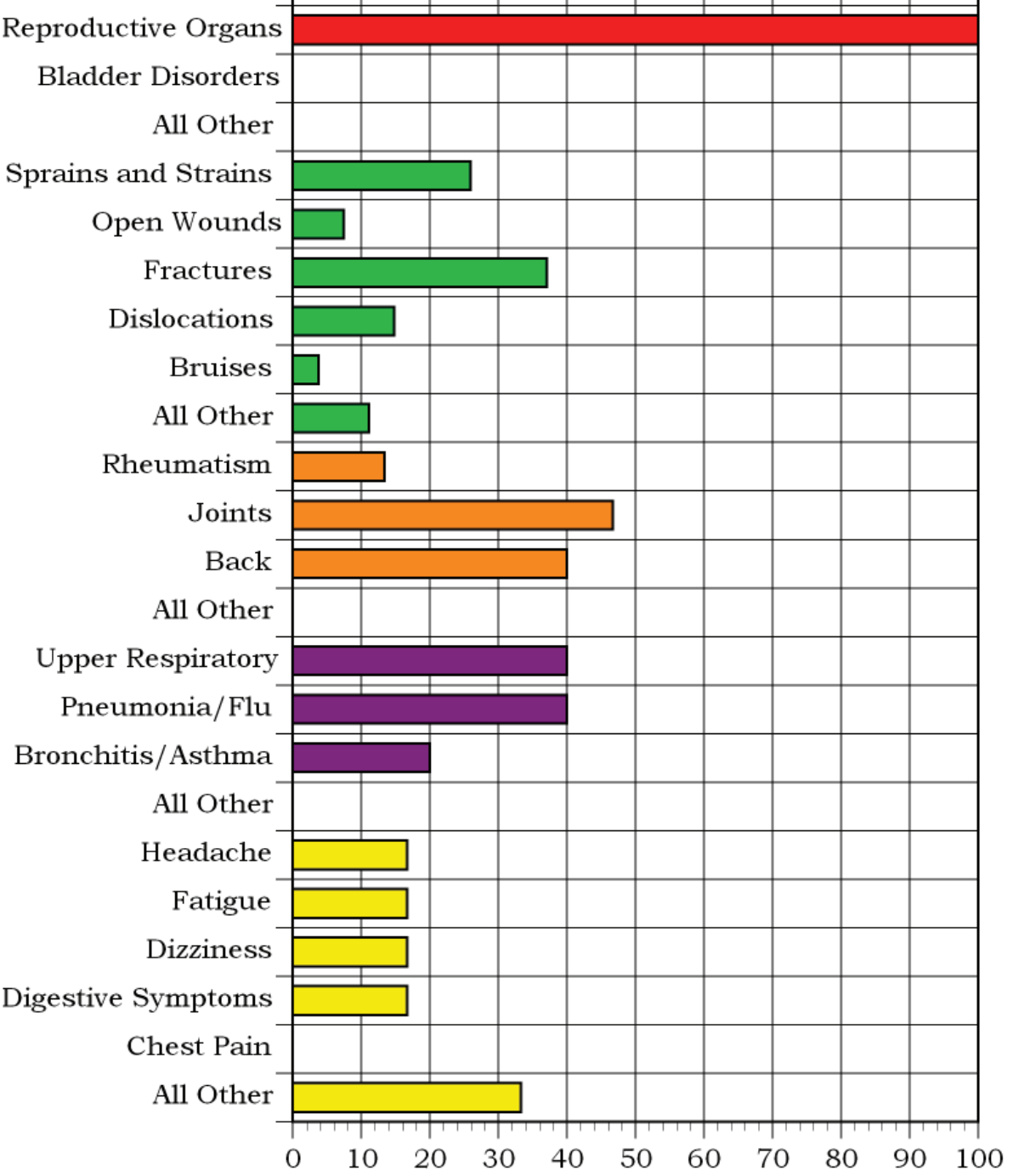

Percent Distribution of Diagnoses Within Diagnostic Category

Digestive, 6 Diagnoses

Genitourinary, 1 Diagnosis

Injury, 27 Diagnoses
Musculoskeletal, 15 Diagnoses

Respiratory, 5 Diagnoses

Unspecified Symptoms, 6 Diagnoses 


\section{Figure 10. Number of Most Frequently Reported Diagnoses by Job Category and Gender}

\begin{tabular}{|c|c|c|c|c|}
\hline Job Category & Men & & Women & \\
\hline \multirow{8}{*}{ Professional } & Injury & 8 & Digestive & 1 \\
\hline & Nervous System & 4 & Endocrine/Metabolic & 1 \\
\hline & Cancer & 2 & Existing Birth Condition & 1 \\
\hline & Heart/Circulatory & 2 & Genitourinary & 1 \\
\hline & Musculoskeletal & 2 & Heart/Circulatory & 1 \\
\hline & Respiratory & 2 & Injury & 1 \\
\hline & Unspecified Symptoms & 2 & Musculoskeletal & 1 \\
\hline & & & Skin & 1 \\
\hline \multirow{4}{*}{ Administrative Support } & Injury & 11 & Genitourinary & 7 \\
\hline & Heart/Circulatory & 1 & Musculoskeletal & 4 \\
\hline & & & Injury & 3 \\
\hline & & & Respiratory & 3 \\
\hline \multirow{2}{*}{ Technical Support } & Digestive & 1 & Musculoskeletal & 1 \\
\hline & & & Nervous System & 1 \\
\hline \multirow{4}{*}{ Service } & Musculoskeletal & 7 & Cancer & 1 \\
\hline & Digestive & 4 & Digestive & 1 \\
\hline & Injury & 3 & & \\
\hline & Respiratory & 3 & & \\
\hline \multirow{3}{*}{ Security and Fire } & Unspecified Symptoms & 3 & Musculoskeletal & 2 \\
\hline & Injury & 1 & & \\
\hline & Musculoskeletal & 1 & & \\
\hline \multirow{4}{*}{ Crafts } & Injury & 4 & & 0 \\
\hline & Musculoskeletal & 4 & & \\
\hline & Heart/Circulatory & 2 & & \\
\hline & Psychological & 2 & & \\
\hline \multirow{3}{*}{ Line Operators } & Cancer & 1 & Nervous System & 2 \\
\hline & Existing Birth Condition & 1 & & \\
\hline & Musculoskeletal & 1 & & \\
\hline
\end{tabular}




\section{Rates of Disease Occurrence}

Figure 11. Rates for All Illnesses and Injuries Combined by Job Category, Gender, and Age

\begin{tabular}{|c|c|c|c|c|}
\hline \multirow{2}{*}{$\begin{array}{c}\text { All Illnesses \& } \\
\text { Injuries Combined }\end{array}$} & \multicolumn{4}{|c|}{$\begin{array}{l}\text { Rate per } 1,000 \\
\end{array}$} \\
\hline & Job Category & Age & Men & Women \\
\hline & \multirow{2}{*}{ Professional } & $<50$ & 71 & 27 \\
\hline & & $50+$ & 32 & 100 \\
\hline & \multirow{2}{*}{ Administrative Support } & $<50$ & 0 & 125 \\
\hline & & $50+$ & 343 & 135 \\
\hline & \multirow{2}{*}{ Technical Support } & $<50$ & 0 & 333 \\
\hline & & $50+$ & 333 & 0 \\
\hline & \multirow{2}{*}{ Service } & $<50$ & 24 & 0 \\
\hline & & $50+$ & 97 & 47 \\
\hline & \multirow{2}{*}{ Security and Fire } & $<50$ & 152 & 500 \\
\hline & & $50+$ & 0 & 0 \\
\hline & \multirow{2}{*}{ Crafts } & $<50$ & 22 & 0 \\
\hline & & $50+$ & 96 & 0 \\
\hline & \multirow{2}{*}{ Line Operators } & $<50$ & 34 & 667 \\
\hline & & $50+$ & 19 & 0 \\
\hline
\end{tabular}

Figure 12. Rates for Selected Diagnostic Categories by Job Category, Gender, and Age

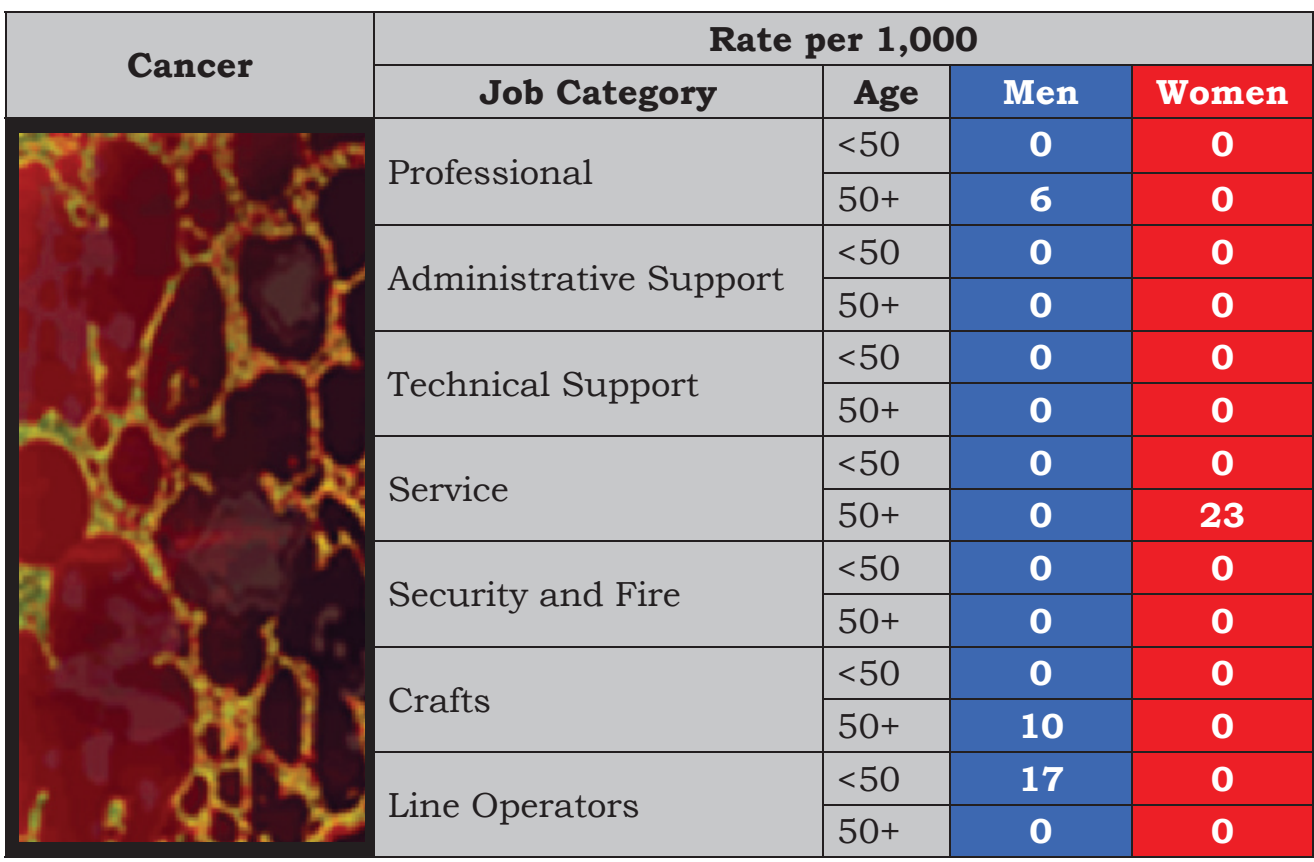




\section{Figure 12. Rates for Selected Diagnostic Categories by Job Category, Gender, and Age (Continued)}

\begin{tabular}{|c|c|c|c|c|}
\hline \multirow{2}{*}{ Heart/Circulatory } & \multicolumn{4}{|c|}{$\begin{array}{r}\text { Rate per } 1,000 \\
\end{array}$} \\
\hline & Job Category & Age & Men & Women \\
\hline & \multirow{2}{*}{ Professional } & $<50$ & 5 & 0 \\
\hline & & $50+$ & 3 & 17 \\
\hline & \multirow{2}{*}{ Administrative Support } & $<50$ & 0 & 0 \\
\hline & & $50+$ & 29 & 0 \\
\hline & \multirow{2}{*}{ Technical Support } & $<50$ & 0 & 0 \\
\hline & & $50+$ & 0 & 0 \\
\hline & \multirow{2}{*}{ Service } & $<50$ & 0 & 0 \\
\hline & & $50+$ & 0 & 0 \\
\hline & \multirow{2}{*}{ Security and Fire } & $<50$ & 0 & 0 \\
\hline & & $50+$ & 0 & 0 \\
\hline & \multirow{2}{*}{ Crafts } & $<50$ & 0 & 0 \\
\hline & & $50+$ & 19 & 0 \\
\hline & \multirow{2}{*}{ Line Operators } & $<50$ & 0 & 0 \\
\hline & & $50+$ & 0 & 0 \\
\hline
\end{tabular}

\begin{tabular}{|c|c|c|c|c|}
\hline \multirow{2}{*}{ Respiratory } & \multicolumn{4}{|c|}{ Rate per 1,000} \\
\hline & Job Category & Age & Men & Women \\
\hline & \multirow{2}{*}{ Professional } & $<50$ & 5 & 0 \\
\hline & & $50+$ & 3 & 0 \\
\hline & \multirow{2}{*}{ Administrative Support } & $<50$ & 0 & 0 \\
\hline & & $50+$ & 0 & 41 \\
\hline & \multirow{2}{*}{ Technical Support } & $<50$ & 0 & 0 \\
\hline & & $50+$ & 0 & 0 \\
\hline & \multirow{2}{*}{ Service } & $<50$ & 0 & 0 \\
\hline & & $50+$ & 29 & 0 \\
\hline & \multirow{2}{*}{ Security and Fire } & $<50$ & 0 & 0 \\
\hline & & $50+$ & 0 & 0 \\
\hline & \multirow{2}{*}{ Crafts } & $<50$ & 0 & 0 \\
\hline & & $50+$ & 0 & 0 \\
\hline & \multirow{2}{*}{ Line Operators } & $<50$ & 0 & 0 \\
\hline & & $50+$ & 0 & 0 \\
\hline
\end{tabular}


Figure 12. Rates for Selected Diagnostic Categories by Job Category, Gender, and Age (Continued)

\begin{tabular}{|c|c|c|c|c|}
\hline \multirow{2}{*}{ Injury } & \multicolumn{4}{|c|}{ Rate per 1,000} \\
\hline & Job Category & Age & Men & Women \\
\hline & \multirow{2}{*}{ Professional } & $<50$ & 36 & 0 \\
\hline & & $50+$ & 3 & 17 \\
\hline & \multirow{2}{*}{ Administrative Support } & $<50$ & 0 & 0 \\
\hline & & $50+$ & 314 & 41 \\
\hline & \multirow{2}{*}{ Technical Support } & $<50$ & 0 & 0 \\
\hline & & $50+$ & 0 & 0 \\
\hline & \multirow{2}{*}{ Service } & $<50$ & 6 & 0 \\
\hline & & $50+$ & 10 & 0 \\
\hline & \multirow{2}{*}{ Security and Fire } & $<50$ & 30 & 0 \\
\hline & & $50+$ & 0 & 0 \\
\hline & \multirow{2}{*}{ Crafts } & $<50$ & 17 & 0 \\
\hline & & $50+$ & 10 & 0 \\
\hline & \multirow{2}{*}{ Line Operators } & $<50$ & 0 & 0 \\
\hline & & $50+$ & 0 & 0 \\
\hline
\end{tabular}

\section{Time Trends}

Figure 13. Age-Adjusted Rates for All Diagnoses Combined Among Women and Men from 2003 to 2007*

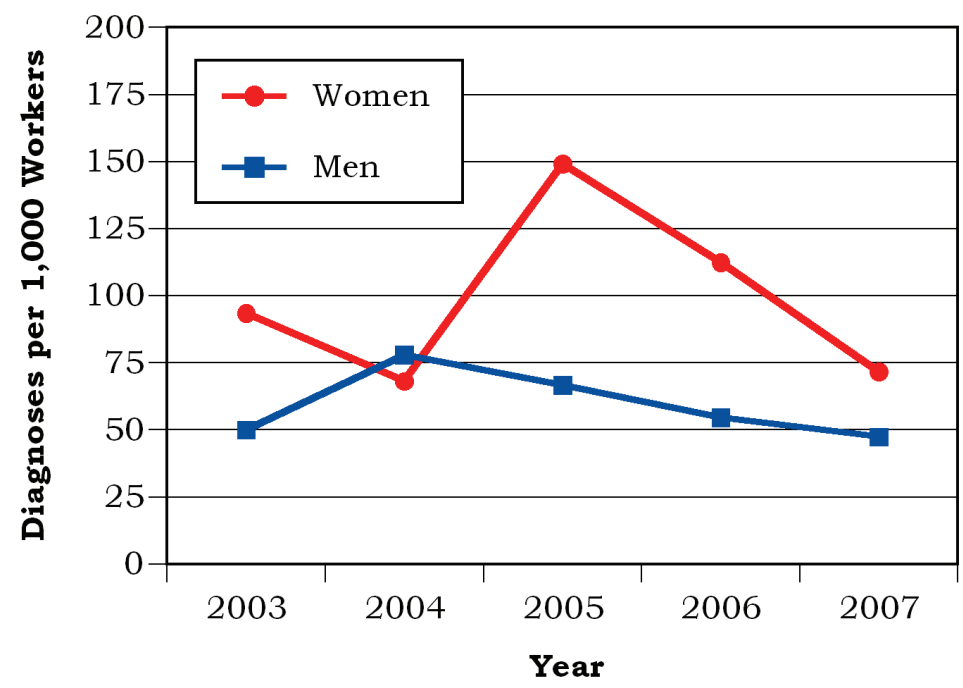

* Standardized to age distribution of 2000 U.S. population. 
Figure 14. Age-Adjusted Rates for Selected Diagnostic Categories Among Women and Men from 2003 to 2007*

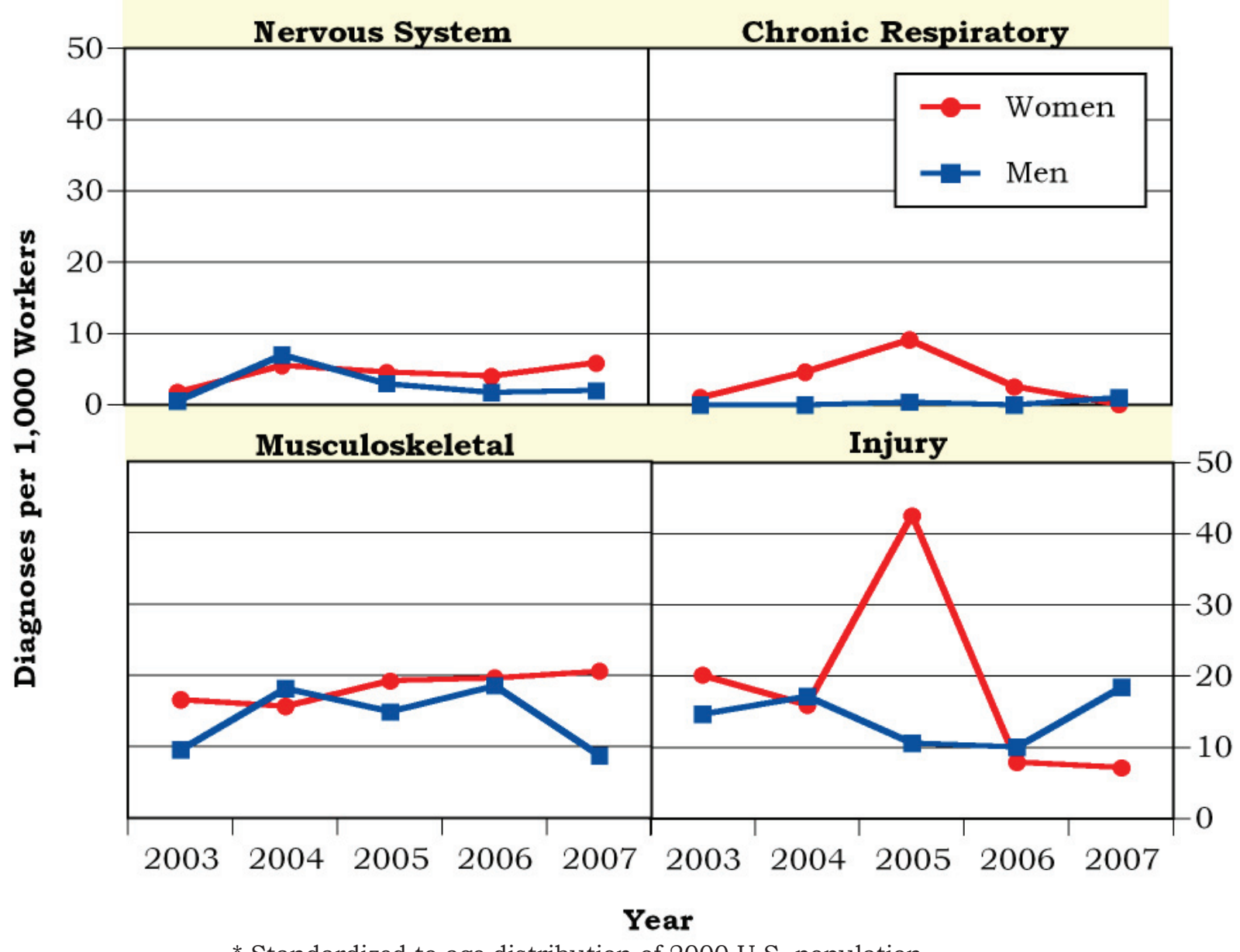

* Standardized to age distribution of 2000 U.S. population. 
Figure 15. Age-Adjusted Rates for All Diagnoses Combined Among Women and Men by Job Category from 2003 to 2007*

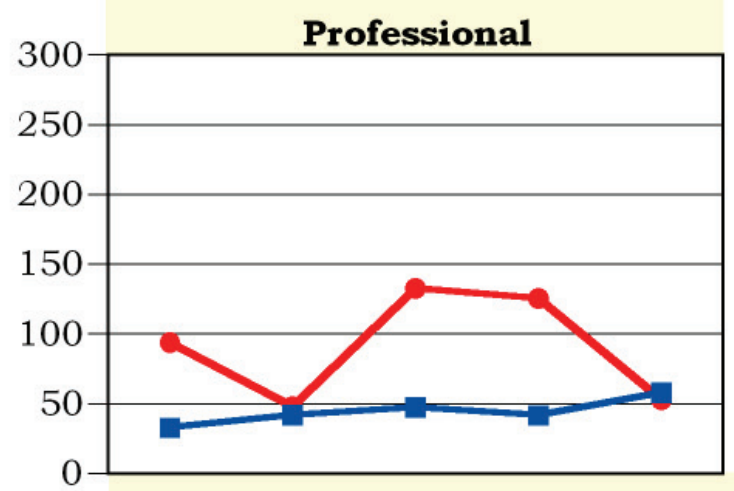

- Women

$\rightarrow$ Men

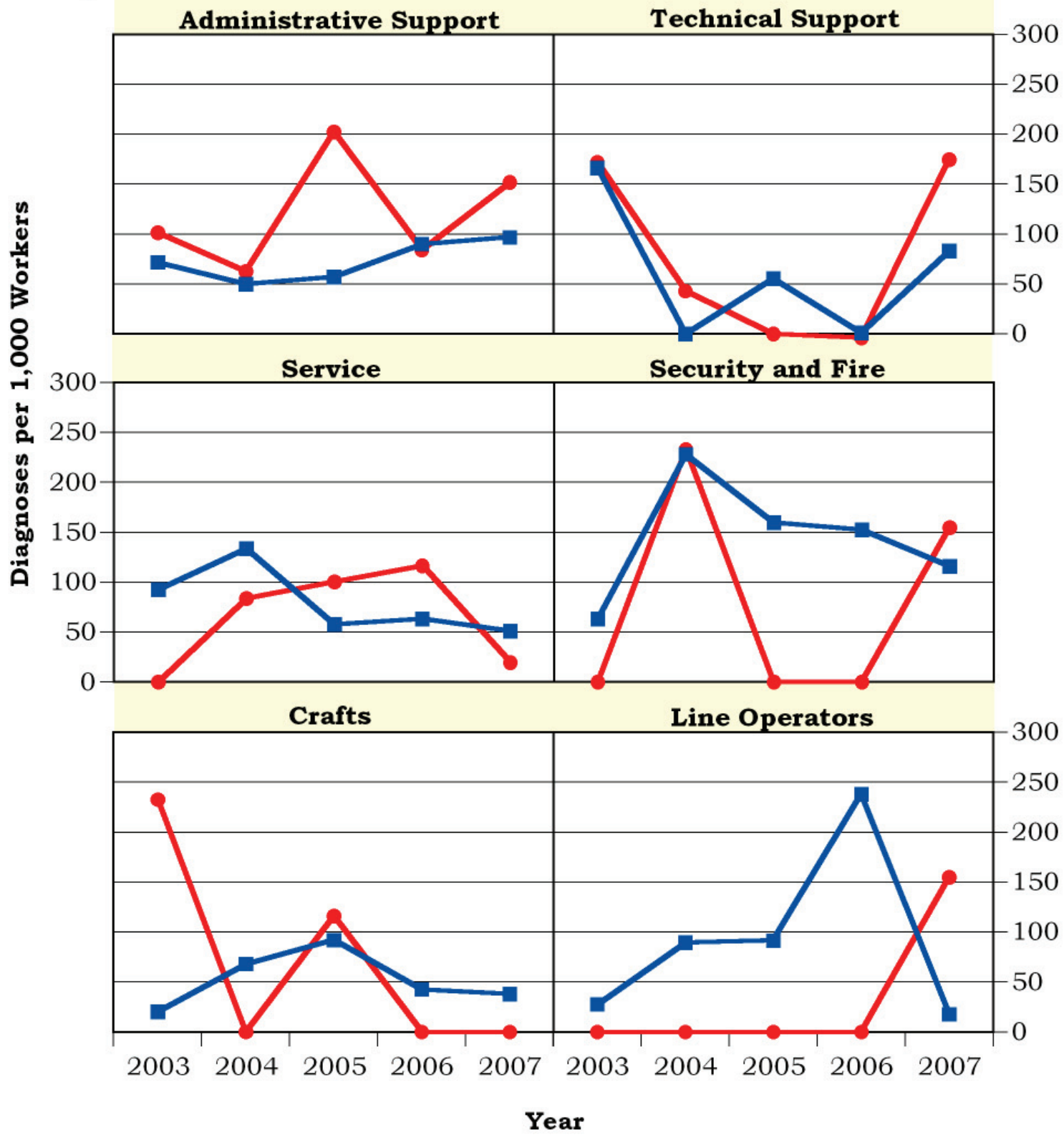

* Standardized to age distribution of 2000 U.S. population. 


\section{Sentinel Health Events for Occupations (SHEOs)}

An occupational sentinel health event (SHEO) is a disease, disability, or death that is likely to be occupationally related. Although sentinel health events may indicate an occupational exposure, many may result from nonoccupational exposures. Sentinel health events are therefore assessed in two categories:

Definite Sentinel Health Events: Diseases that are unlikely to occur in the absence of an occupational exposure (e.g., asbestosis).

Possible Sentinel Health Events: Diseases that may be occupational but can also occur in the absence of an occupational exposure (e.g., lung cancer or carpal tunnel syndrome).

Figure 16. Characteristics of SHEOs by Gender

\begin{tabular}{|l|c|c|c|c|}
\hline \multirow{2}{*}{} & \multicolumn{2}{|c|}{$\begin{array}{c}\text { Total Number of } \\
\text { SHEO Diagnoses }\end{array}$} & \multicolumn{2}{c|}{$\begin{array}{c}\text { Total Number of } \\
\text { Days Absent }\end{array}$} \\
\cline { 2 - 5 } & Men & Women & Men & Women \\
\hline Definite & 0 & 0 & 0 & 0 \\
\hline Possible & 2 & 2 & 79 & 52 \\
\hline Total & 2 & 2 & 79 & 52 \\
\hline
\end{tabular}

Figure 17. SHEO Diagnoses by Gender

\begin{tabular}{|l|c|c|}
\hline \multirow{2}{*}{\multicolumn{1}{|c|}{ Diagnoses }} & \multicolumn{2}{c|}{ Gender } \\
\cline { 2 - 3 } & Women & Men \\
\hline Carpal Tunnel Syndrome & 2 & 0 \\
\hline Pneumonconiosis (Berylliosis) & 0 & 0 \\
\hline Musculoskeletal Conditions & 0 & 0 \\
\hline Injuries & 0 & 0 \\
\hline Other Conditions & 0 & 2 \\
\hline
\end{tabular}


Occupational Safety and Health Administration (OSHA)-Recordable Events

Figure 18. OSHA-Recordable Events by Gender and Age

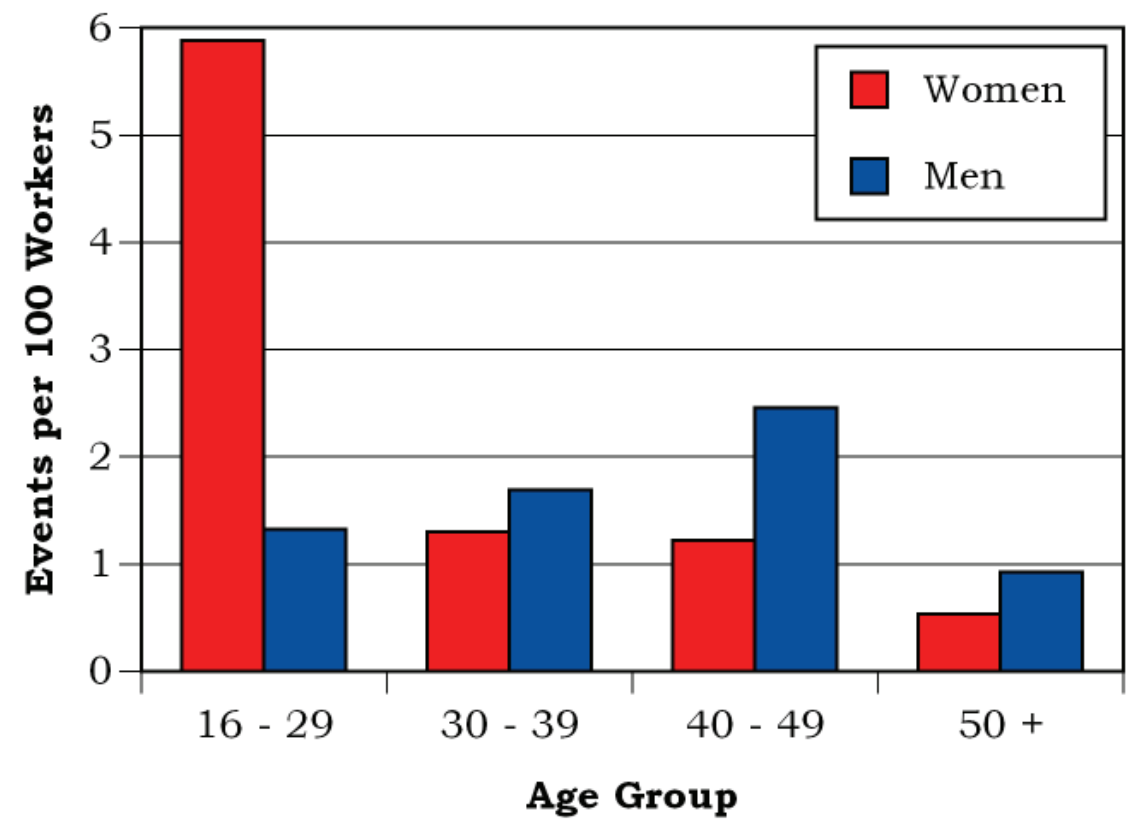

Figure 19. OSHA-Recordable Events by Job Category and Gender

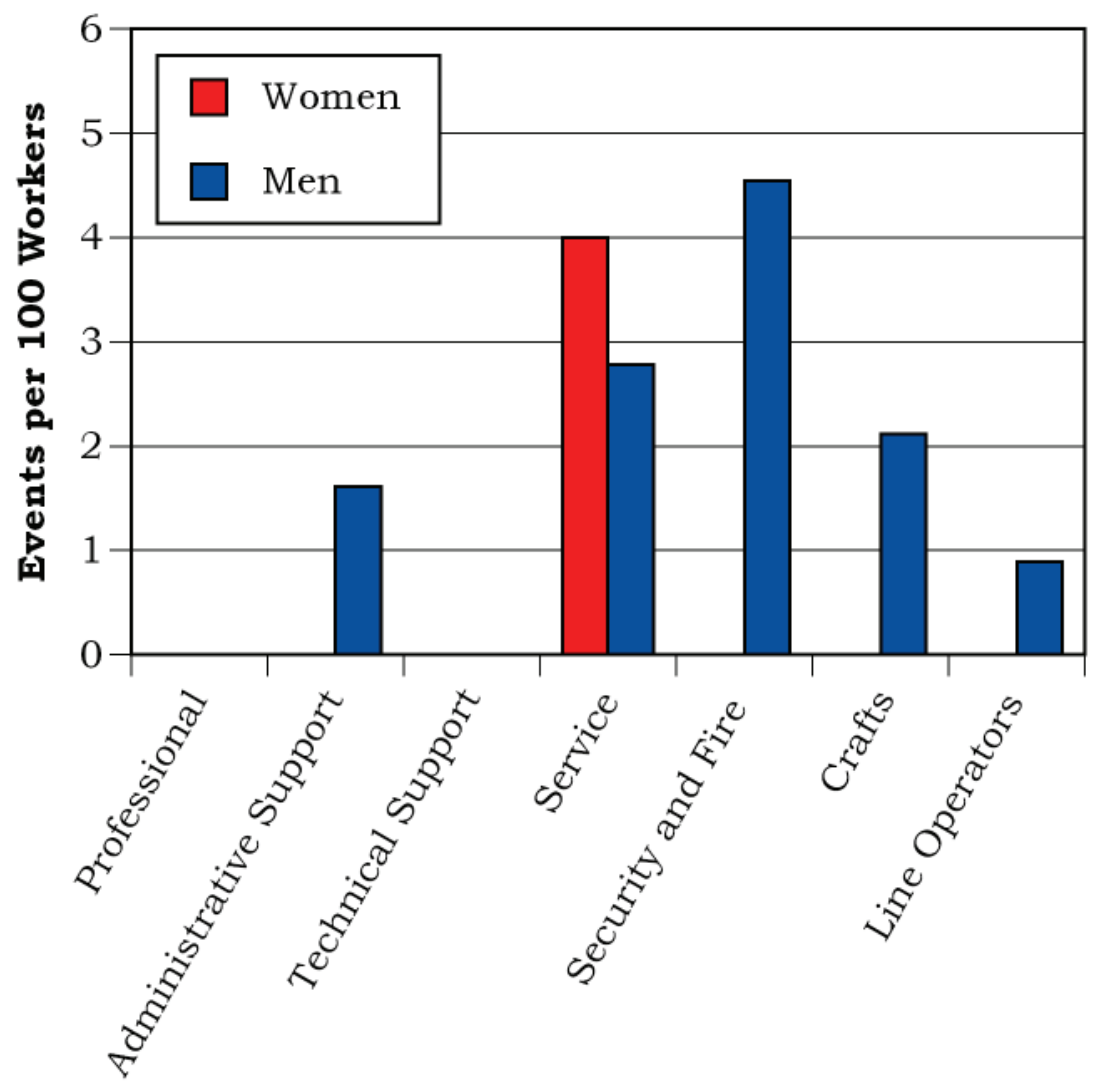

Job Category 


\section{Diagnostic and Accident Categories for OSHA-Recordable Events}

Figure 20. OSHA-Recordable Diagnoses by Diagnostic Category and Gender

\begin{tabular}{|l|c|c|}
\hline \multirow{2}{*}{ Diagnostic Category } & \multicolumn{2}{|c|}{ Gender } \\
\cline { 2 - 3 } & Women & Men \\
\hline Nervous System & $\mathbf{0}$ & $\mathbf{2}$ \\
\hline Injury & $\mathbf{6}$ & $\mathbf{2 1}$ \\
\hline Fractures - Upper Limb & $\mathbf{0}$ & $\mathbf{3}$ \\
\hline Fractures - Lower Limb & $\mathbf{0}$ & $\mathbf{1}$ \\
\hline Back Sprains \& Strains & $\mathbf{0}$ & $\mathbf{3}$ \\
\hline Other Sprains \& Strains & $\mathbf{4}$ & $\mathbf{5}$ \\
\hline Open Wounds - Upper Limb & $\mathbf{1}$ & $\mathbf{4}$ \\
\hline Open Wounds - Lower Limb & $\mathbf{1}$ & $\mathbf{1}$ \\
\hline Bruises & $\mathbf{0}$ & $\mathbf{3}$ \\
\hline Burns & $\mathbf{0}$ & $\mathbf{1}$ \\
\hline
\end{tabular}


Figure 21. OSHA-Recordable Accidents by Type and Gender

\begin{tabular}{|l|c|c|}
\hline \multirow{2}{*}{\multicolumn{2}{|c}{ Accident Category }} & \multicolumn{2}{|c|}{ Gender } \\
\cline { 2 - 3 } & Women & Men \\
\cline { 2 - 3 } & $\begin{array}{c}\text { Number of } \\
\text { Accidents }\end{array}$ & $\begin{array}{c}\text { Number of } \\
\text { Accidents }\end{array}$ \\
\hline Falls & $\mathbf{1}$ & $\mathbf{1}$ \\
\hline Other Accidents & $\mathbf{3}$ & $\mathbf{5}$ \\
\hline Cutting/Piercing Instrument/Object & $\mathbf{2}$ & $\mathbf{0}$ \\
\hline $\begin{array}{l}\text { Hot, Corrosive, or Caustic Material/ } \\
\text { Steam }\end{array}$ & $\mathbf{0}$ & $\mathbf{1}$ \\
\hline Overexertion/Strenuous Movements & $\mathbf{1}$ & $\mathbf{4}$ \\
\hline Total & $\mathbf{4}$ & $\mathbf{6}$ \\
\hline
\end{tabular}




\section{Rates of OSHA-Recordable Events}

Figure 22. OSHA-Recordable Rates by Age and Job Categories Among Women, All Diagnoses Combined

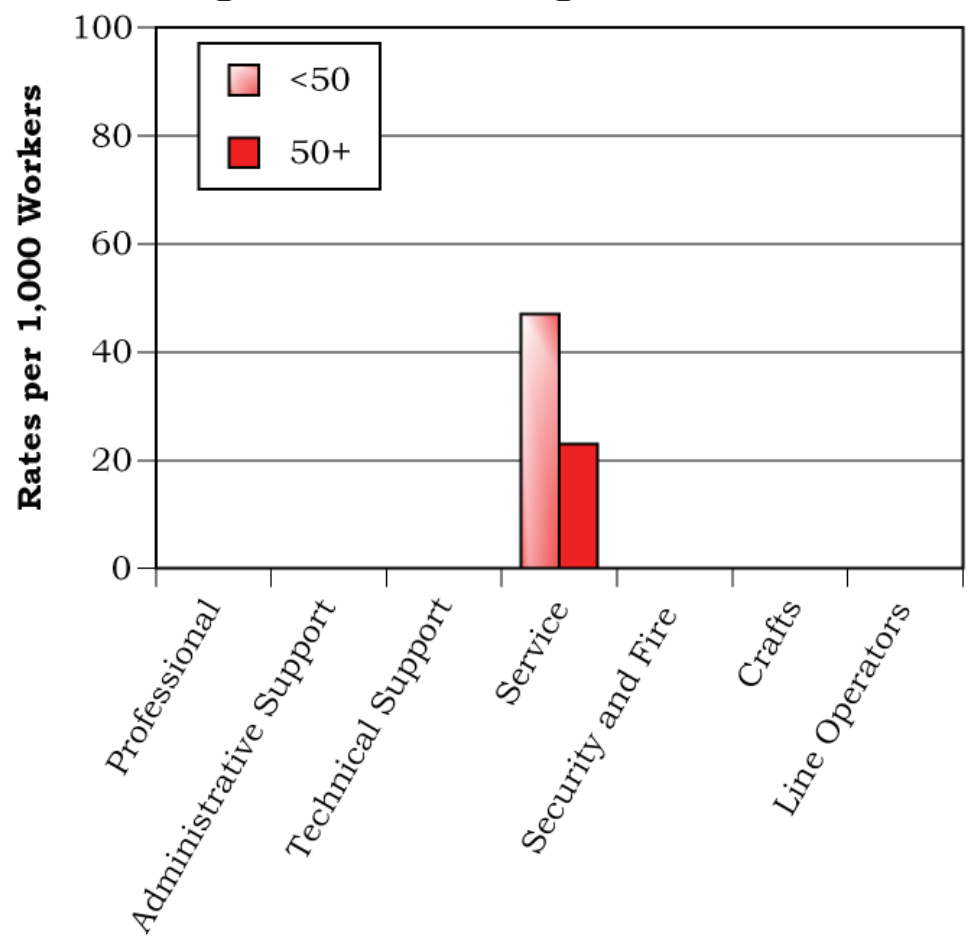

Job Category

Figure 23. OSHA-Recordable Rates by Age and Job Categories Among Men, All Diagnoses Combined

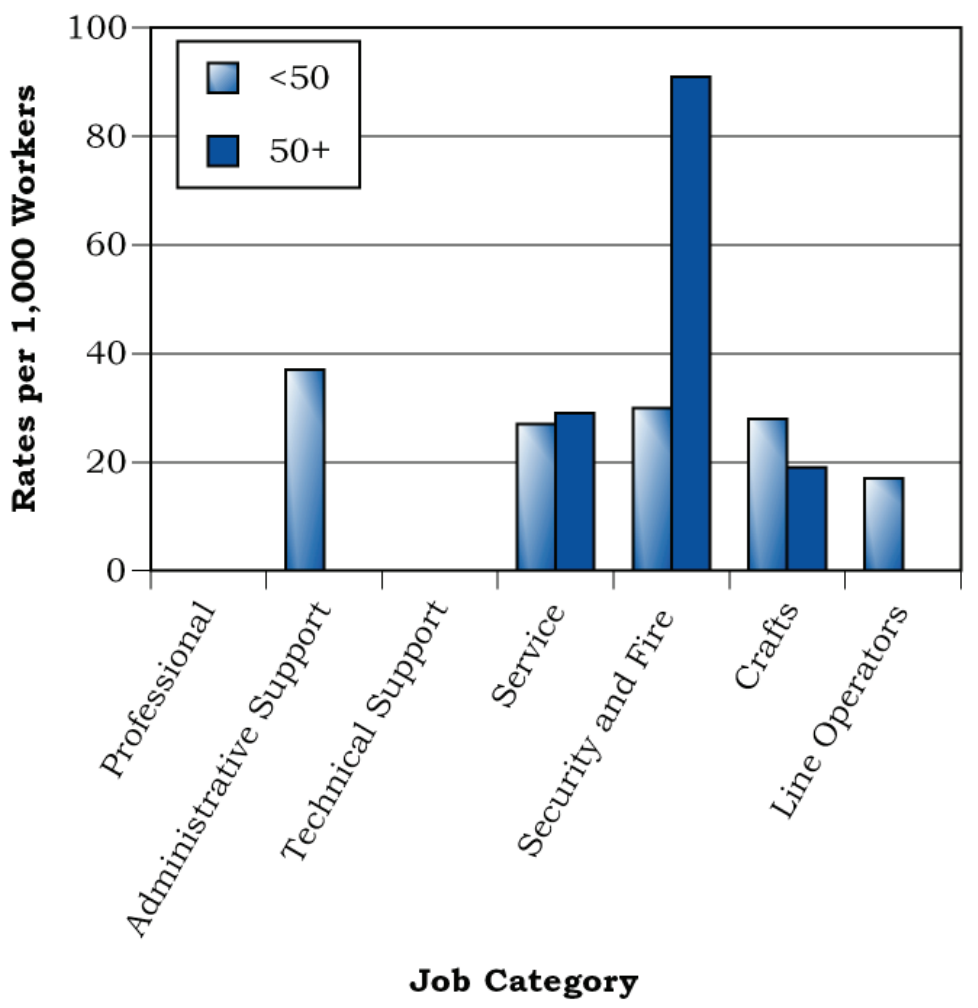


Time Trends for OSHA-Recordable Events

Figure 24. Age-Adjusted Rates for All OSHA-Recordable Diagnoses Combined Among Women and Men by Job Category from 2003 to 2007*

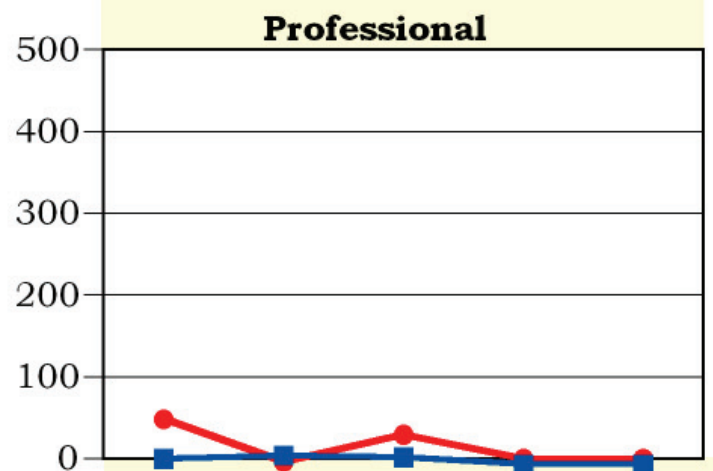

Administrative Support

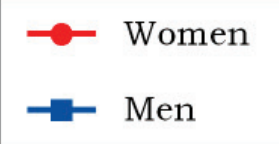

Technical Support

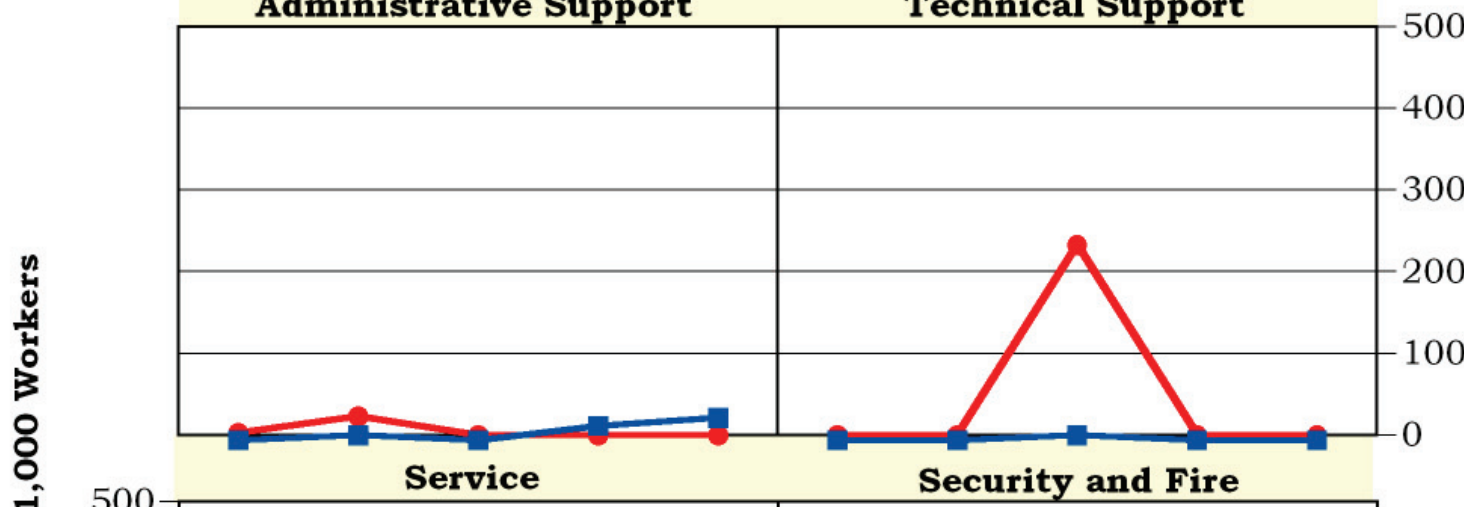

㟧

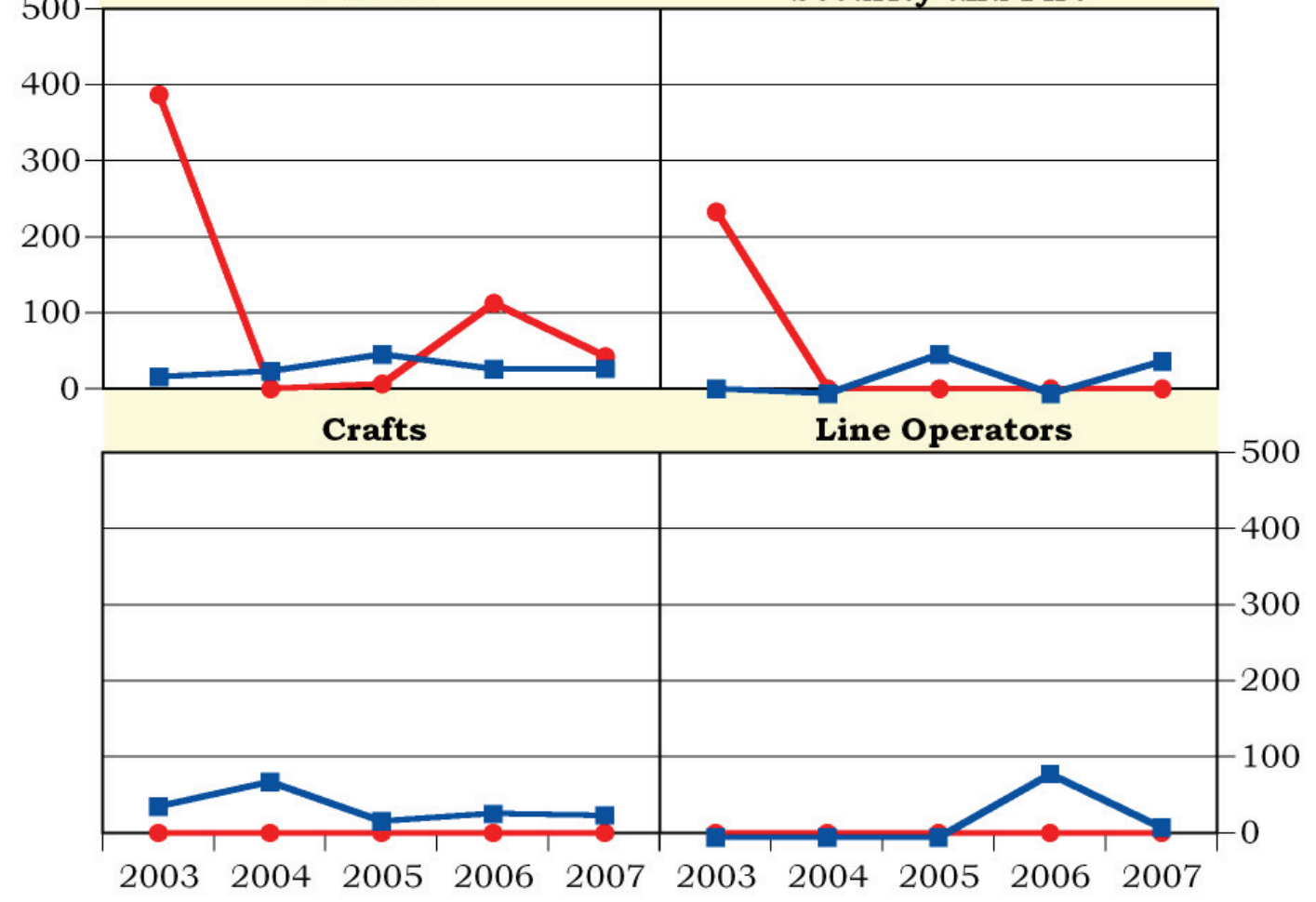

Year

* Standardized to age distribution of 2000 U.S. population. 


\section{Appendices}


East Tennessee Technology Park 2007

Absence Data

Appendix A. Work Force by Gender, Age, and Job Category

\begin{tabular}{|c|c|c|c|c|c|c|c|c|c|c|c|}
\hline \multirow{3}{*}{ Job Category } & \multicolumn{5}{|c|}{ Women } & \multicolumn{5}{|c|}{ Men } & \multirow[b]{3}{*}{ TOTAL } \\
\hline & \multicolumn{4}{|c|}{ Age Group } & \multirow[b]{2}{*}{ TOTAL } & \multicolumn{4}{|c|}{ Age Group } & \multirow[b]{2}{*}{ TOTAL } & \\
\hline & $16-29$ & 30 - 39 & $40-49$ & $50+$ & & $16-29$ & 30 - 39 & $40-49$ & $50+$ & & \\
\hline Professional & 3 & 19 & 53 & 60 & 135 & 15 & 33 & 149 & 342 & 539 & 674 \\
\hline Administrative Support & 4 & 21 & 47 & 74 & 146 & 8 & 8 & 11 & 35 & 62 & 208 \\
\hline Technical Support & 0 & 2 & 4 & 2 & 8 & 0 & 3 & 3 & 3 & 9 & 17 \\
\hline Service & 24 & 32 & 51 & 43 & 150 & 131 & 103 & 94 & 103 & 431 & 581 \\
\hline Security and Fire & 0 & 1 & 3 & 0 & 4 & 7 & 15 & 11 & 11 & 44 & 48 \\
\hline Crafts & 3 & 2 & 3 & 2 & 10 & 59 & 54 & 66 & 104 & 283 & 293 \\
\hline Line Operators & 0 & 0 & 3 & 6 & 9 & 7 & 20 & 32 & 53 & 112 & 121 \\
\hline TOTAL & 34 & 77 & 164 & 187 & 462 & 227 & 236 & 366 & 651 & 1,480 & 1,942 \\
\hline
\end{tabular}

Appendix B. Age Distribution of the Work Force by Gender

\begin{tabular}{|l|r|r|r|r|r|r|r|r|}
\hline \multirow{3}{*}{ Year } & \multicolumn{4}{|c|}{ Women } & \multicolumn{4}{c|}{ Men } \\
\cline { 2 - 9 } & \multicolumn{2}{|c|}{ Percent Distribution by Age Group } & \multicolumn{1}{c|}{ Percent Distribution by Age Group } \\
\cline { 2 - 9 } & $\mathbf{1 6}-\mathbf{2 9}$ & $\mathbf{3 0}-\mathbf{3 9}$ & $\mathbf{4 0}-\mathbf{4 9}$ & $\mathbf{5 0}+$ & $\mathbf{1 6}-\mathbf{2 9}$ & $\mathbf{3 0}-\mathbf{3 9}$ & $\mathbf{4 0}-\mathbf{4 9}$ & $\mathbf{5 0}+$ \\
\hline $\mathbf{1 9 9 9}$ & 5.50 & 27.02 & 43.53 & 23.95 & 2.10 & 15.08 & 39.76 & 43.06 \\
\hline $\mathbf{2 0 0 0}$ & 3.29 & 23.04 & 45.57 & 28.10 & 1.16 & 13.53 & 38.27 & 47.04 \\
\hline $\mathbf{2 0 0 1}$ & 3.19 & 22.36 & 43.13 & 31.31 & 1.39 & 11.44 & 33.69 & 53.48 \\
\hline $\mathbf{2 0 0 2}$ & 4.09 & 20.75 & 42.14 & 33.02 & 3.97 & 11.15 & 30.69 & 54.20 \\
\hline $\mathbf{2 0 0 3}$ & 4.69 & 19.06 & 42.19 & 34.06 & 3.24 & 10.96 & 33.02 & 52.78 \\
\hline $\mathbf{2 0 0 4}$ & 4.88 & 19.54 & 40.36 & 35.22 & 11.00 & 13.87 & 27.34 & 47.79 \\
\hline $\mathbf{2 0 0 5}$ & 6.62 & 17.89 & 41.18 & 34.31 & 13.95 & 14.30 & 27.13 & 44.62 \\
\hline $\mathbf{2 0 0 6}$ & 6.87 & 17.52 & 35.25 & 40.35 & 14.70 & 15.82 & 25.67 & 43.81 \\
\hline $\mathbf{2 0 0 7}$ & 7.36 & 16.67 & 35.50 & 40.48 & 15.34 & 15.95 & 24.73 & 43.99 \\
\hline
\end{tabular}


East Tennessee Technology Park 2007

Absence Data

Appendix C. Total Number of Workers Who Reported at Least One Absence by Gender, Age, and Job Category*

\begin{tabular}{|c|c|c|c|c|c|c|c|c|c|c|c|}
\hline \multirow{3}{*}{ Job Category } & \multicolumn{5}{|c|}{ Women } & \multicolumn{5}{|c|}{ Men } & \multirow[b]{3}{*}{ TOTAL } \\
\hline & \multicolumn{4}{|c|}{ Age Group } & \multirow[b]{2}{*}{ TOTAL } & \multicolumn{4}{|c|}{ Age Group } & \multirow[b]{2}{*}{ TOTAL } & \\
\hline & $16-29$ & 30 - 39 & $40-49$ & $50+$ & & 16 - 29 & 30 - 39 & $40-49$ & $50+$ & & \\
\hline Professional & 0 & 0 & 2 & 6 & 8 & 1 & 2 & 9 & 7 & 19 & 27 \\
\hline Administrative Support & 1 & 2 & 4 & 7 & 14 & 0 & 0 & 0 & 2 & 2 & 16 \\
\hline Technical Support & 0 & 1 & 1 & 0 & 2 & 0 & 0 & 0 & 1 & 1 & 3 \\
\hline Service & 0 & 0 & 0 & 1 & 1 & 1 & 1 & 2 & 7 & 11 & 12 \\
\hline Security and Fire & 0 & 0 & 1 & 0 & 1 & 1 & 1 & 1 & 0 & 3 & 4 \\
\hline Crafts & 0 & 0 & 0 & 0 & 0 & 0 & 0 & 2 & 7 & 9 & 9 \\
\hline Line Operators & 0 & 0 & 1 & 0 & 1 & 0 & 0 & 2 & 1 & 3 & 4 \\
\hline TOTAL & 1 & 3 & 9 & 14 & 27 & 3 & 4 & 16 & 25 & 48 & 75 \\
\hline
\end{tabular}

*Only those job categories and gender/age combinations with at least one absence appear in this table.

Appendix D. Total Number of Absences by Gender, Age, and Job Category*

\begin{tabular}{|c|c|c|c|c|c|c|c|c|c|c|c|}
\hline \multirow{3}{*}{ Job Category } & \multicolumn{5}{|c|}{ Women } & \multicolumn{5}{|c|}{ Men } & \multirow[b]{3}{*}{ TOTAL } \\
\hline & \multicolumn{4}{|c|}{ Age Group } & \multirow[b]{2}{*}{ TOTAL } & \multicolumn{4}{|c|}{ Age Group } & \multirow[b]{2}{*}{ TOTAL } & \\
\hline & $16-29$ & 30 - 39 & $40-49$ & $50+$ & & $16-29$ & 30 - 39 & $40-49$ & $50+$ & & \\
\hline Professional & 0 & 0 & 2 & 6 & 8 & 1 & 2 & 9 & 7 & 19 & 27 \\
\hline Administrative Support & 1 & 2 & 4 & 7 & 14 & 0 & 0 & 0 & 2 & 2 & 16 \\
\hline Technical Support & 0 & 1 & 1 & 0 & 2 & 0 & 0 & 0 & 1 & 1 & 3 \\
\hline Service & 0 & 0 & 0 & 2 & 2 & 1 & 1 & 2 & 7 & 11 & 13 \\
\hline Security and Fire & 0 & 0 & 1 & 0 & 1 & 1 & 1 & 1 & 0 & 3 & 4 \\
\hline Crafts & 0 & 0 & 0 & 0 & 0 & 0 & 0 & 2 & 8 & 10 & 10 \\
\hline Line Operators & 0 & 0 & 2 & 0 & 2 & 0 & 0 & 2 & 1 & 3 & 5 \\
\hline TOTAL & 1 & 3 & 10 & 15 & 29 & 3 & 4 & 16 & 26 & 49 & 78 \\
\hline
\end{tabular}

*Only those job categories and gender/age combinations with at least one absence appear in this table. 
East Tennessee Technology Park 2007

Absence Data

Appendix E. Distribution of the Number of Calendar Days Missed per Absence by Gender and Age*

\begin{tabular}{|c|c|c|c|c|c|c|c|c|c|c|c|}
\hline \multirow{3}{*}{$\begin{array}{c}\text { Number of } \\
\text { Calendar Days }\end{array}$} & \multicolumn{5}{|c|}{ Women } & \multicolumn{5}{|c|}{ Men } & \multirow[b]{3}{*}{ TOTAL } \\
\hline & \multicolumn{4}{|c|}{ Age Group } & \multirow[b]{2}{*}{ TOTAL } & \multicolumn{4}{|c|}{ Age Group } & \multirow[b]{2}{*}{ TOTAL } & \\
\hline & $16-29$ & $30-39$ & $40-49$ & $50+$ & & $16-29$ & $30-39$ & $40-49$ & $50+$ & & \\
\hline$<15$ & 0 & 0 & 3 & 4 & 7 & 1 & 0 & 5 & 9 & 15 & 22 \\
\hline $15-28$ & 1 & 1 & 2 & 4 & 8 & 1 & 2 & 4 & 5 & 12 & 20 \\
\hline $29-42$ & 0 & 0 & 2 & 1 & 3 & 0 & 0 & 1 & 4 & 5 & 8 \\
\hline $43-56$ & 0 & 1 & 1 & 1 & 3 & 0 & 1 & 0 & 1 & 2 & 5 \\
\hline $57-91$ & 0 & 1 & 0 & 4 & 5 & 1 & 0 & 2 & 3 & 6 & 11 \\
\hline $92-182$ & 0 & 0 & 2 & 1 & 3 & 0 & 1 & 4 & 4 & 9 & 12 \\
\hline TOTAL & 1 & 3 & 10 & 15 & 29 & 3 & 4 & 16 & 26 & 49 & 78 \\
\hline
\end{tabular}

*Only those gender/age combinations with at least one absence appear in this table. 
East Tennessee Technology Park 2007

Absence Data

Appendix F. Distribution of the Number of Calendar Days Missed per Absence by Gender and Job Category*

Women

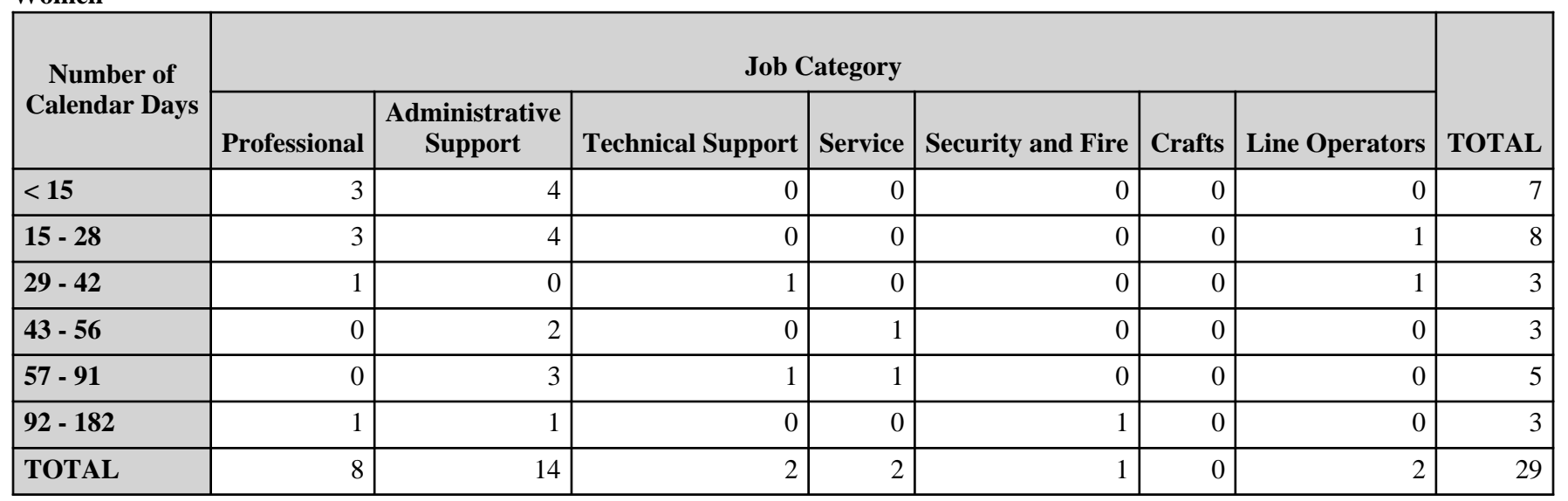

Men

\begin{tabular}{|c|c|c|c|c|c|c|c|c|}
\hline \multirow{2}{*}{$\begin{array}{c}\text { Number of } \\
\text { Calendar Days }\end{array}$} & \multicolumn{7}{|c|}{ Job Category } & \multirow[b]{2}{*}{ TOTAL } \\
\hline & Professional & $\begin{array}{c}\text { Administrative } \\
\text { Support }\end{array}$ & Technical Support & Service & Security and Fire & Crafts & Line Operators & \\
\hline$<15$ & 5 & 0 & 0 & 6 & 0 & 3 & 1 & 15 \\
\hline $15-28$ & 7 & 0 & 0 & 4 & 0 & 0 & 1 & 12 \\
\hline $43-56$ & 1 & 0 & 1 & 0 & 0 & 0 & 0 & 2 \\
\hline $57-91$ & 2 & 0 & 0 & 1 & 1 & 1 & 1 & 6 \\
\hline $92-182$ & 1 & 2 & 0 & 0 & 2 & 4 & 0 & 9 \\
\hline TOTAL & 19 & 2 & 1 & 11 & 3 & 10 & 3 & 49 \\
\hline
\end{tabular}

*Only those gender/job category combinations with at least one absence appear in this table. 
East Tennessee Technology Park 2007

Absence Data

Appendix G. Number of Diagnoses in Each Diagnostic Category by Gender and Age*

\begin{tabular}{|c|c|c|c|c|c|c|}
\hline & & \multicolumn{5}{|c|}{ Women } \\
\hline & & \multicolumn{4}{|c|}{ Age Group } & \multirow[b]{2}{*}{ TOTAL } \\
\hline & & $16-29$ & $30-39$ & $40-49$ & $50+$ & \\
\hline Diagnostic Category & ICD-9-CM Code & \multirow[b]{2}{*}{0} & \multirow[b]{2}{*}{0} & \multirow[b]{2}{*}{0} & \multirow[b]{2}{*}{1} & \multirow[b]{2}{*}{1} \\
\hline MALIGNANT NEOPLASMS & $140-208,230-234$ & & & & & \\
\hline -Bone, Connective Tissue, Skin & $170-173,176$ & 0 & 0 & 0 & 0 & 0 \\
\hline -Genitourinary & 179-189 & 0 & 0 & 0 & 0 & 0 \\
\hline -Carcinoma in situ & $230-234$ & 0 & 0 & 0 & 1 & 1 \\
\hline ENDOCRINE/METABOLIC/IMMUNITY & $240-279$ & 0 & 0 & 0 & 1 & 1 \\
\hline -Thyroid Gland Disorders & $240-246$ & 0 & 0 & 0 & 1 & 1 \\
\hline -Other Endocrine Gland Dis & $250-259$ & 0 & 0 & 0 & 0 & 0 \\
\hline MENTAL DISORDERS & $290-319$ & 0 & 0 & 0 & 0 & 0 \\
\hline -Non-Psychotic Disorders & $300-302,306-316$ & 0 & 0 & 0 & 0 & 0 \\
\hline NERVOUS SYSTEM (NS) \& SENSE ORGANS & 320-389 & 0 & 1 & 2 & 0 & 3 \\
\hline -Other Disorders of Central NS & $340-349$ & 0 & 1 & 0 & 0 & 1 \\
\hline -Disorders of Peripheral NS & 350-359 & 0 & 0 & 2 & 0 & 2 \\
\hline -Disorders of Eye & $360-379$ & 0 & 0 & 0 & 0 & 0 \\
\hline -Dis of Ear \& Mastoid & $380-389$ & 0 & 0 & 0 & 0 & 0 \\
\hline CIRCULATORY SYSTEM & $390-459$ & 0 & 0 & 0 & 1 & 1 \\
\hline -Ischemic Heart Dis & $410-414$ & 0 & 0 & 0 & 0 & 0 \\
\hline -Other Heart Dis & $420-429$ & 0 & 0 & 0 & 0 & 0 \\
\hline -Cerebrovascular Dis & $430-438$ & 0 & 0 & 0 & 1 & 1 \\
\hline -Dis of Veins, Lymphatics, Other & $451-459$ & 0 & 0 & 0 & 0 & 0 \\
\hline RESPIRATORY SYSTEM & $460-519$ & 0 & 0 & 0 & 3 & 3 \\
\hline -Acute Respiratory Infections & $460-466$ & 0 & 0 & 0 & 1 & 1 \\
\hline -Other Dis Upper Respiratory Tract & $470-478$ & 0 & 0 & 0 & 2 & 2 \\
\hline -Pneumonia \& Influenza & $480-487$ & 0 & 0 & 0 & 0 & 0 \\
\hline -Chronic Obstructive Dis & $490-496$ & 0 & 0 & 0 & 0 & 0 \\
\hline DIGESTIVE SYSTEM & $520-579$ & 0 & 0 & 2 & 2 & 4 \\
\hline -Oral Cavity, Saliva Glands, Jaw & $520-529$ & 0 & 0 & 0 & 0 & 0 \\
\hline -Esophagus, Stomach, Duodenum & $530-537$ & 0 & 0 & 0 & 0 & 0 \\
\hline -Appendicitis & $540-543$ & 0 & 0 & 0 & 0 & 0 \\
\hline -Hernia & $550-553$ & 0 & 0 & 0 & 1 & 1 \\
\hline -Other Intestinal Dis & $560-569$ & 0 & 0 & 0 & 0 & 0 \\
\hline -Other Digestive Dis & $570-579$ & 0 & 0 & 2 & 1 & 3 \\
\hline GENITOURINARY SYSTEM & $580-629$ & 0 & 3 & 3 & 2 & 8 \\
\hline -Other Urinary Dis & $590-599$ & 0 & 0 & 1 & 0 & 1 \\
\hline -Male Genital Organ Dis & $600-608$ & 0 & 0 & 0 & 0 & 0 \\
\hline -Other Female Disorders & $617-629$ & 0 & 3 & 2 & 2 & 7 \\
\hline SKIN \& SUBCUTANEOUS TISSUE & 680-709 & 0 & 0 & 0 & 1 & 1 \\
\hline -Other & 700-709 & 0 & 0 & 0 & 1 & 1 \\
\hline
\end{tabular}

*Only those diagnostic categories and gender/age combinations with at least one occurrence appear in this table. 
East Tennessee Technology Park 2007

Absence Data

Appendix G. Number of Diagnoses in Each Diagnostic Category by Gender and Age*

\begin{tabular}{|c|c|c|c|c|c|c|c|}
\hline & & \multicolumn{5}{|c|}{ Men } & \multirow[b]{3}{*}{ TOTAL } \\
\hline & & \multicolumn{4}{|c|}{ Age Group } & \multirow[b]{2}{*}{ TOTAL } & \\
\hline & & $16-29$ & $30-39$ & $40-49$ & $50+$ & & \\
\hline MALIGNANT NEOPLASMS & $140-208,230-234$ & 0 & 0 & 1 & 3 & 4 & 5 \\
\hline -Bone, Connective Tissue, Skin & $170-173,176$ & 0 & 0 & 0 & 1 & 1 & 1 \\
\hline -Carcinoma in situ & $230-234$ & 0 & 0 & 0 & 0 & 0 & 1 \\
\hline ENDOCRINE/METABOLIC/IMMUNITY & $240-279$ & 0 & 0 & 0 & 1 & 1 & 2 \\
\hline -Thyroid Gland Disorders & $240-246$ & 0 & 0 & 0 & 0 & 0 & 1 \\
\hline -Other Endocrine Gland Dis & $250-259$ & 0 & 0 & 0 & 1 & 1 & 1 \\
\hline -Other Disorders of Central NS & $340-349$ & 0 & 0 & 0 & 0 & 0 & 1 \\
\hline -Disorders of Peripheral NS & $350-359$ & 0 & 0 & 1 & 0 & 1 & 3 \\
\hline -Disorders of Eye & $360-379$ & 0 & 0 & 0 & 3 & 3 & 3 \\
\hline -Dis of Ear \& Mastoid & 380-389 & 0 & 0 & 0 & 1 & 1 & 1 \\
\hline CIRCULATORY SYSTEM & $390-459$ & 0 & 0 & 1 & 4 & 5 & 6 \\
\hline -Ischemic Heart Dis & $410-414$ & 0 & 0 & 0 & 1 & 1 & 1 \\
\hline -Other Heart Dis & $420-429$ & 0 & 0 & 0 & 1 & 1 & 1 \\
\hline -Cerebrovascular Dis & $430-438$ & 0 & 0 & 0 & 0 & 0 & 1 \\
\hline -Chronic Obstructive Dis & $490-496$ & 0 & 0 & 0 & 1 & 1 & 1 \\
\hline DIGESTIVE SYSTEM & $520-579$ & 0 & 2 & 3 & 1 & 6 & 10 \\
\hline -Oral Cavity, Saliva Glands, Jaw & $520-529$ & 0 & 0 & 0 & 1 & 1 & 1 \\
\hline -Esophagus, Stomach, Duodenum & $\mathbf{5 3 0 - 5 3 7}$ & 0 & 1 & 0 & 0 & 1 & 1 \\
\hline -Appendicitis & $540-543$ & 0 & 0 & 1 & 0 & 1 & 1 \\
\hline -Hernia & $550-553$ & 0 & 1 & 0 & 0 & 1 & 2 \\
\hline -Other Intestinal Dis & $560-569$ & 0 & 0 & 2 & 0 & 2 & 2 \\
\hline -Other Digestive Dis & $570-579$ & 0 & 0 & 0 & 0 & 0 & 3 \\
\hline GENITOURINARY SYSTEM & $580-629$ & 0 & 0 & 0 & 1 & 1 & 9 \\
\hline -Other Urinary Dis & $590-599$ & 0 & 0 & 0 & 0 & 0 & 1 \\
\hline -Male Genital Organ Dis & $600-608$ & 0 & 0 & 0 & 1 & 1 & 1 \\
\hline -Other Female Disorders & $617-629$ & 0 & 0 & 0 & 0 & 0 & 7 \\
\hline SKIN \& SUBCUTANEOUS TISSUE & 680-709 & 0 & 0 & 0 & 0 & 0 & 1 \\
\hline -Other & 700-709 & 0 & 0 & 0 & 0 & 0 & 1 \\
\hline
\end{tabular}

(Continued)

*Only those diagnostic categories and gender/age combinations with at least one occurrence appear in this table. 
East Tennessee Technology Park 2007

Absence Data

Appendix G. Number of Diagnoses in Each Diagnostic Category by Gender and Age*

\begin{tabular}{|c|c|c|c|c|c|c|}
\hline & & \multicolumn{5}{|c|}{ Women } \\
\hline & & \multicolumn{4}{|c|}{ Age Group } & \multirow[b]{2}{*}{ TOTAL } \\
\hline & & $16-29$ & 30 - 39 & $40-49$ & $\mathbf{5 0 +}$ & \\
\hline Diagnostic Category & ICD-9-CM Code & & & & & \\
\hline $\begin{array}{l}\text { MUSCULOSKELETAL \& CONNECTIVE } \\
\text { TISSUE }\end{array}$ & $710-739$ & 1 & 1 & 3 & 3 & 8 \\
\hline -Arthropathies & 710-719 & 0 & 0 & 1 & 0 & 1 \\
\hline -Dorsopathies & $720-724$ & 0 & 1 & 1 & 0 & 2 \\
\hline -Rheumatism, Excluding Back & $725-729$ & 0 & 0 & 1 & 3 & 4 \\
\hline -Other Dis \& Acquired Deformities & $730-739$ & 1 & 0 & 0 & 0 & 1 \\
\hline CONGENITAL ANOMALIES & 740-759 & 0 & 0 & 1 & 0 & 1 \\
\hline $\begin{array}{l}\text { SYMPTOMS, SIGNS, \& ILL-DEFINED } \\
\text { CONDITIONS }\end{array}$ & $780-799$ & 0 & 0 & 0 & 0 & 0 \\
\hline -Symptoms & 780-789 & 0 & 0 & 0 & 0 & 0 \\
\hline INJURY \& POISONING & $800-999$ & 0 & 0 & 0 & 4 & 4 \\
\hline -Fracture - Skull & $800-804$ & 0 & 0 & 0 & 0 & 0 \\
\hline -Fracture - Neck, Trunk & 805-809 & 0 & 0 & 0 & 0 & 0 \\
\hline -Fracture - Upper Limb & 810-819 & 0 & 0 & 0 & 0 & 0 \\
\hline -Fracture - Lower Limb & $820-829$ & 0 & 0 & 0 & 2 & 2 \\
\hline -Dislocation & $830-839$ & 0 & 0 & 0 & 0 & 0 \\
\hline -Sprains \& Strains - Back & 846-847 & 0 & 0 & 0 & 1 & 1 \\
\hline -Sprains \& Strains - Other & $840-845,848$ & 0 & 0 & 0 & 1 & 1 \\
\hline -Internal Injury - Thorax, Abdomen, Pelvis & $860-869$ & 0 & 0 & 0 & 0 & 0 \\
\hline -Open Wound - Head, Neck, Trunk & 870-879 & 0 & 0 & 0 & 0 & 0 \\
\hline -Open Wound - Lower Limb & 890-897 & 0 & 0 & 0 & 0 & 0 \\
\hline -Contusion & $920-924$ & 0 & 0 & 0 & 0 & 0 \\
\hline -Complications \& Unspecified Injuries & 958-959 & 0 & 0 & 0 & 0 & 0 \\
\hline -Complications of Surgical/Medical Care & 996-999 & 0 & 0 & 0 & 0 & 0 \\
\hline
\end{tabular}

*Only those diagnostic categories and gender/age combinations with at least one occurrence appear in this table. 
East Tennessee Technology Park 2007

Absence Data

Appendix G. Number of Diagnoses in Each Diagnostic Category by Gender and Age*

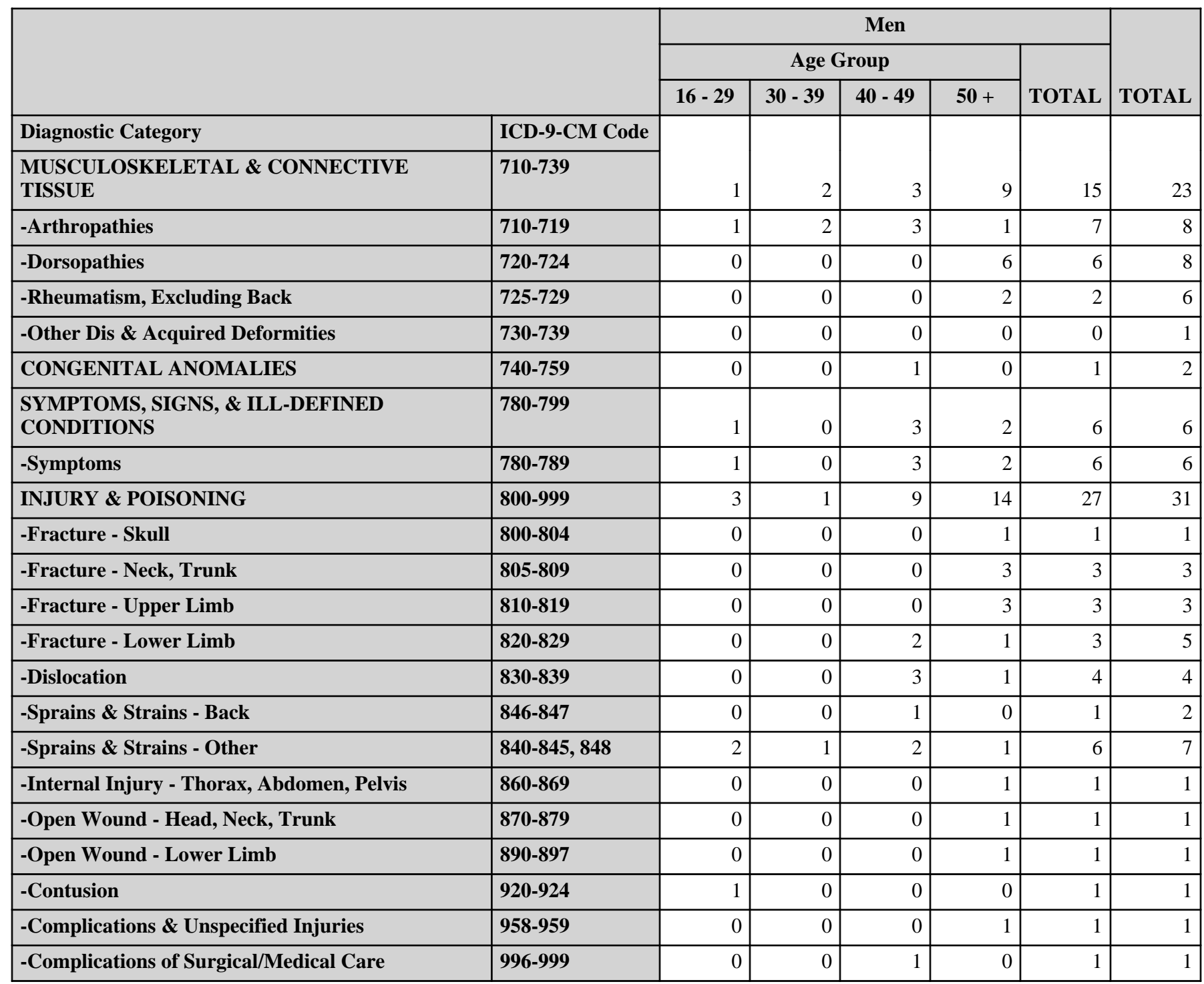

\begin{tabular}{|c|c|c|c|c|c|c|c|c|c|c|c|}
\hline & \multicolumn{5}{|c|}{ Women } & \multicolumn{5}{|c|}{ Men } & \multirow[b]{3}{*}{ TOTAL } \\
\hline & \multicolumn{4}{|c|}{ Age Group } & \multirow[b]{2}{*}{ TOTAL } & \multicolumn{4}{|c|}{ Age Group } & \multirow[b]{2}{*}{ TOTAL } & \\
\hline & $16-29$ & $30-39$ & $40-49$ & $50+$ & & $16-29$ & $30-39$ & $40-49$ & $50+$ & & \\
\hline Diagnostic Category & \multirow[b]{2}{*}{1} & \multirow[b]{2}{*}{5} & \multirow[b]{2}{*}{11} & \multirow[b]{2}{*}{18} & \multirow[b]{2}{*}{35} & \multirow[b]{2}{*}{5} & \multirow[b]{2}{*}{5} & \multirow[b]{2}{*}{23} & \multirow[b]{2}{*}{45} & \multirow[b]{2}{*}{78} & \multirow[b]{2}{*}{113} \\
\hline Total & & & & & & & & & & & \\
\hline
\end{tabular}

*Only those diagnostic categories and gender/age combinations with at least one occurrence appear in this table. 
East Tennessee Technology Park 2007

Absence Data

Appendix H. Total Number of Calendar Days Absent in Each Diagnostic Category by Gender and Age*

\begin{tabular}{|c|c|c|c|c|c|c|}
\hline & \multicolumn{5}{|c|}{ Women } \\
\hline & & \multicolumn{4}{|c|}{ Age Group } & \multirow[b]{2}{*}{ TOTAL } \\
\hline & & $16-29$ & $30-39$ & $40-49$ & $50+$ & \\
\hline Diagnostic Category & ICD-9-CM Code & \multirow[b]{2}{*}{0} & \multirow[b]{2}{*}{0} & \multirow[b]{2}{*}{0} & \multirow[b]{2}{*}{68} & \multirow[b]{2}{*}{68} \\
\hline MALIGNANT NEOPLASMS & 140-208, 230-234 & & & & & \\
\hline ENDOCRINE/METABOLIC/IMMUNITY & 240-279 & 0 & 0 & 0 & 18 & 18 \\
\hline MENTAL DISORDERS & $290-319$ & 0 & 0 & 0 & 0 & 0 \\
\hline NERVOUS SYSTEM (NS) \& SENSE ORGANS & $320-389$ & 0 & 70 & 52 & 0 & 122 \\
\hline CIRCULATORY SYSTEM & $390-459$ & 0 & 0 & 0 & 34 & 34 \\
\hline RESPIRATORY SYSTEM & $460-519$ & 0 & 0 & 0 & 64 & 64 \\
\hline DIGESTIVE SYSTEM & $520-579$ & 0 & 0 & 30 & 62 & 92 \\
\hline GENITOURINARY SYSTEM & $\begin{array}{l}580-629 \\
\end{array}$ & 0 & 17 & 71 & 33 & 121 \\
\hline SKIN \& SUBCUTANEOUS TISSUE & 680-709 & 0 & 0 & 0 & 20 & 20 \\
\hline $\begin{array}{l}\text { MUSCULOSKELETAL \& CONNECTIVE } \\
\text { TISSUE }\end{array}$ & 710-739 & 18 & 49 & 144 & 67 & 278 \\
\hline CONGENITAL ANOMALIES & 740-759 & 0 & 0 & 130 & 0 & 130 \\
\hline $\begin{array}{l}\text { SYMPTOMS, SIGNS, \& ILL-DEFINED } \\
\text { CONDITIONS }\end{array}$ & 780-799 & 0 & 0 & 0 & 0 & 0 \\
\hline INJURY \& POISONING & $800-999$ & 0 & 0 & 0 & 192 & 192 \\
\hline
\end{tabular}

\begin{tabular}{|c|c|c|c|c|c|c|c|}
\hline & \multicolumn{5}{|c|}{ Men } & \multirow[b]{3}{*}{ TOTAL } \\
\hline & & \multicolumn{4}{|c|}{ Age Group } & \multirow[b]{2}{*}{ TOTAL } & \\
\hline & & $16-29$ & $30-39$ & $40-49$ & $50+$ & & \\
\hline Diagnostic Category & ICD-9-CM Code & \multirow[b]{2}{*}{0} & \multirow[b]{2}{*}{0} & \multirow[b]{2}{*}{18} & \multirow[b]{2}{*}{68} & \multirow[b]{2}{*}{86} & \multirow[b]{2}{*}{154} \\
\hline MALIGNANT NEOPLASMS & $140-208,230-234$ & & & & & & \\
\hline ENDOCRINE/METABOLIC/IMMUNITY & 240-279 & 0 & 0 & 0 & 21 & 21 & 39 \\
\hline MENTAL DISORDERS & $290-319$ & 0 & 0 & 0 & 10 & 10 & 10 \\
\hline NERVOUS SYSTEM (NS) \& SENSE ORGANS & 320-389 & 0 & 0 & 18 & 28 & 46 & 168 \\
\hline CIRCULATORY SYSTEM & 390-459 & 0 & 0 & 21 & 180 & 201 & 235 \\
\hline RESPIRATORY SYSTEM & $460-519$ & 0 & 0 & 7 & 69 & 76 & 140 \\
\hline DIGESTIVE SYSTEM & 520-579 & 0 & 22 & 33 & 45 & 100 & 192 \\
\hline GENITOURINARY SYSTEM & 580-629 & 0 & 0 & 0 & 19 & 19 & 140 \\
\hline SKIN \& SUBCUTANEOUS TISSUE & 680-709 & 0 & 0 & 0 & 0 & 0 & 20 \\
\hline $\begin{array}{l}\text { MUSCULOSKELETAL \& CONNECTIVE } \\
\text { TISSUE }\end{array}$ & 710-739 & 2 & 165 & 134 & 396 & 697 & 975 \\
\hline CONGENITAL ANOMALIES & 740-759 & 0 & 0 & 82 & 0 & 82 & 212 \\
\hline $\begin{array}{l}\text { SYMPTOMS, SIGNS, \& ILL-DEFINED } \\
\text { CONDITIONS }\end{array}$ & 780-799 & 18 & 0 & 179 & 81 & 278 & 278 \\
\hline INJURY \& POISONING & $800-999$ & 90 & 25 & 509 & 329 & 953 & 1,145 \\
\hline
\end{tabular}

\footnotetext{
*Absences with >1 ICD-9-CM code in the same diagnostic category were counted only once. Only those diagnostic categories and gender/age combinations with at least one occurrence appear in this table.
} 
East Tennessee Technology Park 2007

Absence Data

Appendix I. Number of Diagnoses in Each Diagnostic Category by Gender and Job Category*

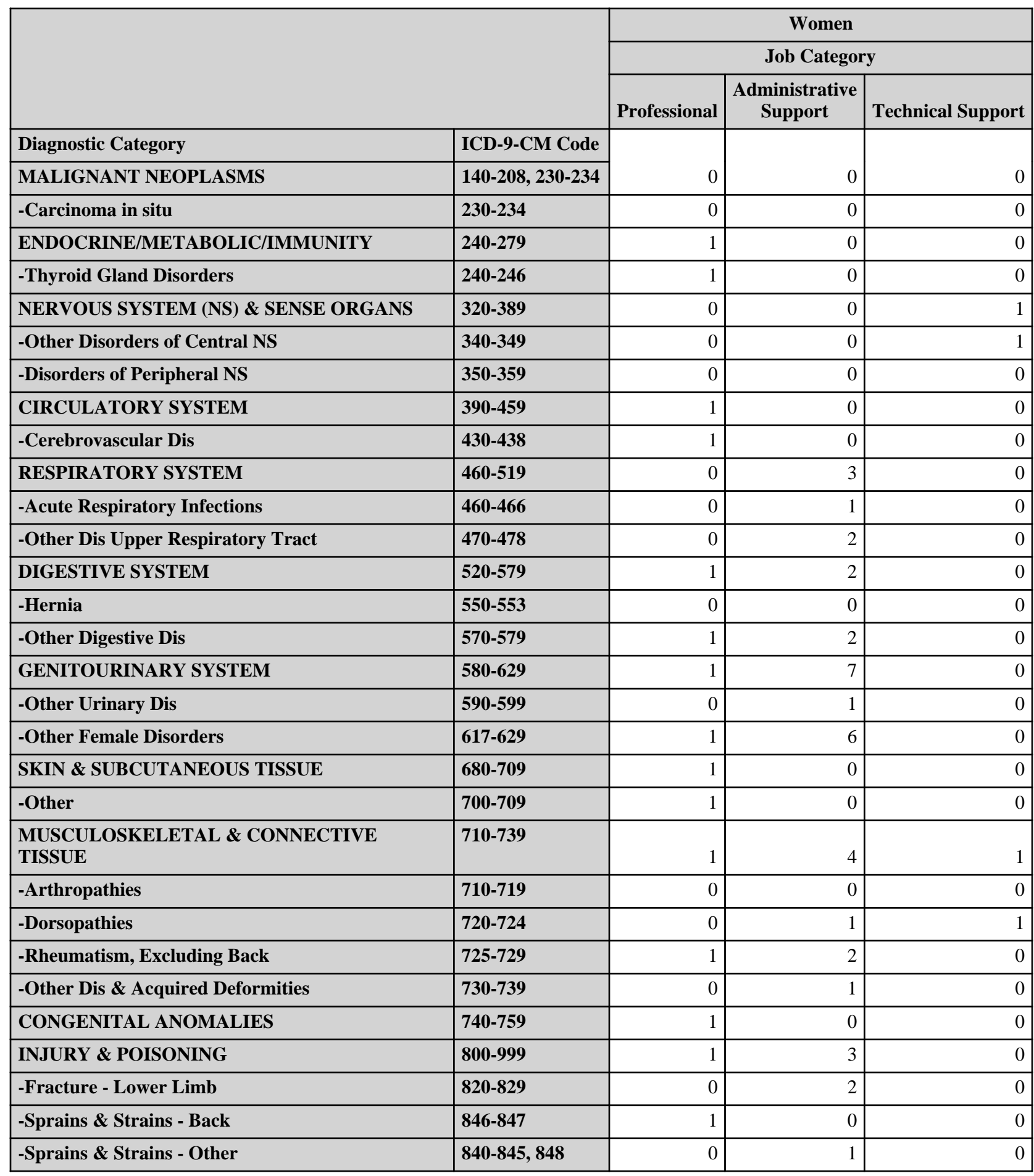

*Only those diagnostic categories and gender/job category combinations with at least one occurrence appear in this table. 
East Tennessee Technology Park 2007

Absence Data

Appendix I. Number of Diagnoses in Each Diagnostic Category by Gender and Job Category*

\begin{tabular}{|c|c|c|c|c|c|}
\hline & & \multicolumn{4}{|c|}{ Women } \\
\hline & & \multicolumn{3}{|c|}{ Job Category } & \multirow[b]{2}{*}{ TOTAL } \\
\hline & & Service & Security and Fire & Line Operators & \\
\hline Diagnostic Category & ICD-9-CM Code & & & & \\
\hline MALIGNANT NEOPLASMS & $140-208,230-234$ & 1 & 0 & 0 & 1 \\
\hline -Carcinoma in situ & $230-234$ & 1 & 0 & 0 & 1 \\
\hline ENDOCRINE/METABOLIC/IMMUNITY & 240-279 & 0 & 0 & 0 & 1 \\
\hline -Thyroid Gland Disorders & $240-246$ & 0 & 0 & 0 & 1 \\
\hline NERVOUS SYSTEM (NS) \& SENSE ORGANS & 320-389 & 0 & 0 & 2 & 3 \\
\hline -Other Disorders of Central NS & $340-349$ & 0 & 0 & 0 & 1 \\
\hline -Disorders of Peripheral NS & $350-359$ & 0 & 0 & 2 & 2 \\
\hline CIRCULATORY SYSTEM & $390-459$ & 0 & 0 & 0 & 1 \\
\hline -Cerebrovascular Dis & $430-438$ & 0 & 0 & 0 & 1 \\
\hline RESPIRATORY SYSTEM & $460-519$ & 0 & 0 & 0 & 3 \\
\hline -Acute Respiratory Infections & $460-466$ & 0 & 0 & 0 & 1 \\
\hline -Other Dis Upper Respiratory Tract & $470-478$ & 0 & 0 & 0 & 2 \\
\hline DIGESTIVE SYSTEM & $520-579$ & 1 & 0 & 0 & 4 \\
\hline -Hernia & $550-553$ & 1 & 0 & 0 & 1 \\
\hline -Other Digestive Dis & $570-579$ & 0 & 0 & 0 & 3 \\
\hline GENITOURINARY SYSTEM & $580-629$ & 0 & 0 & 0 & 8 \\
\hline -Other Urinary Dis & $590-599$ & 0 & 0 & 0 & 1 \\
\hline -Other Female Disorders & $617-629$ & 0 & 0 & 0 & 7 \\
\hline SKIN \& SUBCUTANEOUS TISSUE & 680-709 & 0 & 0 & 0 & 1 \\
\hline -Other & 700-709 & 0 & 0 & 0 & 1 \\
\hline $\begin{array}{l}\text { MUSCULOSKELETAL \& CONNECTIVE } \\
\text { TISSUE }\end{array}$ & $710-739$ & 0 & 2 & 0 & 8 \\
\hline -Arthropathies & $710-719$ & 0 & 1 & 0 & 1 \\
\hline -Dorsopathies & $720-724$ & 0 & 0 & 0 & 2 \\
\hline -Rheumatism, Excluding Back & 725-729 & 0 & 1 & 0 & 4 \\
\hline -Other Dis \& Acquired Deformities & 730-739 & 0 & 0 & 0 & 1 \\
\hline CONGENITAL ANOMALIES & 740-759 & 0 & 0 & 0 & 1 \\
\hline INJURY \& POISONING & 800-999 & 0 & 0 & 0 & 4 \\
\hline -Fracture - Lower Limb & 820-829 & 0 & 0 & 0 & 2 \\
\hline -Sprains \& Strains - Back & 846-847 & 0 & 0 & 0 & 1 \\
\hline -Sprains \& Strains - Other & $840-845,848$ & 0 & 0 & 0 & 1 \\
\hline
\end{tabular}

*Only those diagnostic categories and gender/job category combinations with at least one occurrence appear in this table. 
East Tennessee Technology Park 2007

Absence Data

Appendix I. Number of Diagnoses in Each Diagnostic Category by Gender and Job Category*

\begin{tabular}{|c|c|c|c|c|c|c|c|}
\hline & \multicolumn{7}{|c|}{ Women } \\
\hline & \multicolumn{6}{|c|}{ Job Category } & \multirow[b]{2}{*}{ TOTAL } \\
\hline & Professional & $\begin{array}{c}\text { Administrative } \\
\text { Support }\end{array}$ & Technical Support & Service & Security and Fire & Line Operators & \\
\hline Diagnostic Category & \multirow[b]{2}{*}{8} & \multirow[b]{2}{*}{19} & \multirow[b]{2}{*}{2} & \multirow[b]{2}{*}{2} & \multirow[b]{2}{*}{2} & \multirow[b]{2}{*}{2} & \multirow[b]{2}{*}{35} \\
\hline Total & & & & & & & \\
\hline
\end{tabular}

*Only those diagnostic categories and gender/job category combinations with at least one occurrence appear in this table. 
East Tennessee Technology Park 2007

Absence Data

Appendix I. Number of Diagnoses in Each Diagnostic Category by Gender and Job Category*

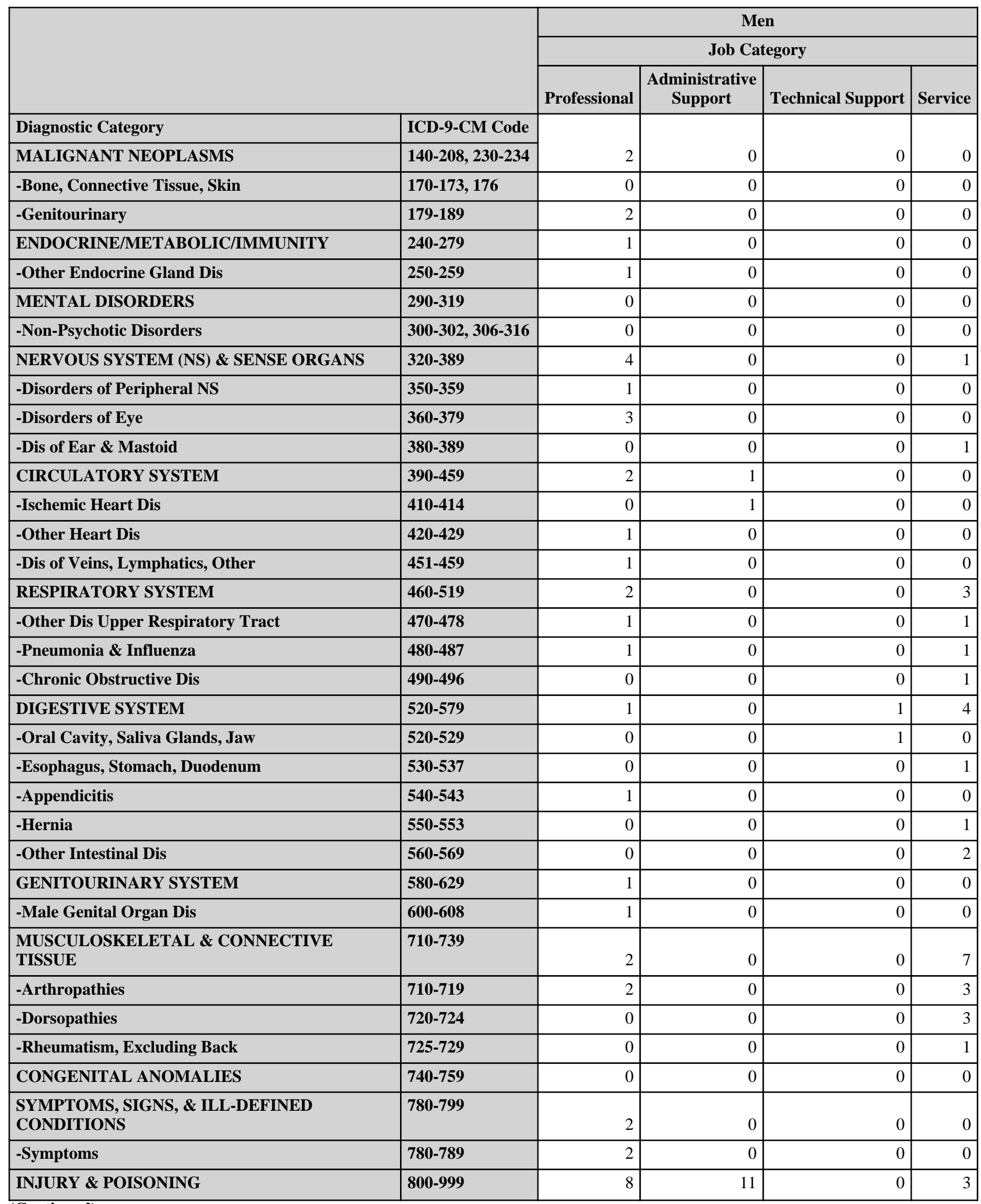

(Continued)

*Only those diagnostic categories and gender/job category combinations with at least one occurrence appear in this table. 
East Tennessee Technology Park 2007

Absence Data

Appendix I. Number of Diagnoses in Each Diagnostic Category by Gender and Job Category*

\begin{tabular}{|c|c|c|c|c|c|}
\hline & & \multicolumn{4}{|c|}{ Men } \\
\hline & & \multicolumn{3}{|c|}{ Job Category } & \multirow[b]{2}{*}{ TOTAL } \\
\hline & & Security and Fire & Crafts & Line Operators & \\
\hline Diagnostic Category & ICD-9-CM Code & \multirow[b]{2}{*}{0} & \multirow[b]{2}{*}{1} & \multirow[b]{2}{*}{1} & \multirow[b]{2}{*}{4} \\
\hline MALIGNANT NEOPLASMS & $140-208,230-234$ & & & & \\
\hline -Bone, Connective Tissue, Skin & $170-173,176$ & 0 & 1 & 0 & 1 \\
\hline -Genitourinary & 179-189 & 0 & 0 & 1 & 3 \\
\hline ENDOCRINE/METABOLIC/IMMUNITY & $240-279$ & 0 & 0 & 0 & 1 \\
\hline -Other Endocrine Gland Dis & $250-259$ & 0 & 0 & 0 & 1 \\
\hline MENTAL DISORDERS & 290-319 & 0 & 2 & 0 & 2 \\
\hline -Non-Psychotic Disorders & $300-302,306-316$ & 0 & 2 & 0 & 2 \\
\hline NERVOUS SYSTEM (NS) \& SENSE ORGANS & $320-389$ & 0 & 0 & 0 & 5 \\
\hline -Disorders of Peripheral NS & $350-359$ & 0 & 0 & 0 & 1 \\
\hline -Disorders of Eye & $360-379$ & 0 & 0 & 0 & 3 \\
\hline -Dis of Ear \& Mastoid & $380-389$ & 0 & 0 & 0 & 1 \\
\hline CIRCULATORY SYSTEM & $390-459$ & 0 & 2 & 0 & 5 \\
\hline -Ischemic Heart Dis & $410-414$ & 0 & 0 & 0 & 1 \\
\hline -Other Heart Dis & $420-429$ & 0 & 0 & 0 & 1 \\
\hline -Dis of Veins, Lymphatics, Other & $451-459$ & 0 & 2 & 0 & 3 \\
\hline RESPIRATORY SYSTEM & $460-519$ & 0 & 0 & 0 & 5 \\
\hline -Other Dis Upper Respiratory Tract & $470-478$ & 0 & 0 & 0 & 2 \\
\hline -Pneumonia \& Influenza & $480-487$ & 0 & 0 & 0 & 2 \\
\hline -Chronic Obstructive Dis & $490-496$ & 0 & 0 & 0 & 1 \\
\hline DIGESTIVE SYSTEM & $520-579$ & 0 & 0 & 0 & 6 \\
\hline -Oral Cavity, Saliva Glands, Jaw & $520-529$ & 0 & 0 & 0 & 1 \\
\hline -Esophagus, Stomach, Duodenum & $\mathbf{5 3 0 - 5 3 7}$ & 0 & 0 & 0 & 1 \\
\hline -Appendicitis & $540-543$ & 0 & 0 & 0 & 1 \\
\hline -Hernia & $550-553$ & 0 & 0 & 0 & 1 \\
\hline -Other Intestinal Dis & $560-569$ & 0 & 0 & 0 & 2 \\
\hline GENITOURINARY SYSTEM & $580-629$ & 0 & 0 & 0 & 1 \\
\hline -Male Genital Organ Dis & $600-608$ & 0 & 0 & 0 & 1 \\
\hline $\begin{array}{l}\text { MUSCULOSKELETAL \& CONNECTIVE } \\
\text { TISSUE }\end{array}$ & $710-739$ & 1 & 4 & 1 & 15 \\
\hline -Arthropathies & $710-719$ & 1 & 1 & 0 & 7 \\
\hline -Dorsopathies & $720-724$ & 0 & 2 & 1 & 6 \\
\hline -Rheumatism, Excluding Back & $725-729$ & 0 & 1 & 0 & 2 \\
\hline CONGENITAL ANOMALIES & $740-759$ & 0 & 0 & 1 & 1 \\
\hline $\begin{array}{l}\text { SYMPTOMS, SIGNS, \& ILL-DEFINED } \\
\text { CONDITIONS }\end{array}$ & \begin{tabular}{|l|}
$780-799$ \\
\end{tabular} & 3 & 1 & 0 & 6 \\
\hline -Symptoms & 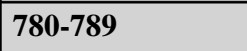 & 3 & 1 & 0 & 6 \\
\hline INJURY \& POISONING & $800-999$ & 1 & 4 & 0 & 27 \\
\hline
\end{tabular}

(Continued)

*Only those diagnostic categories and gender/job category combinations with at least one occurrence appear in this table. 
East Tennessee Technology Park 2007

Absence Data

Appendix I. Number of Diagnoses in Each Diagnostic Category by Gender and Job Category*

\begin{tabular}{|c|c|c|c|c|c|}
\hline & & \multicolumn{4}{|c|}{ Men } \\
\hline & & \multicolumn{4}{|c|}{ Job Category } \\
\hline & & Professional & $\begin{array}{c}\text { Administrative } \\
\text { Support }\end{array}$ & Technical Support & Service \\
\hline Diagnostic Category & ICD-9-CM Code & \multirow[b]{2}{*}{0} & \multirow[b]{2}{*}{1} & \multirow[b]{2}{*}{0} & \multirow[b]{2}{*}{0} \\
\hline -Fracture - Skull & $800-804$ & & & & \\
\hline -Fracture - Upper Limb & 810-819 & 0 & 3 & 0 & 0 \\
\hline -Fracture - Lower Limb & $820-829$ & 2 & 1 & 0 & 0 \\
\hline -Dislocation & 830-839 & 3 & 0 & 0 & 0 \\
\hline -Sprains \& Strains - Back & 846-847 & 1 & 0 & 0 & 0 \\
\hline -Open Wound - Lower Limb & $890-897$ & 0 & 1 & 0 & 0 \\
\hline -Contusion & $920-924$ & 0 & 0 & 0 & 1 \\
\hline -Complications \& Unspecified Injuries & 958-959 & 0 & 0 & 0 & 1 \\
\hline -Complications of Surgical/Medical Care & 996-999 & 1 & 0 & 0 & 0 \\
\hline
\end{tabular}

*Only those diagnostic categories and gender/job category combinations with at least one occurrence appear in this table. 
East Tennessee Technology Park 2007

Absence Data

Appendix I. Number of Diagnoses in Each Diagnostic Category by Gender and Job Category*

\begin{tabular}{|c|c|c|c|c|c|}
\hline & & \multicolumn{4}{|c|}{ Men } \\
\hline & & \multicolumn{3}{|c|}{ Job Category } & \multirow[b]{2}{*}{ TOTAL } \\
\hline & & Security and Fire & Crafts & Line Operators & \\
\hline Diagnostic Category & ICD-9-CM Code & \multirow[b]{2}{*}{0} & \multirow[b]{2}{*}{0} & \multirow[b]{2}{*}{0} & \multirow[b]{2}{*}{1} \\
\hline -Fracture - Skull & $800-804$ & & & & \\
\hline -Fracture - Neck, Trunk & 805-809 & 0 & 0 & 0 & 3 \\
\hline -Fracture - Upper Limb & 810-819 & 0 & 0 & 0 & 3 \\
\hline -Fracture - Lower Limb & $820-829$ & 0 & 0 & 0 & 3 \\
\hline -Dislocation & $830-839$ & 0 & 1 & 0 & 4 \\
\hline -Sprains \& Strains - Back & 846-847 & 0 & 0 & 0 & 1 \\
\hline -Sprains \& Strains - Other & $840-845,848$ & 1 & 3 & 0 & 6 \\
\hline -Internal Injury - Thorax, Abdomen, Pelvis & $860-869$ & 0 & 0 & 0 & 1 \\
\hline -Open Wound - Head, Neck, Trunk & $870-879$ & 0 & 0 & 0 & 1 \\
\hline -Open Wound - Lower Limb & 890-897 & 0 & 0 & 0 & 1 \\
\hline -Contusion & $920-924$ & 0 & 0 & 0 & 1 \\
\hline -Complications \& Unspecified Injuries & 958-959 & 0 & 0 & 0 & 1 \\
\hline -Complications of Surgical/Medical Care & 996-999 & 0 & 0 & 0 & 1 \\
\hline
\end{tabular}

\begin{tabular}{|c|c|c|c|c|c|c|c|c|}
\hline & \multicolumn{8}{|c|}{ Men } \\
\hline & \multicolumn{7}{|c|}{ Job Category } & \multirow[b]{2}{*}{ TOTAL } \\
\hline & Professional & $\begin{array}{c}\text { Administrative } \\
\text { Support }\end{array}$ & $\begin{array}{l}\text { Technical } \\
\text { Support }\end{array}$ & Service & $\begin{array}{l}\text { Security and } \\
\text { Fire }\end{array}$ & Crafts & $\begin{array}{c}\text { Line } \\
\text { Operators }\end{array}$ & \\
\hline Diagnostic Category & \multirow[b]{2}{*}{25} & \multirow[b]{2}{*}{12} & \multirow[b]{2}{*}{1} & \multirow[b]{2}{*}{18} & \multirow[b]{2}{*}{5} & \multirow[b]{2}{*}{14} & \multirow[b]{2}{*}{3} & \multirow[b]{2}{*}{78} \\
\hline Total & & & & & & & & \\
\hline
\end{tabular}

*Only those diagnostic categories and gender/job category combinations with at least one occurrence appear in this table. 
East Tennessee Technology Park 2007

Absence Data

Appendix J. Total Number of Calendar Days Absent in Each Diagnostic Category by Gender and Job Category*

\begin{tabular}{|c|c|c|c|c|}
\hline & \multicolumn{3}{|c|}{ Women } \\
\hline & & \multicolumn{3}{|c|}{ Job Category } \\
\hline & & Professional & \begin{tabular}{|c|} 
Administrative \\
Support
\end{tabular} & Technical Support \\
\hline Diagnostic Category & ICD-9-CM Code & \multirow[b]{2}{*}{0} & \multirow[b]{2}{*}{0} & \multirow[b]{2}{*}{0} \\
\hline MALIGNANT NEOPLASMS & $140-208,230-234$ & & & \\
\hline ENDOCRINE/METABOLIC/IMMUNITY & 240-279 & 18 & 0 & 0 \\
\hline NERVOUS SYSTEM (NS) \& SENSE ORGANS & 320-389 & 0 & 0 & 70 \\
\hline CIRCULATORY SYSTEM & $390-459$ & 34 & 0 & 0 \\
\hline RESPIRATORY SYSTEM & $460-519$ & 0 & 64 & 0 \\
\hline DIGESTIVE SYSTEM & $520-579$ & 10 & 39 & 0 \\
\hline GENITOURINARY SYSTEM & $580-629$ & 20 & 101 & 0 \\
\hline SKIN \& SUBCUTANEOUS TISSUE & 680-709 & 20 & 0 & 0 \\
\hline $\begin{array}{l}\text { MUSCULOSKELETAL \& CONNECTIVE } \\
\text { TISSUE }\end{array}$ & 710-739 & 7 & 127 & 31 \\
\hline CONGENITAL ANOMALIES & 740-759 & 130 & 0 & 0 \\
\hline INJURY \& POISONING & $800-999$ & 7 & 185 & 0 \\
\hline
\end{tabular}

\begin{tabular}{|c|c|c|c|c|c|}
\hline & & \multicolumn{4}{|c|}{ Women } \\
\hline & & \multicolumn{3}{|c|}{ Job Category } & \multirow[b]{2}{*}{ TOTAL } \\
\hline & & Service & Security and Fire & Line Operators & \\
\hline Diagnostic Category & ICD-9-CM Code & \multirow[b]{2}{*}{68} & \multirow[b]{2}{*}{0} & \multirow[b]{2}{*}{0} & \multirow[b]{2}{*}{68} \\
\hline MALIGNANT NEOPLASMS & $140-208,230-234$ & & & & \\
\hline ENDOCRINE/METABOLIC/IMMUNITY & 240-279 & 0 & 0 & 0 & 18 \\
\hline NERVOUS SYSTEM (NS) \& SENSE ORGANS & 320-389 & 0 & 0 & 52 & 122 \\
\hline CIRCULATORY SYSTEM & $390-459$ & 0 & 0 & 0 & 34 \\
\hline RESPIRATORY SYSTEM & $460-519$ & 0 & 0 & 0 & 64 \\
\hline DIGESTIVE SYSTEM & $520-579$ & 43 & 0 & 0 & 92 \\
\hline GENITOURINARY SYSTEM & 580-629 & 0 & 0 & 0 & 121 \\
\hline SKIN \& SUBCUTANEOUS TISSUE & $680-709$ & 0 & 0 & 0 & 20 \\
\hline $\begin{array}{l}\text { MUSCULOSKELETAL \& CONNECTIVE } \\
\text { TISSUE }\end{array}$ & 710-739 & 0 & 113 & 0 & 278 \\
\hline CONGENITAL ANOMALIES & 740-759 & 0 & 0 & 0 & 130 \\
\hline INJURY \& POISONING & $800-999$ & 0 & 0 & 0 & 192 \\
\hline
\end{tabular}

\footnotetext{
*Absences with >1 ICD-9-CM code in the same diagnostic category were counted only once. Only those diagnostic categories and gender/job category combinations with at least one occurrence appear in this table.
} 
East Tennessee Technology Park 2007

Absence Data

Appendix J. Total Number of Calendar Days Absent in Each Diagnostic Category by Gender and Job Category*

\begin{tabular}{|c|c|c|c|c|c|}
\hline & \multicolumn{4}{|c|}{ Men } \\
\hline & & \multicolumn{4}{|c|}{ Job Category } \\
\hline & & Professional & \begin{tabular}{c|} 
Administrative \\
Support
\end{tabular} & Technical Support & Service \\
\hline Diagnostic Category & ICD-9-CM Code & \multirow[b]{2}{*}{61} & \multirow[b]{2}{*}{0} & \multirow[b]{2}{*}{0} & \multirow[b]{2}{*}{0} \\
\hline MALIGNANT NEOPLASMS & $140-208,230-234$ & & & & \\
\hline ENDOCRINE/METABOLIC/IMMUNITY & $240-279$ & 21 & 0 & 0 & 0 \\
\hline MENTAL DISORDERS & $290-319$ & 0 & 0 & 0 & 0 \\
\hline NERVOUS SYSTEM (NS) \& SENSE ORGANS & $320-389$ & 45 & 0 & 0 & 1 \\
\hline CIRCULATORY SYSTEM & $390-459$ & 63 & 96 & 0 & 0 \\
\hline RESPIRATORY SYSTEM & $460-519$ & 49 & 0 & 0 & 27 \\
\hline DIGESTIVE SYSTEM & $520-579$ & 19 & 0 & 45 & 36 \\
\hline GENITOURINARY SYSTEM & 580-629 & 19 & 0 & 0 & 0 \\
\hline $\begin{array}{l}\text { MUSCULOSKELETAL \& CONNECTIVE } \\
\text { TISSUE }\end{array}$ & 710-739 & 69 & 0 & 0 & 155 \\
\hline CONGENITAL ANOMALIES & 740-759 & 0 & 0 & 0 & 0 \\
\hline $\begin{array}{l}\text { SYMPTOMS, SIGNS, \& ILL-DEFINED } \\
\text { CONDITIONS }\end{array}$ & 780-799 & 60 & 0 & 0 & 0 \\
\hline INJURY \& POISONING & $800-999$ & 320 & 167 & 0 & 25 \\
\hline
\end{tabular}

\begin{tabular}{|c|c|c|c|c|c|}
\hline & & \multicolumn{4}{|c|}{ Men } \\
\hline & & \multicolumn{3}{|c|}{ Job Category } & \multirow[b]{2}{*}{ TOTAI } \\
\hline & & Security and Fire & Crafts & Line Operators & \\
\hline Diagnostic Category & ICD-9-CM Code & \multirow[b]{2}{*}{0} & \multirow[b]{2}{*}{7} & \multirow[b]{2}{*}{18} & \multirow[b]{2}{*}{86} \\
\hline MALIGNANT NEOPLASMS & $140-208,230-234$ & & & & \\
\hline ENDOCRINE/METABOLIC/IMMUNITY & 240-279 & 0 & 0 & 0 & 21 \\
\hline MENTAL DISORDERS & $290-319$ & 0 & 10 & 0 & 10 \\
\hline NERVOUS SYSTEM (NS) \& SENSE ORGANS & 320-389 & 0 & 0 & 0 & $\overline{46}$ \\
\hline CIRCULATORY SYSTEM & $390-459$ & 0 & 42 & 0 & 201 \\
\hline RESPIRATORY SYSTEM & $460-519$ & 0 & 0 & 0 & 76 \\
\hline DIGESTIVE SYSTEM & 520-579 & 0 & 0 & 0 & 100 \\
\hline GENITOURINARY SYSTEM & 580-629 & 0 & 0 & 0 & 19 \\
\hline $\begin{array}{l}\text { MUSCULOSKELETAL \& CONNECTIVE } \\
\text { TISSUE }\end{array}$ & 710-739 & 109 & 354 & 10 & 697 \\
\hline CONGENITAL ANOMALIES & 740-759 & 0 & 0 & 82 & 82 \\
\hline $\begin{array}{l}\text { SYMPTOMS, SIGNS, \& ILL-DEFINED } \\
\text { CONDITIONS }\end{array}$ & 780-799 & 179 & 39 & 0 & 278 \\
\hline INJURY \& POISONING & $800-999$ & 88 & 353 & 0 & 953 \\
\hline
\end{tabular}

\footnotetext{
*Absences with >1 ICD-9-CM code in the same diagnostic category were counted only once. Only those diagnostic categories and gender/job category combinations with at least one occurrence appear in this table.
} 
East Tennessee Technology Park 2007

Absence Data

Appendix K. Age-Adjusted Illness and Injury Rates by Diagnostic Category*

Part 1. Men

\begin{tabular}{|c|c|c|c|c|c|}
\hline & & $\begin{array}{l}\text { Number of } \\
\text { Diagnoses }\end{array}$ & $\begin{array}{c}\text { Age-Adjusted } \\
\text { Rate per } 1,000 * *\end{array}$ & $\begin{array}{c}\text { Lower } 95 \% \\
\text { Confidence } \\
\text { Limit per 1,000 }\end{array}$ & $\begin{array}{c}\text { Upper } 95 \% \\
\text { Confidence } \\
\text { Limit per 1,000 }\end{array}$ \\
\hline Diagnostic Category & ICD-9-CM Code & & & & \\
\hline MALIGNANT NEOPLASMS & $140-208,230-234$ & 4 & 1.6 & 0.6 & 4.6 \\
\hline -Bone, Connective Tissue, Skin & $170-173,176$ & 1 & 0.3 & 0.0 & 2.4 \\
\hline -Genitourinary & 179-189 & 3 & 1.3 & 0.4 & 4.3 \\
\hline ENDOCRINE/METABOLIC/IMMUNITY & $240-279$ & 1 & 0.3 & 0.0 & 2.4 \\
\hline -Other Endocrine Gland Dis & $250-259$ & 1 & 0.3 & 0.0 & 2.4 \\
\hline MENTAL DISORDERS & 290-319 & 2 & 0.7 & 0.2 & 2.7 \\
\hline -Non-Psychotic Disorders & $300-302,306-316$ & 2 & 0.7 & 0.2 & 2.7 \\
\hline NERVOUS SYSTEM (NS) \& SENSE ORGANS & $320-389$ & 5 & 2.0 & 0.8 & 4.9 \\
\hline -Disorders of Peripheral NS & 350-359 & 1 & 0.6 & 0.1 & 4.5 \\
\hline -Disorders of Eye & $360-379$ & 3 & 1.0 & 0.3 & 3.1 \\
\hline -Dis of Ear \& Mastoid & 380-389 & 1 & 0.3 & 0.0 & 2.4 \\
\hline CIRCULATORY SYSTEM & $390-459$ & 5 & 2.6 & 1.1 & 6.6 \\
\hline -Ischemic Heart Dis & $410-414$ & 1 & 0.7 & 0.1 & 4.7 \\
\hline -Other Heart Dis & $420-429$ & 1 & 0.7 & 0.1 & 4.7 \\
\hline -Dis of Veins, Lymphatics, Other & 451-459 & 3 & 1.3 & 0.4 & 4.3 \\
\hline RESPIRATORY SYSTEM & $460-519$ & 5 & 3.3 & 1.4 & 7.9 \\
\hline -Other Dis Upper Respiratory Tract & $470-478$ & 2 & 1.3 & 0.3 & 5.3 \\
\hline -Pneumonia \& Influenza & $480-487$ & 2 & 1.3 & 0.3 & 5.2 \\
\hline -Chronic Obstructive Dis & $490-496$ & 1 & 0.7 & 0.1 & 4.7 \\
\hline DIGESTIVE SYSTEM & $520-579$ & 6 & 4.2 & 1.8 & 9.9 \\
\hline -Oral Cavity, Saliva Glands, Jaw & $520-529$ & 1 & 0.3 & 0.0 & 2.4 \\
\hline -Esophagus, Stomach, Duodenum & $\mathbf{5 3 0 - 5 3 7}$ & 1 & 1.0 & 0.1 & 7.2 \\
\hline -Appendicitis & $540-543$ & 1 & 0.6 & 0.1 & 4.5 \\
\hline -Hernia & $550-553$ & 1 & 1.0 & 0.1 & 7.2 \\
\hline -Other Intestinal Dis & $560-569$ & 2 & 1.3 & 0.3 & 5.0 \\
\hline GENITOURINARY SYSTEM & $580-629$ & 1 & 0.3 & 0.0 & 2.4 \\
\hline -Male Genital Organ Dis & $600-608$ & 1 & 0.3 & 0.0 & 2.4 \\
\hline $\begin{array}{l}\text { MUSCULOSKELETAL \& CONNECTIVE } \\
\text { TISSUE }\end{array}$ & $710-739$ & 15 & 8.7 & 5.0 & 15.3 \\
\hline -Arthropathies & $710-719$ & 7 & 5.7 & 2.7 & 12.3 \\
\hline -Dorsopathies & $720-724$ & 6 & 2.0 & 0.9 & 4.5 \\
\hline -Rheumatism, Excluding Back & 725-729 & 2 & 1.0 & 0.2 & 4.3 \\
\hline CONGENITAL ANOMALIES & \begin{tabular}{|l|}
$740-759$ \\
\end{tabular} & 1 & 0.6 & 0.1 & 4.5 \\
\hline $\begin{array}{l}\text { SYMPTOMS, SIGNS, \& ILL-DEFINED } \\
\text { CONDITIONS }\end{array}$ & 780-799 & 6 & 4.4 & 1.9 & 10.0 \\
\hline -Symptoms & 780-789 & 6 & 4.4 & 1.9 & 10.0 \\
\hline
\end{tabular}

(Continued)

*Only those diagnostic categories with at least one occurrence appear in this table.

**Standardized to age distribution of 2000 U.S. population. 
East Tennessee Technology Park 2007

Absence Data

Appendix K. Age-Adjusted Illness and Injury Rates by Diagnostic Category*

Part 1. Men

\begin{tabular}{|c|c|c|c|c|c|}
\hline & & $\begin{array}{l}\text { Number of } \\
\text { Diagnoses }\end{array}$ & $\begin{array}{c}\text { Age-Adjusted } \\
\text { Rate per 1,000** }\end{array}$ & $\begin{array}{c}\text { Lower } 95 \% \\
\text { Confidence } \\
\text { Limit per 1,000 }\end{array}$ & $\begin{array}{c}\text { Upper } 95 \% \\
\text { Confidence } \\
\text { Limit per 1,000 }\end{array}$ \\
\hline Diagnostic Category & ICD-9-CM Code & \multirow[b]{2}{*}{27} & \multirow[b]{2}{*}{18.5} & \multirow[b]{2}{*}{12.5} & \multirow[b]{2}{*}{27.4} \\
\hline INJURY \& POISONING & 800-999 & & & & \\
\hline -Fracture - Skull & 800-804 & 4 & 2.7 & 1.0 & 7.1 \\
\hline -Fracture - Upper Limb & 810-819 & 3 & 2.0 & 0.6 & 6.2 \\
\hline -Fracture - Lower Limb & $820-829$ & 3 & 1.9 & 0.6 & 6.0 \\
\hline -Dislocation & $830-839$ & 4 & 2.2 & 0.8 & 6.1 \\
\hline -Sprains \& Strains - Back & 846-847 & 1 & 0.6 & 0.1 & 4.5 \\
\hline -Sprains \& Strains - Other & $840-845,848$ & 6 & 4.9 & 2.1 & 11.5 \\
\hline -Internal Injury - Thorax, Abdomen, Pelvis & $860-869$ & 1 & 0.7 & 0.1 & 4.7 \\
\hline -Open Wound - Head, Neck, Trunk & 870-879 & 2 & 1.3 & 0.3 & 5.3 \\
\hline -Contusion & $920-924$ & 1 & 1.1 & 0.2 & 8.1 \\
\hline -Complications \& Unspecified Injuries & 958-959 & 1 & 0.3 & 0.0 & 2.4 \\
\hline -Complications of Surgical/Medical Care & 996-999 & 1 & 0.6 & 0.1 & 4.5 \\
\hline Total & & 78 & 47.4 & 37.4 & 60.1 \\
\hline
\end{tabular}

*Only those diagnostic categories with at least one occurrence appear in this table.

**Standardized to age distribution of 2000 U.S. population. 
East Tennessee Technology Park 2007

Absence Data

Appendix K. Age-Adjusted Illness and Injury Rates by Diagnostic Category*

Part 2. Women

\begin{tabular}{|c|c|c|c|c|c|}
\hline & & $\begin{array}{l}\text { Number of } \\
\text { Diagnoses }\end{array}$ & $\begin{array}{c}\text { Age-Adjusted } \\
\text { Rate per } 1,000 * *\end{array}$ & \begin{tabular}{|c|} 
Lower $95 \%$ \\
Confidence \\
Limit per 1,000 \\
\end{tabular} & $\begin{array}{c}\text { Upper } 95 \% \\
\text { Confidence } \\
\text { Limit per 1,000 }\end{array}$ \\
\hline Diagnostic Category & ICD-9-CM Code & & & & \\
\hline MALIGNANT NEOPLASMS & $140-208,230-234$ & 1 & 3.9 & 0.5 & 27.6 \\
\hline -Carcinoma in situ & $230-234$ & 1 & 3.9 & 0.5 & 27.6 \\
\hline ENDOCRINE/METABOLIC/IMMUNITY & 240-279 & 1 & 1.1 & 0.2 & 7.8 \\
\hline -Thyroid Gland Disorders & $240-246$ & 1 & 1.1 & 0.2 & 7.8 \\
\hline NERVOUS SYSTEM (NS) \& SENSE ORGANS & 320-389 & 3 & 5.9 & 1.7 & 19.7 \\
\hline -Other Disorders of Central NS & 340-349 & 1 & 3.0 & 0.4 & 21.5 \\
\hline -Disorders of Peripheral NS & 350-359 & 2 & 2.8 & 0.7 & 11.3 \\
\hline CIRCULATORY SYSTEM & 390-459 & 1 & 1.1 & 0.2 & 7.8 \\
\hline -Cerebrovascular Dis & $430-438$ & 1 & 1.1 & 0.2 & 7.8 \\
\hline RESPIRATORY SYSTEM & $460-519$ & 3 & 3.3 & 1.1 & 10.2 \\
\hline -Acute Respiratory Infections & $460-466$ & 1 & 1.1 & 0.2 & 7.8 \\
\hline -Other Dis Upper Respiratory Tract & 470-478 & 2 & 2.2 & 0.5 & 8.8 \\
\hline DIGESTIVE SYSTEM & $520-579$ & 4 & 7.8 & 2.5 & 24.2 \\
\hline -Hernia & $550-553$ & 1 & 3.9 & 0.5 & 27.6 \\
\hline -Other Digestive Dis & 570-579 & 3 & 3.9 & 1.3 & 12.3 \\
\hline GENITOURINARY SYSTEM & $580-629$ & 8 & 15.5 & 7.3 & 33.0 \\
\hline -Other Urinary Dis & 590-599 & 1 & 1.4 & 0.2 & 10.0 \\
\hline -Other Female Disorders & $617-629$ & 7 & 14.1 & 6.3 & 31.6 \\
\hline SKIN \& SUBCUTANEOUS TISSUE & 680-709 & 1 & 3.9 & 0.5 & 27.6 \\
\hline -Other & 700-709 & 1 & 3.9 & 0.5 & 27.6 \\
\hline $\begin{array}{l}\text { MUSCULOSKELETAL \& CONNECTIVE } \\
\text { TISSUE }\end{array}$ & 710-739 & 8 & 20.6 & 8.6 & 49.6 \\
\hline -Arthropathies & 710-719 & 1 & 1.4 & 0.2 & 10.0 \\
\hline -Dorsopathies & 720-724 & 2 & 4.4 & 1.0 & 19.4 \\
\hline -Rheumatism, Excluding Back & 725-729 & 4 & 7.5 & 2.4 & 23.8 \\
\hline -Other Dis \& Acquired Deformities & 730-739 & 1 & 7.3 & 1.0 & 51.5 \\
\hline CONGENITAL ANOMALIES & 740-759 & 1 & 1.4 & 0.2 & 10.0 \\
\hline INJURY \& POISONING & $800-999$ & 4 & 7.2 & 2.2 & 23.4 \\
\hline -Fracture - Lower Limb & 820-829 & 2 & 2.2 & 0.5 & 8.8 \\
\hline -Sprains \& Strains - Back & $846-847$ & 1 & 3.9 & 0.5 & 27.6 \\
\hline -Sprains \& Strains - Other & $840-845,848$ & 1 & 1.1 & 0.2 & 7.8 \\
\hline Total & & 35 & 71.6 & 48.1 & 106.6 \\
\hline
\end{tabular}

* Only those diagnostic categories with at least one occurrence appear in this table.

**Standardized to age distribution of 2000 U.S. population. 
East Tennessee Technology Park 2007

Absence Data

Appendix K. Age-Adjusted Illness and Injury Rates by Diagnostic Category*

Part 3. Men and Women

\begin{tabular}{|c|c|c|c|c|c|}
\hline & & $\begin{array}{l}\text { Number of } \\
\text { Diagnoses }\end{array}$ & $\begin{array}{c}\text { Age-Adjusted } \\
\text { Rate per } 1,000 * *\end{array}$ & $\begin{array}{c}\text { Lower } 95 \% \\
\text { Confidence } \\
\text { Limit per 1,000 }\end{array}$ & $\begin{array}{c}\text { Upper } 95 \% \\
\text { Confidence } \\
\text { Limit per 1,000 }\end{array}$ \\
\hline Diagnostic Category & ICD-9-CM Code & & & & \\
\hline MALIGNANT NEOPLASMS & $140-208,230-234$ & 5 & 1.8 & 0.7 & 4.6 \\
\hline -Bone, Connective Tissue, Skin & 170-173, 176 & 1 & 0.3 & 0.0 & 1.8 \\
\hline -Genitourinary & 179-189 & 3 & 1.0 & 0.3 & 3.1 \\
\hline -Carcinoma in situ & $230-234$ & 1 & 0.6 & 0.1 & 4.2 \\
\hline ENDOCRINE/METABOLIC/IMMUNITY & $240-279$ & 2 & 0.5 & 0.1 & 2.1 \\
\hline -Thyroid Gland Disorders & $240-246$ & 1 & 0.3 & 0.0 & 1.8 \\
\hline -Other Endocrine Gland Dis & $250-259$ & 1 & 0.3 & 0.0 & 1.8 \\
\hline MENTAL DISORDERS & $290-319$ & 2 & 0.5 & 0.1 & 2.1 \\
\hline -Non-Psychotic Disorders & $300-302,306-316$ & 2 & 0.5 & 0.1 & 2.1 \\
\hline NERVOUS SYSTEM (NS) \& SENSE ORGANS & 320-389 & 8 & 3.1 & 1.5 & 6.6 \\
\hline -Other Disorders of Central NS & $340-349$ & 1 & 0.8 & 0.1 & 5.3 \\
\hline -Disorders of Peripheral NS & $350-359$ & 3 & 1.3 & 0.4 & 4.1 \\
\hline -Disorders of Eye & $360-379$ & 3 & 0.8 & 0.3 & 2.4 \\
\hline -Dis of Ear \& Mastoid & $380-389$ & 1 & 0.3 & 0.0 & 1.8 \\
\hline CIRCULATORY SYSTEM & $390-459$ & 6 & 2.4 & 1.0 & 5.6 \\
\hline -Ischemic Heart Dis & $410-414$ & 1 & 0.6 & 0.1 & 4.2 \\
\hline -Other Heart Dis & $420-429$ & 1 & 0.6 & 0.1 & 4.2 \\
\hline -Cerebrovascular Dis & $430-438$ & 1 & 0.3 & 0.0 & 1.8 \\
\hline -Dis of Veins, Lymphatics, Other & 451-459 & 3 & 1.0 & 0.3 & 3.1 \\
\hline RESPIRATORY SYSTEM & $460-519$ & 8 & 3.6 & 1.7 & 7.5 \\
\hline -Acute Respiratory Infections & $460-466$ & 1 & 0.3 & 0.0 & 1.8 \\
\hline -Other Dis Upper Respiratory Tract & $470-478$ & 4 & 1.7 & 0.6 & 4.9 \\
\hline -Pneumonia \& Influenza & $480-487$ & 2 & 1.0 & 0.3 & 4.2 \\
\hline -Chronic Obstructive Dis & $490-496$ & 1 & 0.6 & 0.1 & 4.2 \\
\hline DIGESTIVE SYSTEM & $520-579$ & 10 & 4.8 & 2.5 & 9.2 \\
\hline -Oral Cavity, Saliva Glands, Jaw & $520-529$ & 1 & 0.3 & 0.0 & 1.8 \\
\hline -Esophagus, Stomach, Duodenum & $530-537$ & 1 & 0.8 & 0.1 & 5.3 \\
\hline -Appendicitis & $540-543$ & 1 & 0.4 & 0.1 & 3.1 \\
\hline -Hernia & $550-553$ & 2 & 1.3 & 0.3 & 5.4 \\
\hline -Other Intestinal Dis & $560-569$ & 2 & 0.9 & 0.2 & 3.5 \\
\hline -Other Digestive Dis & $570-579$ & 3 & 1.1 & 0.4 & 3.6 \\
\hline GENITOURINARY SYSTEM & $580-629$ & 9 & 4.3 & 2.1 & 8.8 \\
\hline -Other Urinary Dis & $590-599$ & 1 & 0.4 & 0.1 & 3.1 \\
\hline -Male Genital Organ Dis & $600-608$ & 1 & 0.3 & 0.0 & $\overline{1.8}$ \\
\hline -Other Female Disorders & $617-629$ & 7 & 3.6 & 1.6 & 8.1 \\
\hline
\end{tabular}

(Continued)

* Only those diagnostic categories with at least one occurrence appear in this table.

**Standardized to age distribution of 2000 U.S. population. 
East Tennessee Technology Park 2007

Absence Data

Appendix K. Age-Adjusted Illness and Injury Rates by Diagnostic Category*

Part 3. Men and Women

\begin{tabular}{|c|c|c|c|c|c|}
\hline & & $\begin{array}{c}\text { Number of } \\
\text { Diagnoses }\end{array}$ & $\begin{array}{c}\text { Age-Adjusted } \\
\text { Rate per } 1,000 * *\end{array}$ & $\begin{array}{c}\text { Lower } 95 \% \\
\text { Confidence } \\
\text { Limit per 1,000 }\end{array}$ & $\begin{array}{c}\text { Upper } 95 \% \\
\text { Confidence } \\
\text { Limit per 1,000 }\end{array}$ \\
\hline Diagnostic Category & ICD-9-CM Code & & & & \\
\hline SKIN \& SUBCUTANEOUS TISSUE & 680-709 & 1 & 0.6 & 0.1 & 4.2 \\
\hline -Other & 700-709 & 1 & 0.6 & 0.1 & 4.2 \\
\hline $\begin{array}{l}\text { MUSCULOSKELETAL \& CONNECTIVE } \\
\text { TISSUE }\end{array}$ & 710-739 & 23 & 10.9 & 6.9 & 17.2 \\
\hline -Arthropathies & 710-719 & 8 & 4.8 & 2.3 & 10.0 \\
\hline -Dorsopathies & $720-724$ & 8 & 2.7 & 1.3 & 5.9 \\
\hline -Rheumatism, Excluding Back & 725-729 & 6 & 2.4 & 1.0 & 5.6 \\
\hline -Other Dis \& Acquired Deformities & 730-739 & 1 & 1.0 & 0.1 & 6.9 \\
\hline CONGENITAL ANOMALIES & 740-759 & 2 & 0.9 & 0.2 & 3.5 \\
\hline $\begin{array}{l}\text { SYMPTOMS, SIGNS, \& ILL-DEFINED } \\
\text { CONDITIONS }\end{array}$ & 780-799 & 6 & 3.5 & 1.5 & 8.0 \\
\hline -Symptoms & 780-789 & 6 & 3.5 & 1.5 & 8.0 \\
\hline INJURY \& POISONING & 800-999 & 31 & 16.3 & 11.2 & 23.7 \\
\hline -Fracture - Skull & $800-804$ & 4 & 2.4 & 0.9 & 6.3 \\
\hline -Fracture - Upper Limb & 810-819 & 3 & 1.8 & 0.6 & 5.5 \\
\hline -Fracture - Lower Limb & 820-829 & 5 & 2.0 & 0.8 & 5.0 \\
\hline -Dislocation & 830-839 & 4 & 1.6 & 0.6 & 4.3 \\
\hline -Sprains \& Strains - Back & $846-847$ & 2 & 1.0 & 0.3 & 4.2 \\
\hline -Sprains \& Strains - Other & $840-845,848$ & 7 & 4.1 & 1.8 & 9.3 \\
\hline -Internal Injury - Thorax, Abdomen, Pelvis & $860-869$ & 1 & 0.6 & 0.1 & 4.2 \\
\hline -Open Wound - Head, Neck, Trunk & 870-879 & 2 & 1.2 & 0.3 & 4.7 \\
\hline -Contusion & $920-924$ & 1 & 1.0 & 0.1 & 6.9 \\
\hline -Complications \& Unspecified Injuries & 958-959 & 1 & 0.3 & 0.0 & 1.8 \\
\hline -Complications of Surgical/Medical Care & 996-999 & 1 & 0.4 & 0.1 & 3.1 \\
\hline Total & & 113 & 53.2 & 43.6 & 65.0 \\
\hline
\end{tabular}

* Only those diagnostic categories with at least one occurrence appear in this table.

**Standardized to age distribution of 2000 U.S. population. 
East Tennessee Technology Park 2007

OSHA Data

Appendix L. Number of Workers with at Least One OSHA Event by Gender, Age, and Job Category*

\begin{tabular}{|c|c|c|c|c|c|c|c|c|c|c|c|}
\hline \multirow{3}{*}{ Job Category } & \multicolumn{5}{|c|}{ Women } & \multicolumn{5}{|c|}{ Men } & \multirow[b]{3}{*}{ TOTAL } \\
\hline & \multicolumn{4}{|c|}{ Age Group } & \multirow[b]{2}{*}{ TOTAL } & \multicolumn{4}{|c|}{ Age Group } & \multirow[b]{2}{*}{ TOTAL } & \\
\hline & $16-29$ & 30 - 39 & $40-49$ & $50+$ & & $16-29$ & $30-39$ & $40-49$ & $50+$ & & \\
\hline Administrative Support & 0 & 0 & 0 & 0 & 0 & 0 & 0 & 1 & 0 & 1 & 1 \\
\hline Service & 2 & 1 & 2 & 1 & 6 & 3 & 2 & 3 & 2 & 10 & 16 \\
\hline Security and Fire & 0 & 0 & 0 & 0 & 0 & 0 & 0 & 1 & 1 & 2 & 2 \\
\hline Crafts & 0 & 0 & 0 & 0 & 0 & 0 & 1 & 3 & 2 & 6 & 6 \\
\hline Line Operators & 0 & 0 & 0 & 0 & 0 & 0 & 0 & 1 & 0 & 1 & 1 \\
\hline TOTAL & 2 & 1 & 2 & 1 & 6 & 3 & 3 & 9 & 5 & 20 & 26 \\
\hline
\end{tabular}

*Only those job categories and gender/age combinations with at least one OSHA event appear in this table.

Appendix M. Total Number of Workdays Lost or with Restricted Activity from OSHA Events by Gender and Age

\begin{tabular}{|c|c|c|c|c|c|c|c|c|c|}
\hline \multirow[b]{2}{*}{ Age Group } & \multicolumn{3}{|c|}{ Women } & \multicolumn{3}{|c|}{ Men } & \multicolumn{3}{|c|}{ TOTAL } \\
\hline & $\begin{array}{c}\text { Number of } \\
\text { Events }\end{array}$ & $\begin{array}{c}\text { Days } \\
\text { Restricted }\end{array}$ & Days Lost & $\begin{array}{l}\text { Number of } \\
\text { Events }\end{array}$ & $\begin{array}{c}\text { Days } \\
\text { Restricted }\end{array}$ & Days Lost & $\begin{array}{c}\text { Number of } \\
\text { Events }\end{array}$ & $\begin{array}{c}\text { Days } \\
\text { Restricted }\end{array}$ & Days Lost \\
\hline $16-29$ & 2 & 0 & 0 & 3 & 0 & 180 & 5 & 0 & 180 \\
\hline $30-39$ & 1 & 0 & 0 & 4 & 11 & 0 & 5 & 11 & 0 \\
\hline $40-49$ & 2 & 94 & 0 & 9 & 361 & 178 & 11 & 455 & 178 \\
\hline $50+$ & 1 & 0 & 0 & 6 & 232 & 80 & 7 & 232 & 80 \\
\hline TOTAL & 6 & 94 & 0 & 22 & 604 & 438 & 28 & 698 & 438 \\
\hline
\end{tabular}

Appendix N. Total Number of Workdays Lost or with Restricted Activity from OSHA Events by Gender and Job Category*

\begin{tabular}{|c|c|c|c|c|c|c|c|c|c|}
\hline \multirow[b]{2}{*}{ Job Category } & \multicolumn{3}{|c|}{ Women } & \multicolumn{3}{|c|}{ Men } & \multicolumn{3}{|c|}{ TOTAL } \\
\hline & $\begin{array}{l}\text { Number of } \\
\text { Events }\end{array}$ & $\begin{array}{c}\text { Days } \\
\text { Restricted }\end{array}$ & Days Lost & \begin{tabular}{|c|}
$\begin{array}{c}\text { Number of } \\
\text { Events }\end{array}$ \\
\end{tabular} & $\begin{array}{c}\text { Days } \\
\text { Restricted }\end{array}$ & Days Lost & $\begin{array}{c}\text { Number of } \\
\text { Events }\end{array}$ & $\begin{array}{c}\text { Days } \\
\text { Restricted }\end{array}$ & Days Lost \\
\hline Service & 6 & 94 & 0 & 12 & 184 & 268 & 18 & 278 & 268 \\
\hline Line Operators & 0 & 0 & 0 & 1 & 0 & 166 & 1 & 0 & 166 \\
\hline TOTAL & 6 & 94 & 0 & 22 & 604 & 438 & 28 & 698 & 438 \\
\hline
\end{tabular}

*Only those job categories with at least one OSHA event appear in this table. 
East Tennessee Technology Park 2007

OSHA Data

Appendix O. Number of Diagnoses in Each Diagnostic Category by Gender and Age*

\begin{tabular}{|c|c|c|c|c|c|c|}
\hline & & \multicolumn{5}{|c|}{ Women } \\
\hline & & \multicolumn{4}{|c|}{ Age Group } & \multirow[b]{2}{*}{ TOTAL } \\
\hline & & $16-29$ & $30-39$ & $40-49$ & $50+$ & \\
\hline Diagnostic Category & ICD-9-CM code & \multirow[b]{2}{*}{0} & \multirow[b]{2}{*}{0} & \multirow[b]{2}{*}{0} & \multirow[b]{2}{*}{0} & \multirow[b]{2}{*}{0} \\
\hline NERVOUS SYSTEM (NS) \& SENSE ORGANS & 320-389 & & & & & \\
\hline -Disorders of Peripheral NS & $350-359$ & 0 & 0 & 0 & 0 & 0 \\
\hline INJURY \& POISONING & 800-999 & 2 & 1 & 2 & 1 & 6 \\
\hline -Fracture - Upper Limb & 810-819 & 0 & 0 & 0 & 0 & 0 \\
\hline -Fracture - Lower Limb & $820-829$ & 0 & 0 & 0 & 0 & 0 \\
\hline -Sprains \& Strains - Back & 846-847 & 0 & 0 & 0 & 0 & 0 \\
\hline -Sprains \& Strains - Other & $840-845,848$ & 1 & 1 & 2 & 0 & 4 \\
\hline -Open Wound - Upper Limb & 880-887 & 0 & 0 & 0 & 1 & 1 \\
\hline -Open Wound - Lower Limb & 890-897 & 1 & 0 & 0 & 0 & 1 \\
\hline -Contusion & 920-924 & 0 & 0 & 0 & 0 & 0 \\
\hline -Burns & 940-949 & 0 & 0 & 0 & 0 & 0 \\
\hline
\end{tabular}

\begin{tabular}{|c|c|c|c|c|c|c|c|}
\hline & & \multicolumn{5}{|c|}{ Men } & \multirow[b]{3}{*}{ TOTAI } \\
\hline & & \multicolumn{4}{|c|}{ Age Group } & \multirow[b]{2}{*}{ TOTAL } & \\
\hline & & $16-29$ & 30 - 39 & $40-49$ & $50+$ & & \\
\hline Diagnostic Category & ICD-9-CM code & & & & & & \\
\hline NERVOUS SYSTEM (NS) \& SENSE ORGANS & 320-389 & 0 & 0 & 1 & 1 & 2 & 2 \\
\hline -Disorders of Peripheral NS & 350-359 & 0 & 0 & 1 & 1 & 2 & 2 \\
\hline INJURY \& POISONING & 800-999 & 3 & 4 & 9 & 5 & 21 & 27 \\
\hline -Fracture - Upper Limb & 810-819 & 0 & 1 & 2 & 0 & 3 & 3 \\
\hline -Fracture - Lower Limb & $820-829$ & 0 & 0 & 1 & $\overline{0}$ & 1 & 1 \\
\hline -Sprains \& Strains - Back & 846-847 & 1 & 1 & 0 & 1 & 3 & 3 \\
\hline -Sprains \& Strains - Other & $840-845,848$ & 0 & 0 & 2 & 3 & 5 & 9 \\
\hline -Open Wound - Upper Limb & 880-887 & 1 & 1 & 2 & 0 & 4 & 5 \\
\hline -Open Wound - Lower Limb & 890-897 & 0 & 1 & 0 & 0 & 1 & 2 \\
\hline -Contusion & $920-924$ & 0 & 0 & 2 & 1 & 3 & 3 \\
\hline -Burns & 940-949 & 1 & 0 & 0 & 0 & 1 & 1 \\
\hline
\end{tabular}

\begin{tabular}{|c|c|c|c|c|c|c|c|c|c|c|c|}
\hline & \multicolumn{5}{|c|}{ Women } & \multicolumn{5}{|c|}{ Men } & \multirow[b]{3}{*}{ TOTAL } \\
\hline & \multicolumn{4}{|c|}{ Age Group } & \multirow[b]{2}{*}{ TOTAL } & \multicolumn{4}{|c|}{ Age Group } & \multirow[b]{2}{*}{ TOTAL } & \\
\hline & $16-29$ & $30-39$ & $40-49$ & $50+$ & & $16-29$ & $30-39$ & $40-49$ & $50+$ & & \\
\hline Diagnostic Category & & & & & & & & & & & \\
\hline Total & 2 & 1 & 2 & 1 & 6 & 3 & 4 & 10 & 6 & 23 & 29 \\
\hline
\end{tabular}

*Only those diagnostic categories and gender/age combinations with at least one OSHA event appear in this table. 
East Tennessee Technology Park 2007

OSHA Data

Appendix P. Number of Workdays Lost or with Restricted Activity in Each Diagnostic Category by Gender and Age*

\begin{tabular}{|c|c|c|c|c|c|c|c|c|c|}
\hline & & \multicolumn{8}{|c|}{ Women } \\
\hline & & \multicolumn{8}{|c|}{ Age Group } \\
\hline & & \multicolumn{2}{|c|}{$16-29$} & \multicolumn{2}{|c|}{30 - 39} & \multicolumn{2}{|c|}{$40-49$} & \multicolumn{2}{|c|}{$50+$} \\
\hline & & $\begin{array}{c}\text { Days } \\
\text { Restricted }\end{array}$ & $\begin{array}{l}\text { Days } \\
\text { Lost }\end{array}$ & $\begin{array}{c}\text { Days } \\
\text { Restricted }\end{array}$ & $\begin{array}{l}\text { Days } \\
\text { Lost }\end{array}$ & $\begin{array}{c}\text { Days } \\
\text { Restricted }\end{array}$ & $\begin{array}{l}\text { Days } \\
\text { Lost }\end{array}$ & $\begin{array}{c}\text { Days } \\
\text { Restricted }\end{array}$ & $\begin{array}{l}\text { Days } \\
\text { Lost }\end{array}$ \\
\hline Diagnostic Category & ICD-9-CM Codes & & & & & & & & \\
\hline -Sprains \& Strains - Other & $840-845,848$ & 0 & 0 & 0 & 0 & 94 & 0 & 0 & 0 \\
\hline -Open Wound - Upper Limb & $880-887$ & 0 & 0 & 0 & 0 & 0 & 0 & 0 & 0 \\
\hline -Open Wound - Lower Limb & $890-897$ & 0 & 0 & 0 & 0 & 0 & 0 & 0 & 0 \\
\hline
\end{tabular}

\begin{tabular}{|c|c|c|c|c|c|c|c|c|c|}
\hline & \multicolumn{8}{|c|}{ Men } \\
\hline & & \multicolumn{8}{|c|}{ Age Group } \\
\hline & & \multicolumn{2}{|c|}{$16-29$} & \multicolumn{2}{|c|}{30 - 39} & \multicolumn{2}{|c|}{$40-49$} & \multicolumn{2}{|c|}{$50+$} \\
\hline & & \begin{tabular}{|c|} 
Days \\
Restricted
\end{tabular} & $\begin{array}{l}\text { Days } \\
\text { Lost }\end{array}$ & $\begin{array}{c}\text { Days } \\
\text { Restricted }\end{array}$ & $\begin{array}{l}\text { Days } \\
\text { Lost }\end{array}$ & \begin{tabular}{c|} 
Days \\
Restricted
\end{tabular} & $\begin{array}{l}\text { Days } \\
\text { Lost }\end{array}$ & \begin{tabular}{c|} 
Days \\
Restricted
\end{tabular} & $\begin{array}{l}\text { Days } \\
\text { Lost }\end{array}$ \\
\hline Diagnostic Category & ICD-9-CM Codes & & & & & & & & \\
\hline -Disorders of Peripheral NS & 350-359 & 0 & 0 & 0 & 0 & 172 & 8 & 50 & 0 \\
\hline -Fracture - Upper Limb & $810-819$ & 0 & 0 & 0 & 0 & 96 & 0 & 0 & 0 \\
\hline -Fracture - Lower Limb & $820-829$ & 0 & 0 & 0 & 0 & 35 & 4 & 0 & 0 \\
\hline -Sprains \& Strains - Back & 846-847 & 0 & 180 & 3 & 0 & 0 & 0 & 180 & 0 \\
\hline -Sprains \& Strains - Other & $840-845,848$ & 0 & 0 & 0 & 0 & 0 & 166 & 2 & 80 \\
\hline -Open Wound - Upper Limb & $\begin{array}{ll}880-887 \\
\end{array}$ & 0 & 0 & 6 & 0 & 99 & 0 & 0 & 0 \\
\hline -Open Wound - Lower Limb & 890-897 & 0 & 0 & 2 & 0 & 0 & 0 & 0 & 0 \\
\hline -Contusion & 920-924 & 0 & 0 & 0 & 0 & 55 & 0 & 0 & 0 \\
\hline -Burns & $940-949$ & 0 & 0 & 0 & 0 & 0 & 0 & 0 & 0 \\
\hline
\end{tabular}

*OSHA events with >1 ICD-9-CM code in the same diagnostic category were counted only once. Only those diagnostic categories and gender/age combinations with at least one occurrence appear in this table. 
East Tennessee Technology Park 2007

OSHA Data

Appendix Q. Number of Occurrences in Each Accident Category by Gender and Age*

\begin{tabular}{|c|c|c|c|c|c|c|}
\hline & & \multicolumn{5}{|c|}{ Women } \\
\hline & & \multicolumn{4}{|c|}{ Age Group } & \multirow[b]{2}{*}{ TOTAL } \\
\hline & & $16-29$ & 30 - 39 & $40-49$ & $50+$ & \\
\hline Type of Accident & E Codes & \multirow[b]{2}{*}{0} & \multirow[b]{2}{*}{1} & \multirow[b]{2}{*}{0} & \multirow[b]{2}{*}{0} & \multirow[b]{2}{*}{1} \\
\hline Falls & E880-E888 & & & & & \\
\hline Other Accidents & E916-E928 & 1 & 0 & 1 & 1 & 3 \\
\hline
\end{tabular}

\begin{tabular}{|c|c|c|c|c|c|c|c|}
\hline & & \multicolumn{5}{|c|}{ Men } & \multirow[b]{3}{*}{ TOTAL } \\
\hline & & \multicolumn{4}{|c|}{ Age Group } & \multirow[b]{2}{*}{ TOTAL } & \\
\hline & & $16-29$ & $30-39$ & $40-49$ & $50+$ & & \\
\hline Type of Accident & E Codes & & & & & & \\
\hline Falls & E880-E888 & 0 & 0 & 0 & 1 & 1 & 2 \\
\hline Other Accidents & E916-E928 & 2 & 1 & 1 & 1 & 5 & 8 \\
\hline
\end{tabular}

*Only those accident types and gender/age combinations with at least one occurrence appear in this table. 
East Tennessee Technology Park 2007

OSHA Data

Appendix R. Number of Workdays Lost or with Restricted Activity in Each Accident Category by Gender and Age*

\begin{tabular}{|c|c|c|c|c|c|c|c|c|c|}
\hline & & \multicolumn{8}{|c|}{ Women } \\
\hline & & \multicolumn{8}{|c|}{ Age Group } \\
\hline & & \multicolumn{2}{|c|}{16 - 29} & \multicolumn{2}{|c|}{30 - 39} & \multicolumn{2}{|c|}{$40-49$} & \multicolumn{2}{|c|}{$50+$} \\
\hline & & $\begin{array}{c}\text { Days } \\
\text { Restricted }\end{array}$ & $\begin{array}{l}\text { Days } \\
\text { Lost }\end{array}$ & $\begin{array}{c}\text { Days } \\
\text { Restricted }\end{array}$ & $\begin{array}{l}\text { Days } \\
\text { Lost }\end{array}$ & $\begin{array}{c}\text { Days } \\
\text { Restricted }\end{array}$ & $\begin{array}{l}\text { Days } \\
\text { Lost }\end{array}$ & $\begin{array}{c}\text { Days } \\
\text { Restricted }\end{array}$ & $\begin{array}{l}\text { Days } \\
\text { Lost }\end{array}$ \\
\hline Type of Accident & E Codes & \multirow[b]{2}{*}{0} & \multirow[b]{2}{*}{0} & \multirow[b]{2}{*}{0} & \multirow[b]{2}{*}{0} & \multirow[b]{2}{*}{0} & \multirow[b]{2}{*}{0} & \multirow[b]{2}{*}{0} & \multirow[b]{2}{*}{0} \\
\hline Falls & \begin{tabular}{|l|} 
E880-E888 \\
\end{tabular} & & & & & & & & \\
\hline Other Accidents & E916-E928 & 0 & 0 & 0 & 0 & 94 & 0 & 0 & 0 \\
\hline
\end{tabular}

\begin{tabular}{|c|c|c|c|c|c|c|c|c|c|}
\hline & & \multicolumn{8}{|c|}{ Men } \\
\hline & & \multicolumn{8}{|c|}{ Age Group } \\
\hline & & \multicolumn{2}{|c|}{$16-29$} & \multicolumn{2}{|c|}{$30-39$} & \multicolumn{2}{|c|}{$40-49$} & \multicolumn{2}{|c|}{$50+$} \\
\hline & & $\begin{array}{c}\text { Days } \\
\text { Restricted }\end{array}$ & $\begin{array}{l}\text { Days } \\
\text { Lost }\end{array}$ & $\begin{array}{c}\text { Days } \\
\text { Restricted }\end{array}$ & $\begin{array}{l}\text { Days } \\
\text { Lost }\end{array}$ & $\begin{array}{c}\text { Days } \\
\text { Restricted }\end{array}$ & $\begin{array}{l}\text { Days } \\
\text { Lost }\end{array}$ & $\begin{array}{c}\text { Days } \\
\text { Restricted }\end{array}$ & $\begin{array}{l}\text { Days } \\
\text { Lost }\end{array}$ \\
\hline Type of Accident & E Codes & \multirow[b]{2}{*}{0} & \multirow[b]{2}{*}{0} & \multirow[b]{2}{*}{0} & \multirow[b]{2}{*}{0} & \multirow[b]{2}{*}{0} & \multirow[b]{2}{*}{0} & \multirow[b]{2}{*}{0} & \multirow[b]{2}{*}{0} \\
\hline Falls & E880-E888 & & & & & & & & \\
\hline Other Accidents & E916-E928 & 0 & 180 & 3 & 0 & 0 & 166 & 2 & 0 \\
\hline
\end{tabular}

*OSHA events with $>1 \mathrm{E}$ code in the same accident type were counted only once. Only those accident types and gender/age combinations with at least one occurrence appear in this table. 
East Tennessee Technology Park 2007

OSHA Data

Appendix S. Number of Diagnoses in Each Diagnostic Category by Gender and Job Category*

\begin{tabular}{|l|l|r|r|}
\hline \multicolumn{2}{|c|}{} & \multicolumn{2}{c|}{ Women } \\
\cline { 3 - 4 } \multicolumn{2}{|c|}{} & Job Category & \\
\cline { 3 - 4 } \multicolumn{2}{|c|}{ Service } & TOTAL \\
\hline Diagnostic Category & ICD-9-CM Code & & \\
\hline INJURY \& POISONING & $\mathbf{8 0 0 - 9 9 9}$ & 6 & 6 \\
\hline $\begin{array}{l}\text {-Sprains \& Strains - } \\
\text { Other }\end{array}$ & $\mathbf{8 4 0 - 8 4 5 , 8 4 8}$ & & 4 \\
\hline $\begin{array}{l}\text {-Open Wound - Upper } \\
\text { Limb }\end{array}$ & $\mathbf{8 8 0 - 8 8 7}$ & 1 & 1 \\
\hline $\begin{array}{l}\text {-Open Wound - Lower } \\
\text { Limb }\end{array}$ & $\mathbf{8 9 0 - 8 9 7}$ & 1 & 1 \\
\hline
\end{tabular}

\begin{tabular}{|l|r|r|}
\hline \multirow{4}{*}{} & \multicolumn{2}{|c|}{ Women } \\
\cline { 2 - 2 } & Job Category & \\
\cline { 2 - 2 } & Service & TOTAL \\
\hline Diagnostic Category & & \\
\cline { 1 - 1 } Total & & \\
\hline
\end{tabular}

\begin{tabular}{|c|c|c|c|c|c|c|c|}
\hline & & \multicolumn{6}{|c|}{ Men } \\
\hline & & \multicolumn{5}{|c|}{ Job Category } & \multirow[b]{2}{*}{ TOTAL } \\
\hline & & $\begin{array}{l}\text { Administrative } \\
\text { Support }\end{array}$ & Service & Security and Fire & Crafts & Line Operators & \\
\hline Diagnostic Category & ICD-9-CM Code & & & & & & \\
\hline $\begin{array}{l}\text { NERVOUS SYSTEM } \\
\text { (NS) \& SENSE ORGANS }\end{array}$ & 320-389 & 0 & 1 & 0 & 1 & 0 & 2 \\
\hline $\begin{array}{l}\text {-Disorders of Peripheral } \\
\text { NS }\end{array}$ & 350-359 & 0 & 1 & 0 & 1 & 0 & 2 \\
\hline INJURY \& POISONING & $800-999$ & 1 & 11 & 2 & 6 & 1 & 21 \\
\hline -Fracture - Upper Limb & 810-819 & 0 & 1 & 1 & 1 & 0 & 3 \\
\hline -Fracture - Lower Limb & $820-829$ & 0 & 0 & 0 & 1 & 0 & 1 \\
\hline -Sprains \& Strains - Back & $846-847$ & 0 & 2 & 0 & 1 & 0 & 3 \\
\hline $\begin{array}{l}\text {-Sprains \& Strains - } \\
\text { Other }\end{array}$ & $840-845,848$ & 1 & 2 & 1 & 0 & 1 & 5 \\
\hline $\begin{array}{l}\text {-Open Wound - Upper } \\
\text { Limb }\end{array}$ & 880-887 & 0 & 3 & 0 & 1 & 0 & 4 \\
\hline $\begin{array}{l}\text {-Open Wound - Lower } \\
\text { Limb }\end{array}$ & 890-897 & 0 & 0 & 0 & 1 & 0 & 1 \\
\hline -Contusion & 920-924 & 0 & 2 & 0 & 1 & 0 & 3 \\
\hline -Burns & 940-949 & 0 & 1 & 0 & 0 & 0 & 1 \\
\hline
\end{tabular}

*Only those diagnostic categories and gender/job category combinations with at least one occurrence appear in this table. 
East Tennessee Technology Park 2007

OSHA Data

Appendix S. Number of Diagnoses in Each Diagnostic Category by Gender and Job Category*

\begin{tabular}{|l|r|r|r|r|r|r|}
\hline \multirow{4}{*}{} & \multicolumn{7}{|c|}{ Men } & \\
\cline { 2 - 8 } & \multicolumn{7}{|c|}{ Job Category } \\
\cline { 2 - 8 } & $\begin{array}{c}\text { Administrative } \\
\text { Support }\end{array}$ & Service & Security and Fire & Crafts & Line Operators & TOTAL \\
\hline Diagnostic Category & 1 & 12 & & & & \\
\cline { 1 - 7 } Total & & & & & & 23 \\
\hline
\end{tabular}

*Only those diagnostic categories and gender/job category combinations with at least one occurrence appear in this table. 
East Tennessee Technology Park 2007

OSHA Data

Appendix T. Number of Workdays Lost or with Restricted Activity in Each Diagnostic Category by Gender and Job Category*

\begin{tabular}{|c|c|c|c|}
\hline & \multirow{2}{*}{\multicolumn{2}{|c|}{$\frac{\text { Women }}{\text { Job Category }}$}} \\
\hline & & & \\
\hline & & \multicolumn{2}{|c|}{ Service } \\
\hline & & $\begin{array}{c}\text { Days } \\
\text { Restricted }\end{array}$ & Days Lost \\
\hline Diagnostic Category & ICD-9-CM Codes & \multirow[b]{2}{*}{94} & \multirow[b]{2}{*}{0} \\
\hline -Sprains \& Strains - Other & $840-845,848$ & & \\
\hline -Open Wound - Upper Limb & 880-887 & 0 & 0 \\
\hline -Open Wound - Lower Limb & 890-897 & 0 & 0 \\
\hline
\end{tabular}

*OSHA events with >1 ICD-9-CM code in the same diagnostic category were counted only once. Only those diagnostic categories and gender/job category combinations with at least one occurrence appear in this table. 
East Tennessee Technology Park 2007

OSHA Data

Appendix T. Number of Workdays Lost or with Restricted Activity in Each Diagnostic Category by Gender and Job Category*

\begin{tabular}{|c|c|c|c|c|c|c|c|}
\hline & & \multicolumn{6}{|c|}{ Men } \\
\hline & & \multicolumn{6}{|c|}{ Job Category } \\
\hline & & \multicolumn{2}{|c|}{$\begin{array}{c}\text { Administrative } \\
\text { Support }\end{array}$} & \multicolumn{2}{|c|}{ Service } & \multicolumn{2}{|c|}{ Security and Fire } \\
\hline & & $\begin{array}{c}\text { Days } \\
\text { Restricted }\end{array}$ & Days Lost & \begin{tabular}{|c|} 
Days \\
Restricted
\end{tabular} & Days Lost & \begin{tabular}{|c|} 
Days \\
Restricted
\end{tabular} & Days Lost \\
\hline Diagnostic Category & ICD-9-CM Codes & \multirow[b]{2}{*}{0} & \multirow[b]{2}{*}{0} & \multirow[b]{2}{*}{172} & \multirow[b]{2}{*}{8} & \multirow[b]{2}{*}{0} & \multirow[b]{2}{*}{0} \\
\hline -Disorders of Peripheral NS & $350-359$ & & & & & & \\
\hline -Fracture - Upper Limb & $810-819$ & 0 & 0 & 0 & 0 & 0 & 0 \\
\hline -Fracture - Lower Limb & $820-829$ & 0 & 0 & 0 & 0 & 0 & 0 \\
\hline -Sprains \& Strains - Back & \begin{tabular}{|l|}
$846-847$ \\
\end{tabular} & 0 & 0 & 3 & 180 & 0 & 0 \\
\hline -Sprains \& Strains - Other & $840-845,848$ & 0 & 0 & 0 & 80 & 2 & 0 \\
\hline -Open Wound - Upper Limb & $\mathbf{8 8 0 - 8 8 7}$ & 0 & 0 & 9 & 0 & 0 & 0 \\
\hline -Open Wound - Lower Limb & $890-897$ & 0 & 0 & 0 & 0 & 0 & 0 \\
\hline -Contusion & 920-924 & 0 & 0 & 0 & 0 & 0 & 0 \\
\hline -Burns & $940-949$ & 0 & 0 & 0 & 0 & 0 & 0 \\
\hline
\end{tabular}

\begin{tabular}{|c|c|c|c|c|c|}
\hline & & \multicolumn{4}{|c|}{ Men } \\
\hline & & \multicolumn{4}{|c|}{ Job Category } \\
\hline & & \multicolumn{2}{|c|}{ Crafts } & \multicolumn{2}{|c|}{ Line Operators } \\
\hline & & $\begin{array}{c}\text { Days } \\
\text { Restricted }\end{array}$ & Days Lost & $\begin{array}{c}\text { Days } \\
\text { Restricted }\end{array}$ & Days Lost \\
\hline Diagnostic Category & ICD-9-CM Codes & \multirow[b]{2}{*}{50} & \multirow[b]{2}{*}{0} & \multirow[b]{2}{*}{0} & \multirow[b]{2}{*}{0} \\
\hline -Disorders of Peripheral NS & $350-359$ & & & & \\
\hline -Fracture - Upper Limb & $810-819$ & 96 & 0 & 0 & 0 \\
\hline -Fracture - Lower Limb & $820-829$ & 35 & 4 & 0 & 0 \\
\hline -Sprains \& Strains - Back & $846-847$ & 180 & 0 & 0 & 0 \\
\hline -Sprains \& Strains - Other & $840-845,848$ & 0 & 0 & 0 & 166 \\
\hline -Open Wound - Upper Limb & 880-887 & 96 & 0 & 0 & 0 \\
\hline -Open Wound - Lower Limb & $890-897$ & 2 & 0 & 0 & 0 \\
\hline -Contusion & $920-924$ & 55 & 0 & 0 & 0 \\
\hline -Burns & $940-949$ & 0 & 0 & 0 & 0 \\
\hline
\end{tabular}

*OSHA events with >1 ICD-9-CM code in the same diagnostic category were counted only once. Only those diagnostic categories and gender/job category combinations with at least one occurrence appear in this table. 
East Tennessee Technology Park 2007

OSHA Data

Appendix U. Number of Occurrences in Each Accident Category by Gender and Job Category*

\begin{tabular}{|c|c|c|c|}
\hline & & \multicolumn{2}{|c|}{ Women } \\
\hline & & Job Category & \multirow[b]{2}{*}{ TOTAL } \\
\hline & & Service & \\
\hline Type of Accident & E CODES & \multirow[b]{2}{*}{1} & \multirow[b]{2}{*}{1} \\
\hline Falls & E880-E888 & & \\
\hline Other Accidents & E916-E928 & 3 & 3 \\
\hline
\end{tabular}

\begin{tabular}{|c|c|c|c|c|c|}
\hline & \multicolumn{4}{|c|}{ Men } \\
\hline & & \multicolumn{3}{|c|}{ Job Category } & \multirow[b]{2}{*}{ TOTAL } \\
\hline & & Service & $\begin{array}{l}\text { Security and } \\
\text { Fire }\end{array}$ & $\begin{array}{c}\text { Line } \\
\text { Operators }\end{array}$ & \\
\hline Type of Accident & E CODES & \multirow[b]{2}{*}{1} & \multirow[b]{2}{*}{0} & \multirow[b]{2}{*}{0} & \multirow[b]{2}{*}{1} \\
\hline Falls & E880-E888 & & & & \\
\hline Other Accidents & E916-E928 & 3 & 1 & 1 & 5 \\
\hline
\end{tabular}

*Only those accident types and gender/job category combinations with at least one occurrence appear in this table. 
East Tennessee Technology Park 2007

OSHA Data

Appendix V. Number of Workdays Lost or with Restricted Activity in Each Accident Category by Gender and Job Category*

\begin{tabular}{|c|c|c|c|}
\hline & \multicolumn{2}{|c|}{ Women } \\
\hline & & \multicolumn{2}{|c|}{ Job Category } \\
\hline & & \multicolumn{2}{|c|}{ Service } \\
\hline & & $\begin{array}{c}\text { Days } \\
\text { Restricted }\end{array}$ & Days Lost \\
\hline Type of Accident & E Codes & \multirow[b]{2}{*}{0} & \multirow[b]{2}{*}{0} \\
\hline Falls & E880-E888 & & \\
\hline Other Accidents & E916-E928 & 94 & 0 \\
\hline
\end{tabular}

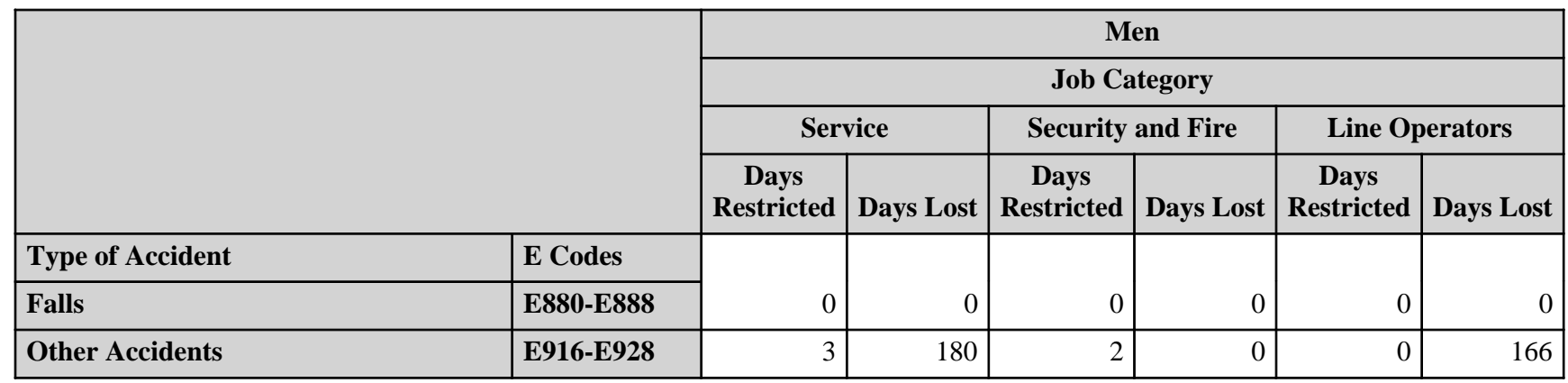

*OSHA events with $>1 \mathrm{E}$ code in the same accident type were counted only once. Only those accident types and gender/job category combinations with at least one occurrence appear in this table. 
East Tennessee Technology Park 2007

OSHA Data

Appendix W. Age-Adjusted OSHA Illness and Injury Rates by Diagnostic Category*

Part 1. Men

\begin{tabular}{|c|c|c|c|c|c|}
\hline & & $\begin{array}{l}\text { Number of } \\
\text { Diagnoses }\end{array}$ & $\begin{array}{c}\text { Age-Adjusted } \\
\text { Rate per } 1,000 * *\end{array}$ & $\begin{array}{c}\text { Lower } 95 \% \\
\text { Confidence } \\
\text { Limit per } 1,000\end{array}$ & $\begin{array}{c}\text { Upper } 95 \% \\
\text { Confidence } \\
\text { Limit per 1,000 }\end{array}$ \\
\hline Diagnostic Category & ICD-9-CM Code & \multirow[b]{2}{*}{2} & \multirow[b]{2}{*}{1.0} & \multirow[b]{2}{*}{0.2} & \multirow[b]{2}{*}{4.1} \\
\hline NERVOUS SYSTEM (NS) \& SENSE ORGANS & 320-389 & & & & \\
\hline -Disorders of Peripheral NS & $350-359$ & 2 & 1.0 & 0.2 & 4.1 \\
\hline INJURY \& POISONING & $800-999$ & 21 & 14.8 & 9.4 & 23.5 \\
\hline -Fracture - Upper Limb & 810-819 & 3 & 2.3 & 0.7 & 7.3 \\
\hline -Fracture - Lower Limb & $820-829$ & 1 & 0.6 & 0.1 & 4.5 \\
\hline -Sprains \& Strains - Back & \begin{tabular}{|l|}
$846-847$ \\
\end{tabular} & 3 & 2.5 & 0.7 & 8.5 \\
\hline -Sprains \& Strains - Other & $840-845,848$ & 5 & 2.3 & 0.9 & 5.7 \\
\hline -Open Wound - Upper Limb & 880-887 & 4 & 3.4 & 1.2 & 9.4 \\
\hline -Open Wound - Lower Limb & $890-897$ & 1 & 1.0 & 0.1 & 7.2 \\
\hline -Contusion & $920-924$ & 3 & 1.6 & 0.5 & 5.1 \\
\hline -Burns & $940-949$ & 1 & 1.1 & 0.2 & 8.1 \\
\hline Total & & 23 & 15.8 & 10.2 & 24.6 \\
\hline
\end{tabular}

*Only those diagnostic categories with at least one occurrence appear in this table.

**Standardized to age distribution of 2000 U.S. population. 
East Tennessee Technology Park 2007

OSHA Data

Appendix W. Age-Adjusted OSHA Illness and Injury Rates by Diagnostic Category*

Part 2. Women

\begin{tabular}{|c|c|c|c|c|c|}
\hline & & $\begin{array}{l}\text { Number of } \\
\text { Diagnoses }\end{array}$ & $\begin{array}{c}\text { Age-Adjusted } \\
\text { Rate per } 1,000^{* *}\end{array}$ & $\begin{array}{c}\text { Lower } 95 \% \\
\text { Confidence } \\
\text { Limit per } 1,000\end{array}$ & $\begin{array}{c}\text { Upper 95\% } \\
\text { Confidence } \\
\text { Limit per 1,000 }\end{array}$ \\
\hline Diagnostic Category & ICD-9-CM Code & \multirow[b]{2}{*}{6} & \multirow[b]{2}{*}{21.5} & \multirow[b]{2}{*}{7.9} & \multirow[b]{2}{*}{58.2} \\
\hline INJURY \& POISONING & 800-999 & & & & \\
\hline -Sprains \& Strains - Other & $840-845,848$ & 4 & 13.1 & 3.9 & 44.1 \\
\hline -Open Wound - Upper Limb & 880-887 & 1 & 1.1 & 0.2 & 7.8 \\
\hline -Open Wound - Lower Limb & $890-897$ & 1 & 7.3 & 1.0 & 51.5 \\
\hline Total & & 6 & 21.5 & 7.9 & 58.2 \\
\hline
\end{tabular}

*Only those diagnostic categories with at least one occurrence appear in this table.

**Standardized to age distribution of 2000 U.S. population. 
East Tennessee Technology Park 2007

OSHA Data

Appendix W. Age-Adjusted OSHA Illness and Injury Rates by Diagnostic Category*

Part 3. Men and Women

\begin{tabular}{|c|c|c|c|c|c|}
\hline & & $\begin{array}{l}\text { Number of } \\
\text { Diagnoses }\end{array}$ & $\begin{array}{c}\text { Age-Adjusted } \\
\text { Rate per } 1,000 * *\end{array}$ & $\begin{array}{c}\text { Lower } 95 \% \\
\text { Confidence } \\
\text { Limit per 1,000 }\end{array}$ & $\begin{array}{c}\text { Upper } 95 \% \\
\text { Confidence } \\
\text { Limit per 1,000 }\end{array}$ \\
\hline Diagnostic Category & ICD-9-CM Code & \multirow[b]{2}{*}{2} & \multirow[b]{2}{*}{0.7} & \multirow[b]{2}{*}{0.2} & \multirow[b]{2}{*}{2.9} \\
\hline NERVOUS SYSTEM (NS) \& SENSE ORGANS & 320-389 & & & & \\
\hline -Disorders of Peripheral NS & $350-359$ & 2 & 0.7 & 0.2 & 2.9 \\
\hline INJURY \& POISONING & 800-999 & 27 & 15.0 & 9.9 & 22.7 \\
\hline -Fracture - Upper Limb & $810-819$ & 3 & 1.6 & 0.5 & 5.3 \\
\hline -Fracture - Lower Limb & $820-829$ & 1 & 0.4 & 0.1 & 3.1 \\
\hline -Sprains \& Strains - Back & 846-847 & 3 & 2.0 & 0.6 & 6.9 \\
\hline -Sprains \& Strains - Other & $840-845,848$ & 9 & 4.2 & 2.1 & 8.8 \\
\hline -Open Wound - Upper Limb & $880-887$ & 5 & 2.9 & 1.1 & 7.5 \\
\hline -Open Wound - Lower Limb & 890-897 & 2 & 1.7 & 0.4 & 7.0 \\
\hline -Contusion & $920-924$ & 3 & 1.1 & 0.4 & 3.6 \\
\hline -Burns & $940-949$ & 1 & 1.0 & 0.1 & 6.9 \\
\hline Total & & 29 & 15.7 & 10.5 & 23.4 \\
\hline
\end{tabular}

*Only those diagnostic categories with at least one occurrence appear in this table.

**Standardized to age distribution of 2000 U.S. population. 